\title{
A METAMATERIAL-BASED MULTIBAND PHASE SHIFTER
}

\author{
A Dissertation \\ Submitted to the Graduate Faculty \\ of the \\ North Dakota State University \\ of Agriculture and Applied Science \\ By \\ Michael Maassel \\ In Partial Fulfillment of the Requirements \\ for the Degree of \\ DOCTOR OF PHILOSOPHY \\ Major Department: \\ Electrical and Computer Engineering
}

October 2013

Fargo, North Dakota 


\section{North Dakota State University \\ Graduate School}

Title

A Metamaterial-Based Multiband Phase Shifter

By

Michael Maassel

The Supervisory Committee certifies that this disquisition complies with

North Dakota State University's regulations and meets the accepted standards

for the degree of

\section{DOCTOR OF PHILOSOPHY}

\section{SUPERVISORY COMMITTEE:}

David Rogers

Co-Chair

Benjamin Braaten

Co-Chair

Subbaraya Yuvarajan

Orven Swenson

Robert Nelson

Approved:

January 9, 2014

Date
Scott Smith

Department Chair 


\begin{abstract}
A design methodology for a multi-band phase shifter using a metamaterial-based transmission line was developed. This method is different in that the loaded-line phase shifter has a phase shift of $90^{\circ}$ at the center frequencies of both bands instead of $-90^{\circ}$ and $-270^{\circ}$. The method was validated using simulation and measured results.
\end{abstract}




\section{ACKNOWLEDGMENTS}

I am very grateful to take this opportunity to thank Dr. Benjamin D. Braaten, Dr. David A. Rogers, Dr. Subbaraya Yuvarajan, Dr. Robert Nelson, and Dr. Orven F. Swenson for serving on my graduate committee. I would like to thank Dr. David A. Rogers and Dr. Benjamin D. Braaten for their willingness to be my co-advisors. 


\section{DEDICATION}

To my wife Karen and my children Patrick, Megan, and Benjamin. 


\section{TABLE OF CONTENTS}

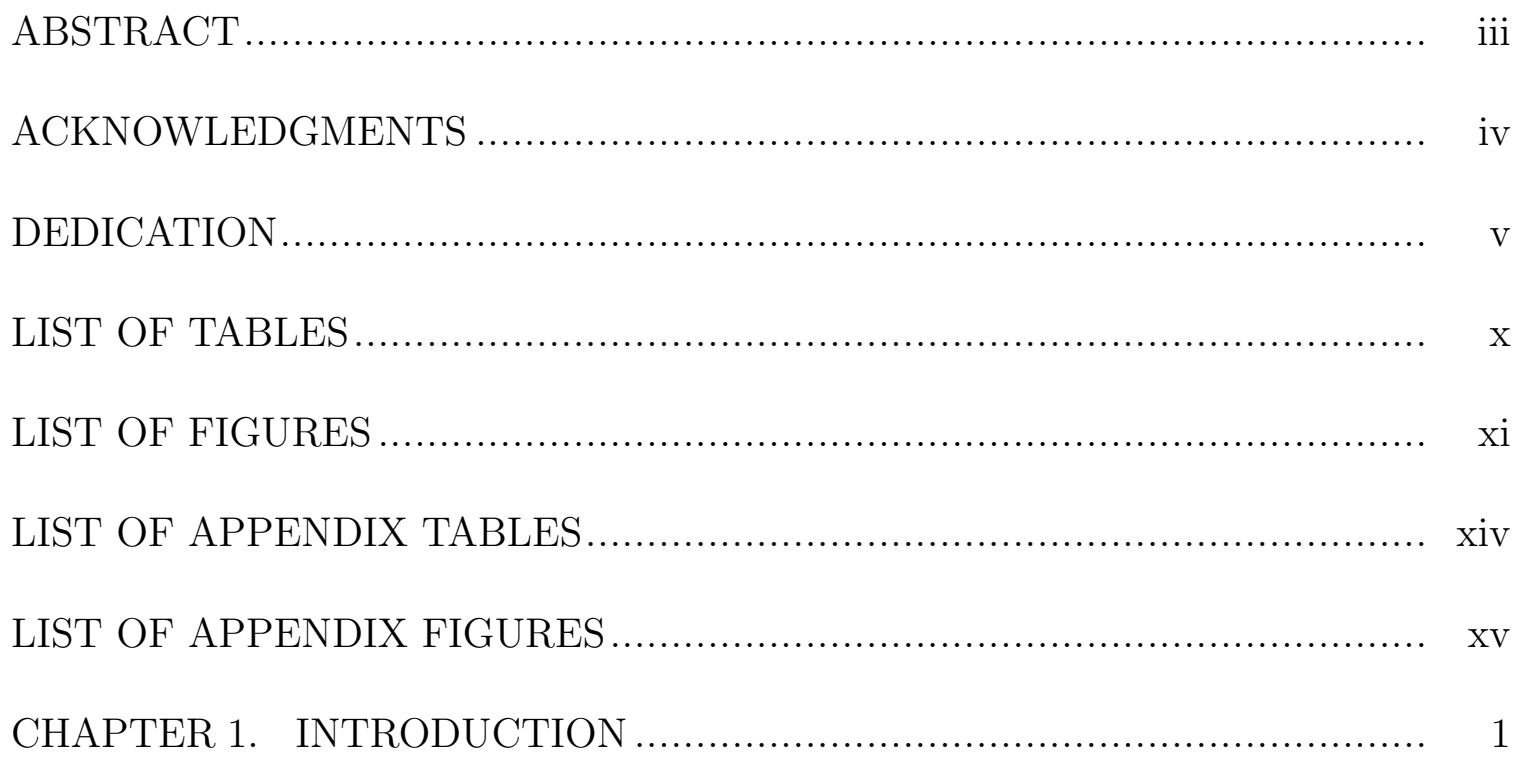

1.1. An Introduction to Metamaterials ........................ 1

1.2. Metamaterial-Based or Left-Handed Transmission Lines .. 1

1.2.1. Previous Work ...................................... 2

1.3. Metamaterial Transmission Line Design ...................... 3

1.3.1. A Review of the Analysis ............................ 3

1.4. Infinite Periodic Structure with Loads that are in Series and Parallel ................................................. 9

1.5. Bloch Impedance Derivation ............................. 11

1.6. Propagation Constant and Phase Velocity Derivations ..... 14

1.6.1. Right-Hand Transmission Line ...................... 14

1.6.2. Left-Hand Transmission Line........................ 15

1.7. Dispersion............................................... 15 
1.8. Calculation of the Series Capacitor and Shunt Inductor Values

CHAPTER 2. PHASE SHIFTERS …................................................... 19

2.1. Introduction .................................................... 19

2.1.1. Previous Work on Phase Shifters ...................... 20

2.1.2. Previous Work on Metamaterial-Based Phase Shifters ................................................... 20

2.2. Phase Shifter Parameters ....................................... 20

2.3. Types of Phase Shifters .......................................... 21

2.3.1. Switched Line Phase Shifter ............................ 21

2.3.2. Loaded Line Phase Shifter ............................... 22

2.3.3. Reflection Phase Shifter ................................ 22

2.4. Phase Shifter Selection ….................................... 22

2.5. Design Procedure …............................................... 23

2.5.1. Proposed Research ....................................... 27

CHAPTER 3. A NEW ANALYTIC DESIGN PROCEDURE FOR A METAMATERIAL PHASE SHIFTER .............................. 29

CHAPTER 4. SIMULATION ................................................................ 35

4.1. Ideal Components............................................... 35

4.2. Real Components .............................................. 36

4.3. Complete Phase Shifter........................................... 39

4.3.1. Infinitesimal Conditions ................................. 41

CHAPTER 5. FABRICATION …....................................................... 43 
5.1. Component Size Selection ....................................... 43

5.1.1. Capacitor and Inductor Footprints .................... 43

5.2. Layout of the Printed Circuit Board ............................ 45

5.2.1. Transmission Line Steps and Tapers ................... 45

5.2.2. Tapers …............................................... 45

5.2.3. Steps …................................................... 46

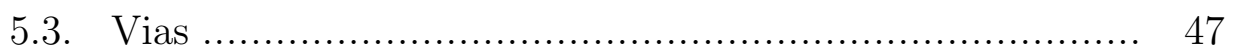

5.4. Thermal Reliefs .................................................. 48

5.4.1. Momentum Evaluation of a Thermal Relief .......... 50

5.5. Bias Networks ........................................................ 55

5.6. Fabrication ….................................................. 56

CHAPTER 6. TESTING ….......................................................... 58

6.1. Equipment Used .............................................. 58

6.2. Testing and Results .......................................... 58

6.3. Overall Performance ................................................... 64

6.3.1. Phase Shift Range .......................................... 64

6.3.2. Phase Shift Increment................................... 64

6.3.3. Frequency and Bandwidth of Operation.............. 64

6.3.4. Physical Size ................................................. 64

6.3.5. Insertion Loss.............................................. 64

6.3.6. Return Loss (or Impedance Matching) ................ 65

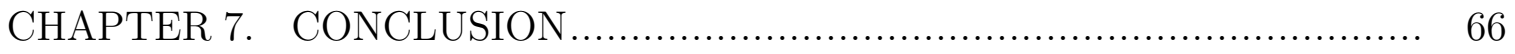


APPENDIX A. DERIVATION FOR THE ABCD MATRIX FOR THE D/2 TRANSMISSION LINE ................................... 70

APPENDIX B. ABCD MATRIX SIMPLIFICATION FOR THE PERIODICALLY LOADED INFINITE TRANSMISSION LINE.

APPENDIX C. ANALYSIS OF A RECIPROCAL NETWORK .................. 73

APPENDIX D. TRUNCATION ERROR FOR COS $\theta$.......................... 74

APPENDIX E. CASE 1: $\alpha=0, \beta \neq 0$ AND CASE 2: $\alpha \neq 0, \beta=0$ DERIVATIONS ................................................ 75

APPENDIX F. BLOCH IMPEDANCE DERIVATION .......................... 76

APPENDIX G. ABCD MATRIX FOR A LOSSLESS LOADED-LINE PHASE SHIFTER ........................................ 78

APPENDIX H. DERIVING THE MAGNITUDE AND ANGLE OF $S_{21}$ FOR A LOADED LINE PHASE SHIFTER......................... 80

APPENDIX I. CALCULATION OF THE DESIRED GOALS FOR THE INSERTION LOSS AND RETURN LOSS .................... 82

I.1. Desired Insertion Loss................................. 82

I.2. Desired Return Loss ..................................... 82

I.3. $920 \mathrm{MHz}$ Insertion Loss and Return Loss Analysis......... 83

I.4. $2450 \mathrm{MHz}$ Insertion Loss and Return Loss Analysis ........ 83

APPENDIX J. ANALYSIS OF THE MURATA ELECTRONICS CAPACITOR AND INDUCTOR MODELS USED IN THE ADS SIMULATION 


\section{LIST OF TABLES}

$\underline{\text { Table }}$

Page

1. Data for Linecalc for a Microstrip Transmission Line. ...................... 32

2. Data for Linecalc for a Coplanar Waveguide with Ground (CPWG)....... 33

3. Physical Parameters for the Transmission Lines............................ 33

4. $\quad$ Capacitance Values for Different Phase Shifts at $915 \mathrm{MHz} \ldots \ldots \ldots \ldots \ldots \ldots \ldots . . . . . . .34$

5. Capacitance Values for Different Phase Shifts at $2450 \mathrm{MHz} . \ldots \ldots \ldots \ldots \ldots \ldots . . . . . . .34$

6. Phase and Magnitude Readings for the $920 \mathrm{MHz}$ and $2450 \mathrm{MHz}$ Outputs. 36

7. Phase and Magnitude Readings for the $920 \mathrm{MHz}$ and $2450 \mathrm{MHz}$

Outputs. ................................................................... 38

8. Phase Shift, Insertion, and Return Loss for values of Reverse Bias Voltages. 40

9. Phase Shift, Insertion, and Return Loss for values of Reverse Bias Voltages. 41

10. Surface Mount Capacitor Footprint Dimensions.............................. 43

11. Surface Mount Inductor Footprint Dimensions............................ 44

12. Printed Circuit Board Material Specifications............................ 44

13. $S_{11}$ and Impedance for Different Lengths of Thermal Reliefs............... 55

14. Phase Shift for each Bias Voltage with a 1-pF Series Capacitor............. 60

15. Insertion and Return Loss for each Bias Voltage with a 1-pF Series

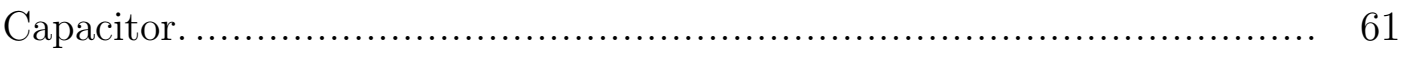

16. Phase Shift for each Bias Voltage with a 10-pF Series Capacitor............ 62

17. Insertion and Return Loss for each Bias Voltage with a 10-pF Series Capacitor. .................................................................... 63 


\section{LIST OF FIGURES}

Figure

$\underline{\text { Page }}$

1. Electromagnetic Bandgap Metamaterial. ......................................... 2

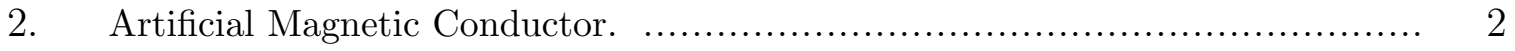

3. $\quad$ Infinitely Long Periodically Loaded Transmission Line. ......................... 4

4. Generalized Infinite Periodic Transmission Line with Series and Parallel Loads. 10

5. $\quad$ Dispersion Diagram for a Right-Handed Transmission Line. . ................... 16

6. Dispersion Diagram for a Left-Handed Transmission Line. ...................... 17

7. Lumped Element Representation of a Composite Transmission Line Unit Cell. . 17

8. Microstrip Implementation of a Composite Transmission Line Unit Cell........ 18

9. Right-Handed Lumped-Element Transmission Line Unit Cell. .............. 18

10. Left-Handed Lumped-Element Transmission Line Unit Cell. $\quad \ldots \ldots \ldots \ldots \ldots \ldots \ldots \ldots \ldots \ldots$

11. Switched-Line Phase Switcher. ............................................. 21

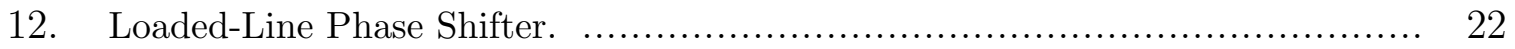

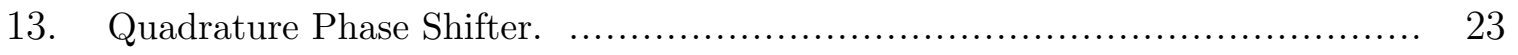

14. Proposed Metamaterial-Based Phase Shifter. ................................. 28

15. ADS Phase Shifter - Ideal Components with Exact Values. ................. 35

16. $920 \mathrm{MHz}$ Output: Phase of $S_{21}$, Magnitude of $S_{21}$, and Magnitude of $S_{11}$. ... 36

17. $2450 \mathrm{MHz}$ Output: Phase of $S_{21}$, Magnitude of $S_{21}$, and Magnitude of $S_{11} \ldots \quad 36$

18. $\quad$ ADS Phase Shifter - Murata Components. .................................. 38

19. $920 \mathrm{MHz}$ Output: Phase of $S_{21}$, Magnitude of $S_{21}$, and Magnitude of $S_{11}$. ... 38

20. $2450 \mathrm{MHz}$ Output: Phase of $S_{21}$, Magnitude of $S_{21}$, and Magnitude of $S_{11} \ldots \ldots$ 
21. Simulation Schematic with Murata Components and S-Parameter Files for the Varactor Diodes. ......................................................... 39

22. The Capacitance versus Reverse Bias Voltage for the Varactor Diode. .......... 40

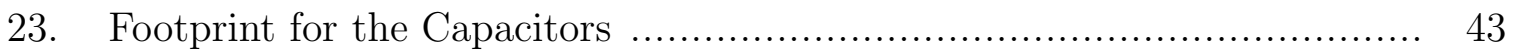

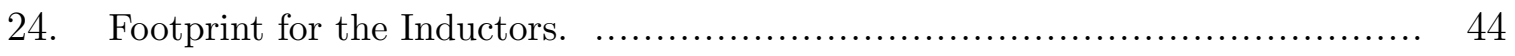

25. Simulation Schematic for the Taper from the CPWG to an SMA Connector. .. 45

26. Simulation Output for the Taper from the CPWG to an SMA Connector. ..... 46

27. Simulation Schematic of a Microstrip Step to an 0402 Component. ............. 46

28. Simulation Output of a Microstrip Step to an 0402 Component. ............... 47

29. Simulation Schematic of the Different Via Models and the Reference Circuits. 48

30. Simulation Output of the Different Via Models and the Reference Circuits. ... 49

31. Tombstoned Passive Component. ......................................... 49

32. Thermal Relief. .......................................................... 50

33. Momentum Layout without Thermal Reliefs. .............................. 51

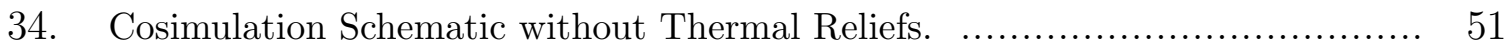

35. Cosimulation Output without Thermal Reliefs. .............................. 52

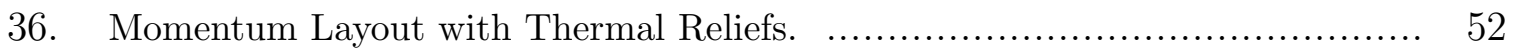

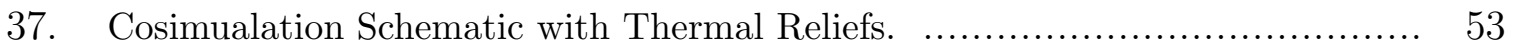

38. Cosimualation Output with Thermal Reliefs. .............................. 53

39. Simulation Schematic for Adjusting the Lengths of the Thermal Reliefs. ....... 54

40. Simulation Output with Different Lengths of Thermal Reliefs. ................. 54

41. DC Bias Network Schematic .......................................... 55 
42. Insertion Loss of the DC Bias Network.

43. Final Layout for the Printed Circuit Board. .................................. 56

44. Assembled Printed Circuit Board. ......................................... 57

45. Close Up of the Varactor Diode and Bias Network. ............................. 57

46. Magnitude of $S_{11}$ and $S_{21}$ of the Phase Shifter with the DC Bias for the Varactor Diodes Set at 1 Volt.

47. Phase Shift and Magnitude of $S_{21}$ of the Phase Shifter with the DC Bias for the Varactor Diodes Set at 1 Volt. 


\section{LIST OF APPENDIX TABLES}

$\underline{\text { Table }}$

$\underline{\text { Page }}$

D.1. Error versus Number of Terms........................................... 74

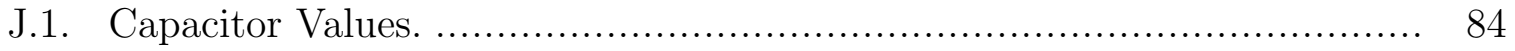

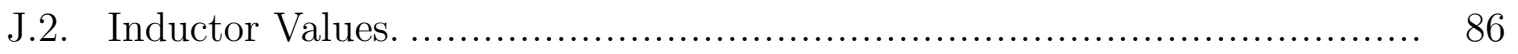




\section{LIST OF APPENDIX FIGURES}

Figure

J.1. Comparison between a Murata Electronics Capacitor and Ideal ADS Component Capacitor Model. ................................................

J.2. Comparison between a Murata Electronics Capacitor and Ideal ADS Component Capacitor Model. .................................................

J.3. Comparison between a Murata Electronics Inductor and Ideal ADS Component Inductor Model...............................................

J.4. Comparison between a Murata Electronics Inductor and Ideal ADS Component Inductor Model.................................................... 


\section{CHAPTER 1. INTRODUCTION}

Phase shifters are used in several different microwave and RF applications, including phased array antennas, radar systems, and phase noise measurement systems. The current research on metamaterial-based phase shifters is very limited. The research relies on simulations to provide the values for the metamaterial portion of the phase shifter, a method that can be prone to errors. The methodolgy developed here provides analytically computed values for the metamaterial transmission line before the circuit is simulated. These values were validated with both simulation and measured results.

An interesting aspect of this research is that there is very limited information about the electrical design and electrical performance of thermal reliefs at higher frequencies. To explore this aspect further, simulations were performed using a circuit simulator and a 2.5-D electromagnetic simulator to determine the electrical performance of the thermal reliefs.

\subsection{An Introduction to Metamaterials}

Metamaterials are artificial media with properties not readily available in nature. Examples of metamaterials include electromagnetic band gaps, artificial magnetic conductors, and double-negative or left handed materials [1]. Electromagnetic band gap structures have frequency bands (band gaps) where electromagnetic waves cannot propagate as shown in Figure 1 [1]. Artificial magnetic conductors have a normal reflection phase of $0^{\circ}$ at the resonant frequency as shown in Figure 2 [1]. Double-negative or left handed materials have both a negative permittivity and permeability, giving a negative phase velocity.

\subsection{Metamaterial-Based or Left-Handed Transmission Lines}

The metamaterial structure that is used in this work is the left-handed (LH) transmission line. The advantage of a left-handed transmission line is that the phase 


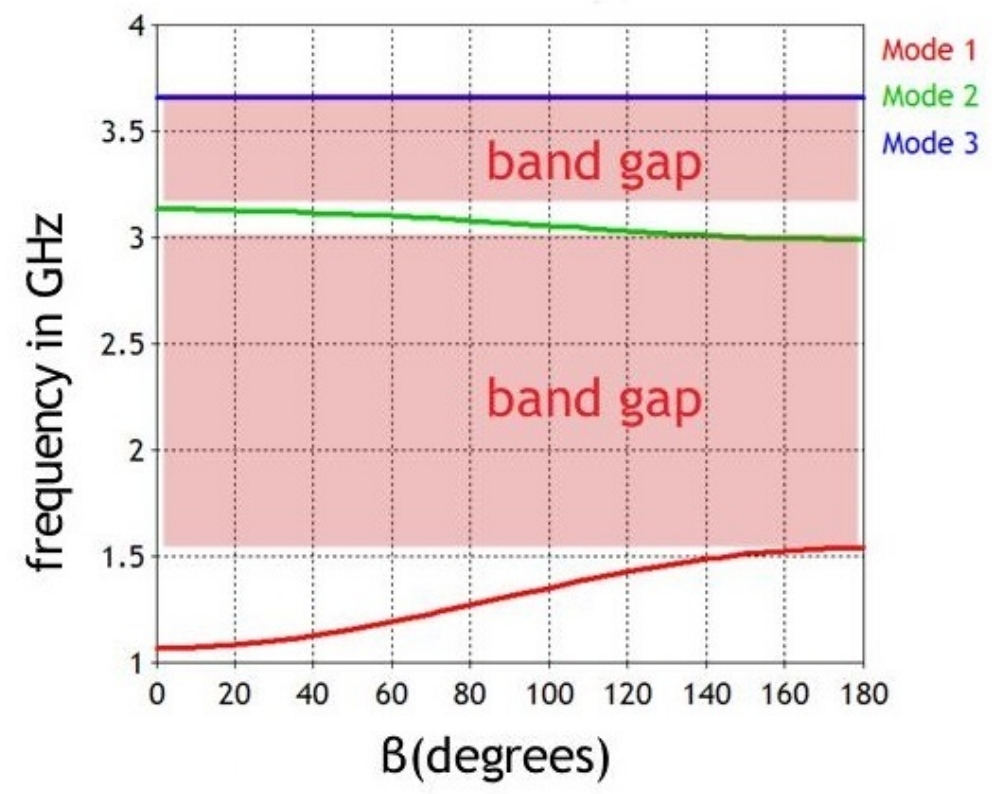

Figure 1. Electromagnetic Bandgap Metamaterial.

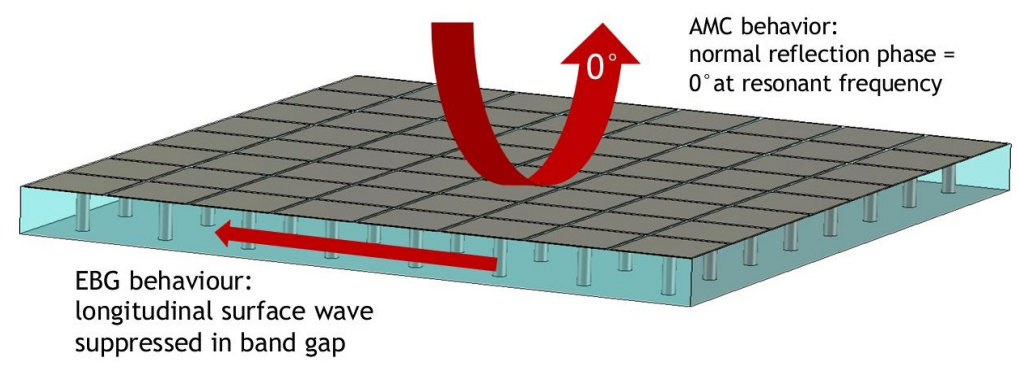

Figure 2. Artificial Magnetic Conductor.

shift is negative as the signal moves down the transmission line. As shown in (1.1) and (1.2) the exponential term of the left-handed transmission line is positive compared to negative as for a right-handed $(\mathrm{RH})$ transmission line. However, the flow of energy is still the same as defined by the Poynting vector. The negative phase shift of the left-handed transmission line was verified by plotting (with Matlab) the phase versus distance of both (1.1) and (1.2).

\subsubsection{Previous Work}

Caloz and Itoh [2] applied the theory of left-handed transmission line to the realization of a microstrip based left-handed transmission lines. Braaten and Scheeler 
[3] derived the equations for the Bloch impedance, propagation constants and phase velocity for the infinite periodically loaded structure and the infinite periodic structure with loads that are in series and parallel.

$$
v(z)=V^{+} e^{-j \beta z}
$$

and

$$
v(z)=V^{+} e^{j \beta z}
$$

Where

$$
\begin{aligned}
& \mathrm{v}(\mathrm{z})=\text { the voltage along the transmission line } \\
& \mathrm{V}+=\text { the incident voltage on the transmission line } \\
& \beta=\text { the propagation constant of the transmission line } \\
& \mathrm{z}=\text { the position along the transmission line }
\end{aligned}
$$

\subsection{Metamaterial Transmission Line Design}

\subsubsection{A Review of the Analysis}

When analyzing any type of transmission line, one needs to obtain expressions for the propagation constant, phase velocity, and the impedance. Figure 3 shows the equivalent circuit for an infinitely long transmission line that is periodically loaded. The voltage and current on either side of the $n^{\text {th }}$ unit cell is expressed with the following ABCD matrices:

$$
\left[\begin{array}{l}
V_{n} \\
I_{n}
\end{array}\right]=\left[\begin{array}{ll}
A & B \\
C & D
\end{array}\right]\left[\begin{array}{l}
V_{n+1} \\
I_{n+1}
\end{array}\right] .
$$




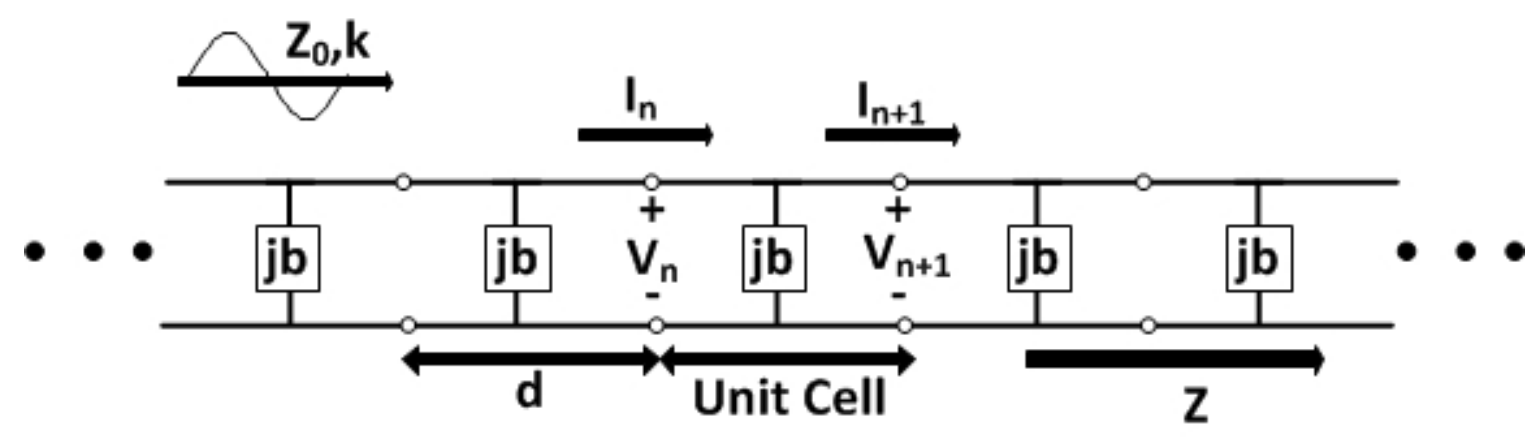

Figure 3. Infinitely Long Periodically Loaded Transmission Line.

The ABCD matrix of the unit cell is composed of three distinct ABCD matrices [4]:

$$
\begin{aligned}
\text { transmission line of length } \mathrm{d} / 2 & \Rightarrow\left[\begin{array}{cc}
\cos \frac{\theta}{2} & j Z_{0} \sin \frac{\theta}{2} \\
j Y_{0} \sin \frac{\theta}{2} & \cos \frac{\theta}{2}
\end{array}\right], \\
\text { shunt element } & \Rightarrow\left[\begin{array}{cc}
1 & 0 \\
j b & 1
\end{array}\right],
\end{aligned}
$$

and

$$
\text { transmission line of length } \mathrm{d} / 2 \Rightarrow\left[\begin{array}{cc}
\cos \frac{\theta}{2} & j Z_{0} \sin \frac{\theta}{2} \\
j Y_{0} \sin \frac{\theta}{2} & \cos \frac{\theta}{2}
\end{array}\right] \text {. }
$$

In (1.2) through (1.4), $\theta=\beta d$ where $\beta$ is the propagation constant of the transmission line and $\mathrm{d}$ is the length of the unit cell. Appendix A gives the details of how the ABCD matrix was derived for the $\mathrm{d} / 2$ transmission line. When the matrices are normalized to $Z_{0}$, (1.2) through (1.4) reduce to the following:

$$
\begin{gathered}
\text { transmission line of length } \mathrm{d} / 2 \Rightarrow\left[\begin{array}{cc}
\cos \frac{\theta}{2} & j \sin \frac{\theta}{2} \\
j \sin \frac{\theta}{2} & \cos \frac{\theta}{2}
\end{array}\right], \\
\text { shunt element } \Rightarrow\left[\begin{array}{cc}
1 & 0 \\
j b & 1
\end{array}\right],
\end{gathered}
$$


and

$$
\text { transmission line of length } \mathrm{d} / 2 \Rightarrow\left[\begin{array}{cc}
\cos \frac{\theta}{2} & j \sin \frac{\theta}{2} \\
j \sin \frac{\theta}{2} & \cos \frac{\theta}{2}
\end{array}\right] \text {. }
$$

To determine the ABCD matrix for the infinitely long, periodically loaded transmission line, matricies (1.5) through (1.7) are multiplied together. This then gives

$$
\left[\begin{array}{ll}
A & B \\
C & D
\end{array}\right]=\left[\begin{array}{cc}
\cos \frac{\theta}{2} & j Z_{0} \sin \frac{\theta}{2} \\
j Y_{0} \sin \frac{\theta}{2} & \cos \frac{\theta}{2}
\end{array}\right]\left[\begin{array}{cc}
1 & 0 \\
j b & 1
\end{array}\right]\left[\begin{array}{cc}
\cos \frac{\theta}{2} & j Z_{0} \sin \frac{\theta}{2} \\
j Y_{0} \sin \frac{\theta}{2} & \cos \frac{\theta}{2}
\end{array}\right],
$$

which results in the complete ABCD matrix for the infinitely long, periodically loaded transmission line (refer to Appendix B):

$$
\left[\begin{array}{ll}
A & B \\
C & D
\end{array}\right]=\left[\begin{array}{cc}
\left(\cos \theta-\frac{b}{2}\right) & j\left(\sin \theta+\frac{b}{2} \cos \theta-\frac{b}{2}\right) \\
j\left(\sin \theta+\frac{b}{2} \cos \theta+\frac{b}{2}\right. & \left(\cos \theta-\frac{b}{2} \sin \theta\right)
\end{array}\right] .
$$

For a wave propagating in the $+\mathrm{z}$ direction, the voltage and current can be written as

$$
V(z)=V(0) e^{-\gamma z}
$$

and

$$
I(z)=I(0) e^{-\gamma z}
$$

respectively. Since the transmission line is infinitely long, the $\mathrm{n}^{\text {th }}$ terminal voltage and current and the subsequent terminal voltage and current differ only by the propagation factor. This then gives the following for the $n+1$ terminal voltage and currents:

$$
V_{n+1}=V_{n} e^{-\gamma z}
$$


and

$$
I_{n+1}=I_{n} e^{-\gamma z}
$$

This can be written in terms of an ABCD matrix as

$$
\left[\begin{array}{c}
V_{n} \\
I_{n}
\end{array}\right]=\left[\begin{array}{ll}
A & B \\
C & D
\end{array}\right]\left[\begin{array}{l}
V_{n+1} \\
I_{n+1}
\end{array}\right]=\left[\begin{array}{c}
V_{n+1} e^{\gamma d} \\
I_{n+1} e^{\gamma d}
\end{array}\right] .
$$

Subtracting the right side matrix from both sides of (1.14) yields:

$$
\left[\begin{array}{ll}
A & B \\
C & D
\end{array}\right]\left[\begin{array}{l}
V_{n+1} \\
I_{n+1}
\end{array}\right]-\left[\begin{array}{l}
V_{n+1} e^{\gamma d} \\
I_{n+1} e^{\gamma d}
\end{array}\right]=0
$$

and

$$
\left[\begin{array}{cc}
A-e^{\gamma d} & B \\
C & D-e^{\gamma d}
\end{array}\right]\left[\begin{array}{c}
V_{n+1} \\
I_{n+1}
\end{array}\right]=0 .
$$

The determinant of the above matrix must equal zero if the solution is nontrivial [5]:

$$
A D+e^{2 \gamma d}-(A+D) e^{\gamma d}-B C=0 .
$$

For a reciprocal device: $\mathrm{AD}-\mathrm{BC}=1$. This is derived in Appendix $\mathrm{C}$. This then gives

$$
1+e^{2 \gamma d}-(A+D) e^{\gamma d}=0
$$

This yields

$$
e^{\gamma d}+e^{-\gamma d}=A+D
$$

Using the definition of the hyperbolic cosine,

$$
\cosh \gamma d=\frac{e^{\gamma d}+e^{-\gamma d}}{2}
$$


Substituting the values for A and D (from 1.11) yields

$$
\cosh \gamma d=\frac{A+D}{2}=\cos \theta-\frac{b}{2} \sin \theta
$$

and

$$
\cosh \alpha d \cos \beta d+j \sinh \alpha d \sin \beta d=\cos \theta-\frac{b}{2} \sin \theta
$$

. Now, the right-hand side of (1.23) is always a real number, therefore either $\alpha$ or $\beta$ must be equal to 0 . For illustration, consider the following cases, which are based on the derivations provided in Appendix E.

Case 1: $\alpha=0$ and $\beta \neq 0$

This is the non-attenuating propagating wave and it defines the pass-band of the transmission line. Thus

$$
\cos \beta d=\cos \theta-\frac{b}{2} \sin \theta
$$

If the right side of 1.25 is less then or equal to $1, \beta$ can be determined. There can be an infinite number of values for $\beta$.

Case 2: $\alpha \neq 0$ and $\beta=0$

This is a non-propagating attenuating wave, so it defines the stop-band of the transmission line. Since the line is lossless, power is not dissipated, but is reflected back to the input. Thus,

$$
\cosh \alpha d=\left|\cos \theta-\frac{b}{2} \sin \theta\right| \geq 1 \text {. }
$$

If $\cos \theta-\frac{b}{2} \sin \theta \leq-1,(1.26)$ can be obtained from (1.24) by letting $\beta=\pi$. Then all of the lumped loads are $\frac{\lambda}{2}$ apart. This yields an input impedance the same as if $\beta=0$. 
Therefore the periodically loaded line will have either pass-bands or stop-bands, depending on the frequency and the normalized susceptance loads. This will then permit the structure to be viewed as a type of filter.

Now that an expression for the propagation constant has been determined, the Bloch impedance cell will be determined. The Bloch impedance is defined as the characteristic impedance of the waves on the structure [6]. The Bloch impedance is given by

$$
Z_{B}=Z_{0} \frac{V_{n+1}}{I_{n+1}}
$$

From (1.18),

$$
\left(A-e^{\gamma d}\right) V_{n+1}+B I_{n+1}=0
$$

and

$$
\frac{V_{n+1}}{I_{n+1}}=\frac{-B}{A-e^{\gamma d}}
$$

Therefore

$$
Z_{B}=\frac{-Z_{0} B}{A-e^{\gamma d}}
$$

From (1.19) (repeated here for ease of reading), an expression for $e^{\gamma d}$ is determined to be:

$$
e^{2 \gamma d}-(A+D) e^{\gamma d}+1=0
$$

The Bloch impedance is then

$$
Z_{B}^{ \pm}=\frac{-2 Z_{0} B}{2 A-(A+D) \mp \sqrt{(A+D)^{2}-4}}
$$

or

$$
=\frac{-2 Z_{0} B}{A-D \mp \sqrt{(A+D)^{2}-4}} .
$$


Since the cell is symmetric, $\mathrm{A}=\mathrm{D}$ and

$$
Z_{B}^{ \pm}=\frac{ \pm Z_{0} B}{\sqrt{A^{2}-1}}
$$

$Z_{B}^{+}$defines a positively traveling wave, and $Z_{B}^{-}$is for a negatively traveling wave.

There are two cases to consider here.

Case 1: $\alpha=0$ and $\beta \neq 0$

From (1.23) and since the network is symmetrical $(\mathrm{A}=\mathrm{D})$,

$$
\cosh \gamma d=\frac{A+D}{2}=\frac{A+A}{2}=A
$$

The magnitude of A will always be less than or equal to 1 (refer to Appendix E for the analysis). Since B is always imaginary (refer to 1.11) then the Bloch impedance is real since the denominator is also imaginary.

Case 2: $\alpha \neq 0$ and $\beta=0$

For Case 2, A will always be greater than or equal to 1 (refer to Appendix E for the analysis). Then the Bloch impedance will be imaginary.

\subsection{Infinite Periodic Structure with Loads that are in Series and Parallel}

Transmission lines (both RH and LH) can be modeled with series and parallel lumped components. The next step is to derive the impedance and propagation constant of this transmission line model.

In Figure 4, the unit cell is composed of a series element, a shunt element, and another series element. The ABCD parameters are [4]:

$$
\text { Series Element } \Rightarrow\left[\begin{array}{cc}
1 & Z_{S} \\
0 & 1
\end{array}\right] \text {, }
$$




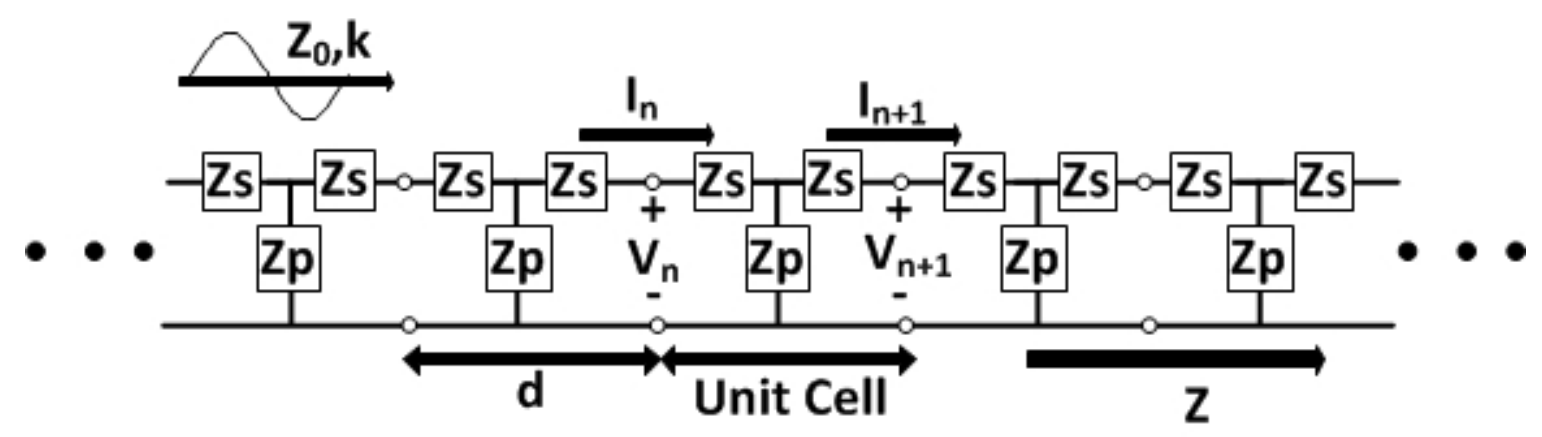

Figure 4. Generalized Infinite Periodic Transmission Line with Series and Parallel Loads.

$$
\text { Shunt Element } \Rightarrow\left[\begin{array}{cc}
1 & 0 \\
\frac{1}{Z_{P}} & 1
\end{array}\right] \text {, }
$$

and

$$
\text { Series Element } \Rightarrow\left[\begin{array}{cc}
1 & Z_{S} \\
0 & 1
\end{array}\right] .
$$

Now the three ABCD matrices are combined to give the final ABCD matrix for the unit cell:

$$
\left[\begin{array}{cc}
A & B \\
C & D
\end{array}\right]=\left[\begin{array}{cc}
1 & Z_{S} \\
0 & 1
\end{array}\right]\left[\begin{array}{cc}
1 & 0 \\
\frac{1}{Z_{P}} & 1
\end{array}\right]\left[\begin{array}{cc}
1 & Z_{S} \\
0 & 1
\end{array}\right],
$$

so that

$$
\left[\begin{array}{ll}
A & B \\
C & D
\end{array}\right]=\left[\begin{array}{cc}
1+\frac{Z_{s}}{Z_{p}} & Z_{s} \\
\frac{1}{Z_{p}} & 1
\end{array}\right]\left[\begin{array}{cc}
1 & Z_{S} \\
0 & 1
\end{array}\right],
$$

and, finally,

$$
\left[\begin{array}{ll}
A & B \\
C & D
\end{array}\right]=\left[\begin{array}{cc}
1+\frac{Z_{s}}{Z_{p}} & 2 Z_{s}+\frac{Z_{s}^{2}}{Z_{P}} \\
\frac{1}{Z_{p}} & 1+\frac{Z_{s}}{Z_{p}}
\end{array}\right] .
$$

Now that the ABCD matrix for the unit cell has been derived, the impedance and propagation constant will be determined. The analysis is very similar to the infinitely long, periodically loaded transmission line analyzed previously. The 
determinate of (1.41) must equal 0 if the solution is nontrivial [5], implying

$$
A D+e^{2 \gamma d}-(A+D) e^{\gamma d}-B C=0
$$

For a reciprocal device: $\mathrm{AD}-\mathrm{BC}=1$

$$
\begin{gathered}
1+e^{2 \gamma d}-(A+D) e^{\gamma d}=0 \\
A+D=e^{\gamma d}+e^{-\gamma d}
\end{gathered}
$$

Substitute the values for A and D from (1.41) and using the definition of the hyperbolic cosine (1.22) yields:

$$
\cosh \gamma d=\frac{A+D}{2}=1+\frac{Z_{s}}{Z_{p}} .
$$

Since

$$
\gamma=\alpha+j \beta
$$

then

$$
\cosh [(\alpha+j \beta) d]=1+\frac{Z_{s}}{Z_{p}} .
$$

\subsection{Bloch Impedance Derivation}

Case $1(\alpha=0$ and $\beta \neq 0)$

Using the trignometric identity $[\cosh (j \beta d)=\cos (\beta d)$,$] equation (1.47) gives the$ following:

$$
\cos (\beta d)=1+\frac{Z_{s}}{Z_{p}}
$$


The Bloch impedance is given by:

$$
Z_{B}=\frac{-Z_{0} B}{A-e^{\gamma d}}
$$

Solving for $e^{\gamma d}$ from (1.43) gives

$$
e^{\gamma d}=\frac{(A+D) \pm \sqrt{(A+D)^{2}-4}}{2}
$$

Solving for $Z_{B}$ yields (refer to Appendix $\mathrm{F}$ for the complete derivation) the following:

$$
Z_{B}=-Z_{0} \sqrt{Z_{s}\left(2 Z_{p}+Z_{s}\right)} .
$$

The next step is to define the Bloch impedance in terms of the series and parallel elements that were used to define the transmission line. For the right-handed (RH) transmission line, the series elements are inductors.

$$
Z_{s}=\frac{j \omega L}{2} .
$$

Note: the inductor value is divided by 2 since there are two identical inductors in the unit cell. The shunt elements are capacitors with

$$
Z_{p}=\frac{1}{j \omega C} .
$$

Rewriting (1.48) (where the subscript $\mathrm{R}$ is used to denote the $\mathrm{RH}$ transmission line) yields:

$$
\cos \beta_{R} d=1+\frac{\frac{j \omega L}{2}}{\frac{1}{j \omega C}}=1+\frac{-\omega^{2} L C}{2}
$$


Equation (1.49) is rewritten as

$$
\begin{aligned}
& Z_{B R}=-Z_{0} \sqrt{\frac{j \omega L}{2}\left(2 \frac{1}{j \omega C}+\frac{j \omega L}{2}\right)}=-Z_{0} \sqrt{\frac{L}{C}-\frac{\omega^{2} L^{2}}{4}}=-Z_{0} \sqrt{\frac{4 L-\omega^{2} L C}{4 C}} \\
& =-Z_{0} \sqrt{\frac{4 L}{4 C}-\frac{\omega^{2} L^{2} C}{4 C}}=-Z_{0} \sqrt{\frac{L}{C}-\frac{L}{C} \frac{\omega^{2}}{\omega_{C R}^{2}}}=-Z_{0} \sqrt{\frac{L}{C}\left(1-\frac{\omega^{2}}{\omega_{C R}^{2}}\right)}
\end{aligned}
$$

where

$$
\omega_{C R}^{2}=\left(\frac{2}{\sqrt{L C}}\right)^{2} .
$$

For the left-handed (LH) transmission line the, series element is a capacitor with

$$
Z_{s}=\frac{1}{j 2 \omega C} .
$$

Again the 2 in the denominator occurs since there are two capacitors in the unit cell. The shunt element is an inductor with

$$
Z_{P}=j \omega L .
$$

Therefore

$$
\cos \left(\beta_{L} d\right)=1-\frac{1}{2 \omega^{2} L C}
$$

and

$$
Z_{B L}=-Z_{0} \sqrt{\frac{1}{j 2 \omega C}\left(j 2 \omega L+\frac{1}{j 2 \omega C}\right)}=-Z_{0} \sqrt{\frac{L}{C}-\frac{1}{4 \omega^{2} C^{2}}}=-Z_{0} \sqrt{\frac{L}{C}\left(1-\frac{\omega_{C L}^{2}}{\omega^{2}}\right)}
$$

where

$$
\omega_{C L}^{2}=\left(\frac{2}{\sqrt{L C}}\right)^{2} .
$$




\subsection{Propagation Constant and Phase Velocity Derivations}

The propagation constant and phase velocity derivations for both $\mathrm{RH}$ and $\mathrm{LH}$ transmission lines are presented in this section.

\subsubsection{Right-Hand Transmission Line}

The starting point will be (1.54) (repeated here for ease of reading) which is

$$
\cos \beta_{R} d=1+\frac{-\omega^{2} L C}{2}
$$

Next, (1.63) is rewritten using a MacLaurin Series expansion in the following manner:

$$
\cos \left(\beta_{R} d\right) \approx \cos (0)+\beta_{R} d \sin (0)-\frac{\left(\beta_{R} d\right)^{2}}{2 !} \cos (0)
$$

The truncation error for terminating this series after the third element is less

then $10 \%$ (see Appendix D for the truncation error analysis). Substituting (1.64) into (1.63) yields:

$$
\cos \beta_{R} d \approx 1-\frac{\left(\beta_{R} d\right)^{2}}{2 !}=1-\frac{-\omega^{2} L C}{2}
$$

Solving for $\beta_{R} d$ gives

$$
\beta_{R} d=\omega \sqrt{L C}
$$

Then the phase velocity is

$$
V_{\phi R}=\frac{\omega}{\beta_{R}}=\frac{d}{\sqrt{L C}}>0
$$

and the group velocity is

$$
V_{g R}=\left(\frac{\partial \beta_{R}}{\partial \omega}\right)^{-1}=V_{\phi R}>0
$$




\subsubsection{Left-Hand Transmission Line}

Begin with (1.60) (repeated here for ease of reading) which is

$$
\cos \beta_{L} d=1-\frac{1}{2 \omega^{2} L C}
$$

The process is identical to the right-hand transmission line where

$$
\cos \beta_{L} d \approx 1-\frac{\left(\beta_{L} d\right)^{2}}{2 !}=1-\frac{1}{2 \omega^{2} L C}
$$

The phase constant is given by

$$
\beta_{L} d=\frac{-1}{\omega \sqrt{L C}}
$$

and the phase velocity is

$$
V_{\phi L}=\frac{\omega}{\beta_{L}}=-\omega^{2} d \sqrt{L C}<0 .
$$

Finally, the group velocity is the following:

$$
V_{g L}=\left(\frac{\partial \beta_{L}}{\partial \omega}\right)^{-1}=-V_{\phi L}>0
$$

\subsection{Dispersion}

An important part of any wideband structure is its dispersion. Dispersion occurs since waves at different frequencies will have different velocities. This can lead to deformed waveforms at the end of the structure. The propagation constants for the RH and LH transmission lines are

$$
\beta_{R} d=\omega \sqrt{L C}>0
$$


and

$$
\beta_{L} d=\frac{-1}{\omega \sqrt{L C}}<0
$$

Now solving for $\omega$ in each equation gives

$$
\omega=\frac{\beta_{R} d}{\sqrt{L C}}
$$

and

$$
\omega=\frac{-1}{\beta_{L} d \sqrt{L C}}
$$

Equations (1.76) and (1.77) are plotted in the Figures 5 and 6.

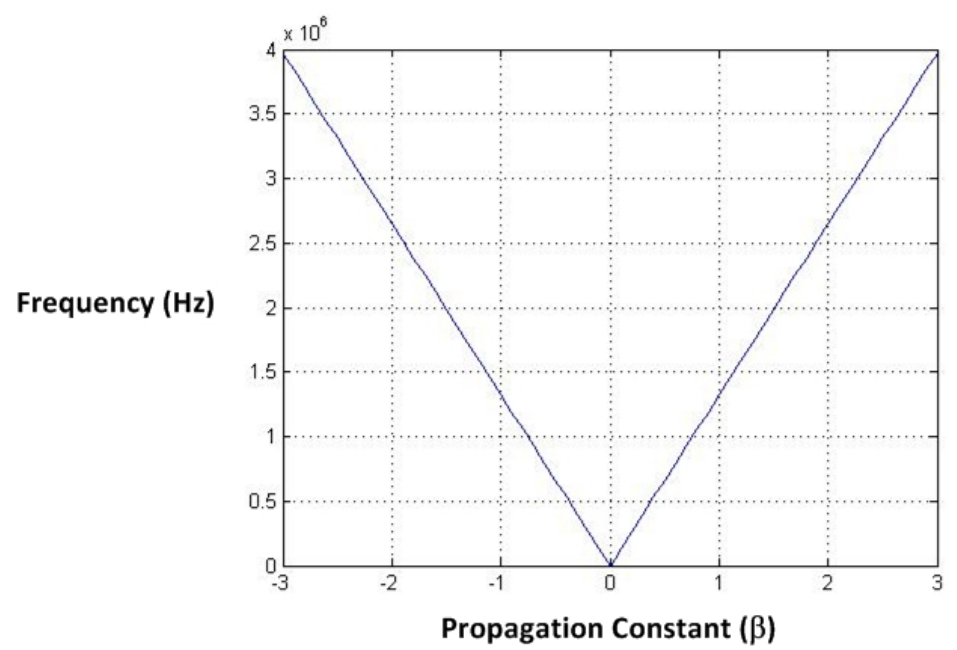

Figure 5. Dispersion Diagram for a Right-Handed Transmission Line.

Now that the equations for the propagation constant and impedance have been determined for the lumped-element transmission line, the design of the LH transmission line can begin. A left-handed transmission line can be created by adding series capacitance and shunt inductance to a right-handed transmission line as shown in Figure 7. This is referred to as a combination right-left-handed transmission line (CRLH). The series capacitance can be added by using an inter-digital capacitor. The shunt inductance is added by using grounded stubs as shown in Figure 8. The stub that creates $L_{\text {Left }}$ is between $\frac{\lambda}{10}$ and $\frac{\lambda}{4}$ in length. If multiple stubs are needed they typically have a spacing of $\frac{\lambda}{10}$. 


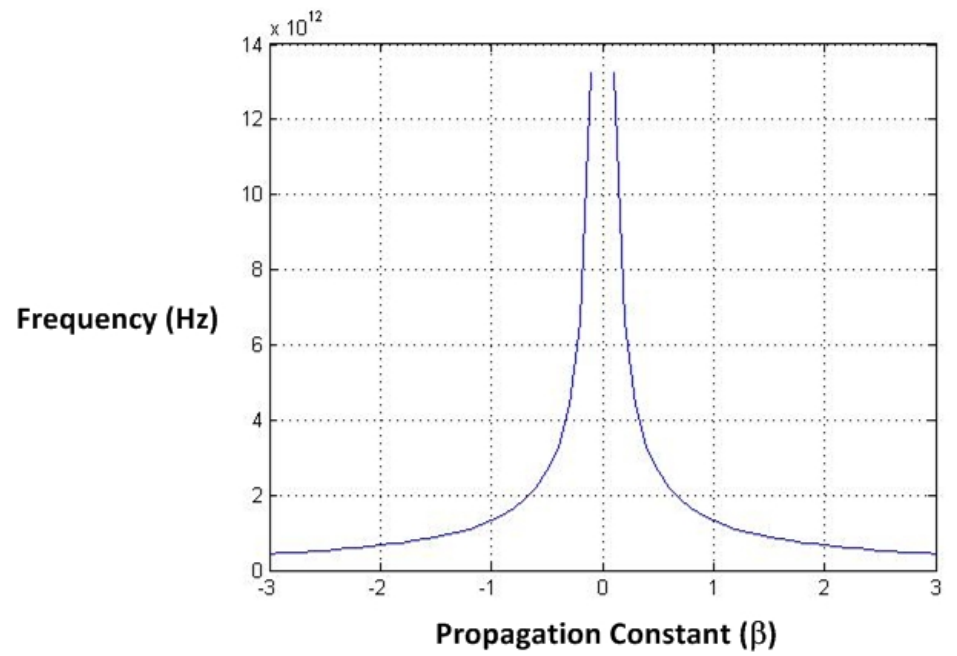

Figure 6. Dispersion Diagram for a Left-Handed Transmission Line.

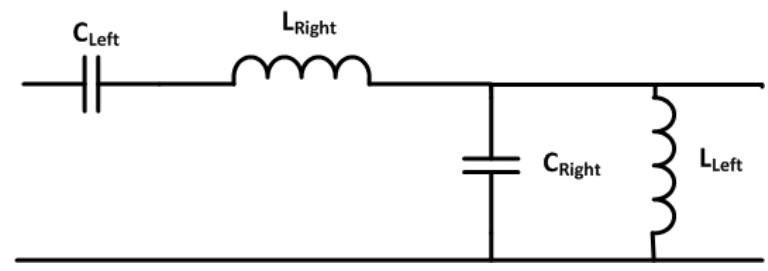

Figure 7. Lumped Element Representation of a Composite Transmission Line Unit Cell.

\subsection{Calculation of the Series Capacitor and Shunt Inductor Values}

At low frequencies, the CRLH is predominately LH and at high frequencies it is $\mathrm{RH}$. To insure that there are no discontinuites at the transition frequency (which is given by $\left.\omega_{C}=\sqrt[4]{L_{L} C_{L} L_{R} C_{R}}\right), \beta_{C}=\beta_{R}+\beta_{L}$ and $Z_{0 L}=Z_{0 R}[7]$. These two conditions are achieved by careful selection of the lumped element values that make up the CLRH transmission line. The unit cells of the transmission lines can be modeled with lumped elements (see Figures 9 and 10). 


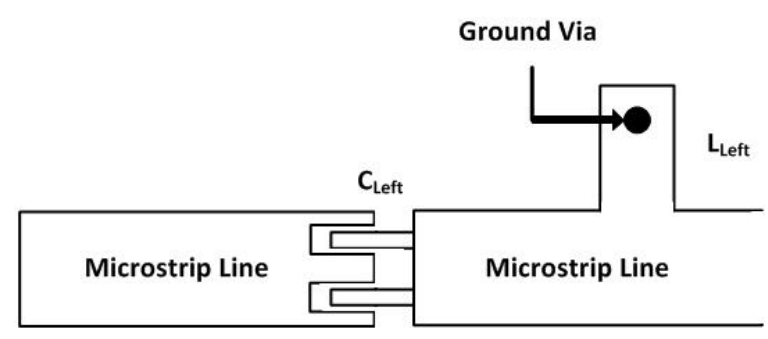

Figure 8. Microstrip Implementation of a Composite Transmission Line Unit Cell.

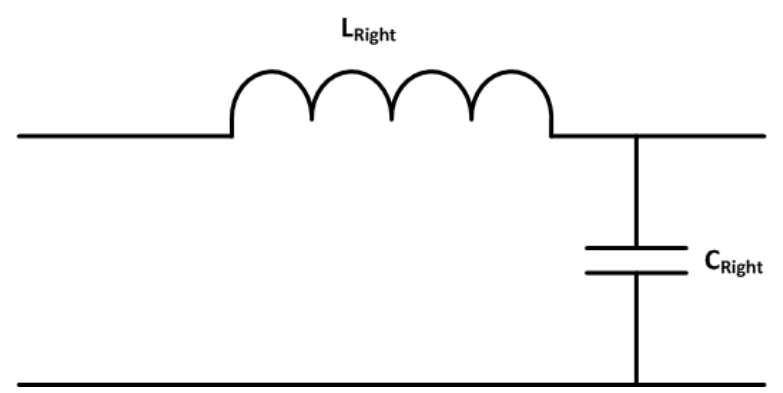

Figure 9. Right-Handed Lumped-Element Transmission Line Unit Cell.

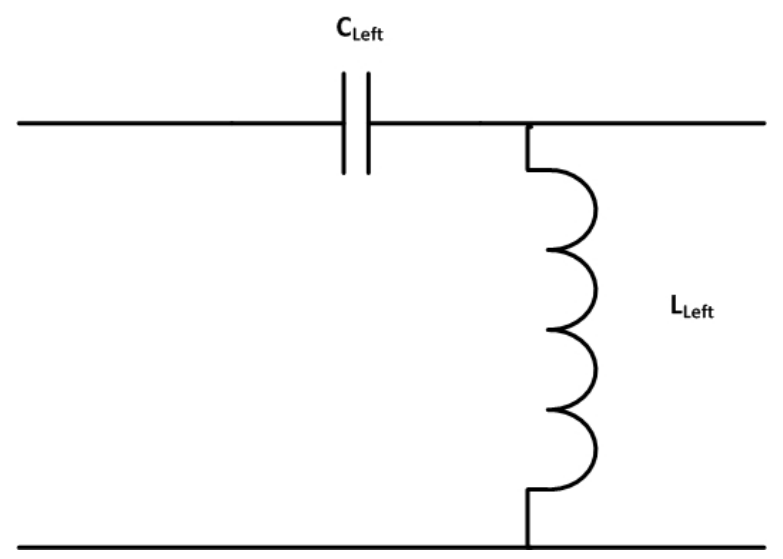

Figure 10. Left-Handed Lumped-Element Transmission Line Unit Cell. 


\section{CHAPTER 2. PHASE SHIFTERS}

\subsection{Introduction}

One of the main applications where phase shifters are used is in phased antenna arrays. The phase shifters are used to adjust the location of the main lobe of the array. This permits the beam to be steered electronically instead of mechanically. This area has been dominated by the military and aerospace industries due to the high cost of the arrays.

However, there are commercial applications that would benefit greatly from phased arrays. Typically these applications are high data rate communications, such as video conferencing, cruise ships, and airlines. Low earth orbit (LEO) satellites may provide the needed infrastructure. LEO systems have three main advantages over geostationary (GEO) satellites.

1. LEO orbits are typically less then $1000 \mathrm{~km}$ away instead of the $35,000 \mathrm{~km}$ for the GEO. This provides an automatic power savings of $30 \mathrm{~dB}$.

2. LEO satellites have a nearly imperceptible propagation delay. This is an extremely important aspect for high data rate communications.

3. There is the possibility of a significant reduction in the cost of launching the satellites.

Recently metamaterials (left-handed materials) have begun to appear in commercial products. Left-handed materials are defined by the direction of the wave vector $\mathrm{k}$. In left-handed materials the left hand is used to curl the electric field into the magnetic field, instead of the right hand for traditional materials (right-handed).

The advantage of using left-handed materials in the phase shifter is that the phase shift can be centered on $0^{\circ}$ as opposed to $\pm 180^{\circ}$ for a right-handed phase 
shifter. This is highly advantageous if there are several phase shifters in series. If multiple right-handed phase shifters were used, an increase in group delay, physical size and insertion loss will occur.

A reconfigurable phase shifter has the advantage that it can be used on several different frequency bands. This would simplify the circuit design and dramatically reduce the cost of the antenna array.

\subsubsection{Previous Work on Phase Shifters}

Several authors have analyzed the loaded-line phase shifter using ABCD matrices for example, Garver [8], White [9], and Yahara [10]. Atwate [11] derived the condition for symmetric switching about $90^{\circ}$. Opp and Hoffman [12] defined three classes of loading modes. Davis [13] developed equations to determine the operational bandwidth of a loaded-line phase shifter.

\subsubsection{Previous Work on Metamaterial-Based Phase Shifters}

Recent published research [14] through [18] does not present a comprehensive methodology for designing a metamaterial-based phase shifter, but relies on simulation and/or building the phase shifter and then adjusting the component values to obtain the desired results. While this is a valid technique, it is very time consuming and prone to errors.

\subsection{Phase Shifter Parameters}

Basically any variable reactance in series or shunt across a transmission line can be used to introduce a phase shift. The design parameters that need to be considered when designing a phase shifter are:

- Phase Shift Range

- Phase Shift Increment

- Frequency of Operation 
- Bandwidth of Operation

- Physical Size

- Insertion Loss

- Return Loss (or Impedance Matching)

- Analog or Digital Control

\subsection{Types of Phase Shifters}

There are two main categories of phase shifters: ferrite and semiconductor. This work will concentrate on semiconductor phase shifters. Semiconductor phase shifters are divided into different categories based on how they produce the phase shift. The basic categories are: switched line, loaded line, and reflection.

\subsubsection{Switched Line Phase Shifter}

One of the simplest phase shifters is the switched line (Figure 11). The delay arm is physically longer; hence, it introduces a phase shift. The number of positions on the switch can be increased to include additional phase shifts.

While this is a very simple and useful phase shifter, it is limited to a narrow bandwidth of operation. Also this phase shifter is limited to discrete shifts; for example, $22.5^{\circ}, 45^{\circ}, 90^{\circ}$, and $180^{\circ}$. If this frequency of operation is low, this phase shifter can become very large.

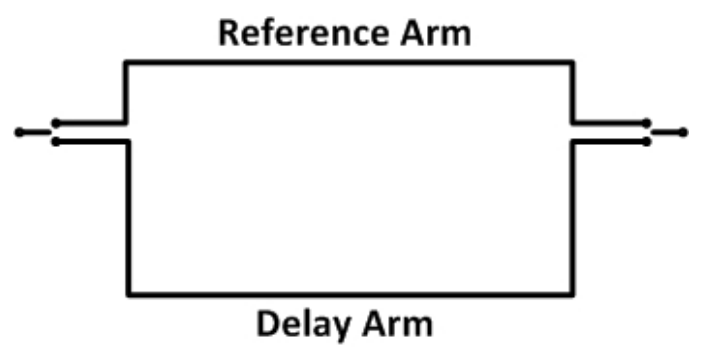

Figure 11. Switched-Line Phase Switcher. 
An important design parameter of the switched-line phase shifter is the isolation between the two lines. Typically the isolation must be $20 \mathrm{~dB}$ or greater. If the isolation is less than $20 \mathrm{~dB}$ there will be ripples in the amplitude and phase response. The switches can be PIN diodes, FETs or MEMs. Electomechanical switches (relays) are usually not used because of their slow switching speed and contact bounce.

\subsubsection{Loaded Line Phase Shifter}

The loads must be highly reflective and are typically capacitors or inductors. The phase response of the loaded line (Figure 12) is usually flatter then the switchedline phase shifter. The bandwidth of the loaded-line phase shifter is limited because of the quarter-wavelength transmission line.

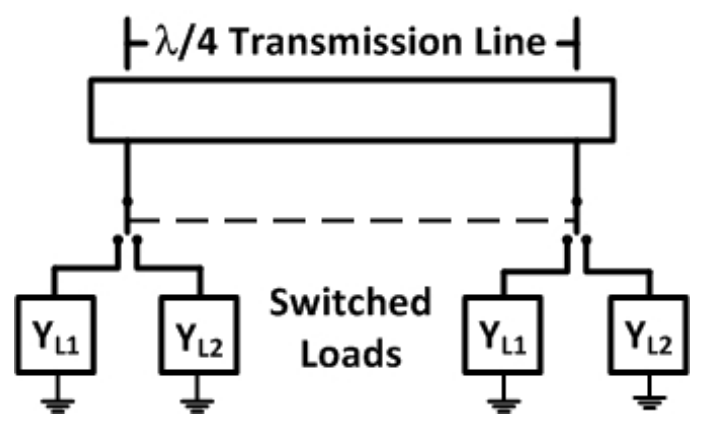

Figure 12. Loaded-Line Phase Shifter.

\subsubsection{Reflection Phase Shifter}

A reflection phase shifter is based on a quadrature hybrid (Figure 13). As with the other phase shifters, this one has a limited bandwidth due to the hybrid. Also the physical size of the phase shifter will become very large at low frequencies.

\subsection{Phase Shifter Selection}

Since a current need for low-cost phase shifters is in the cellular telephone area which also means low frequency operation (900 MHz), the loaded line model (Figure $12)$ is the preferred choice. 


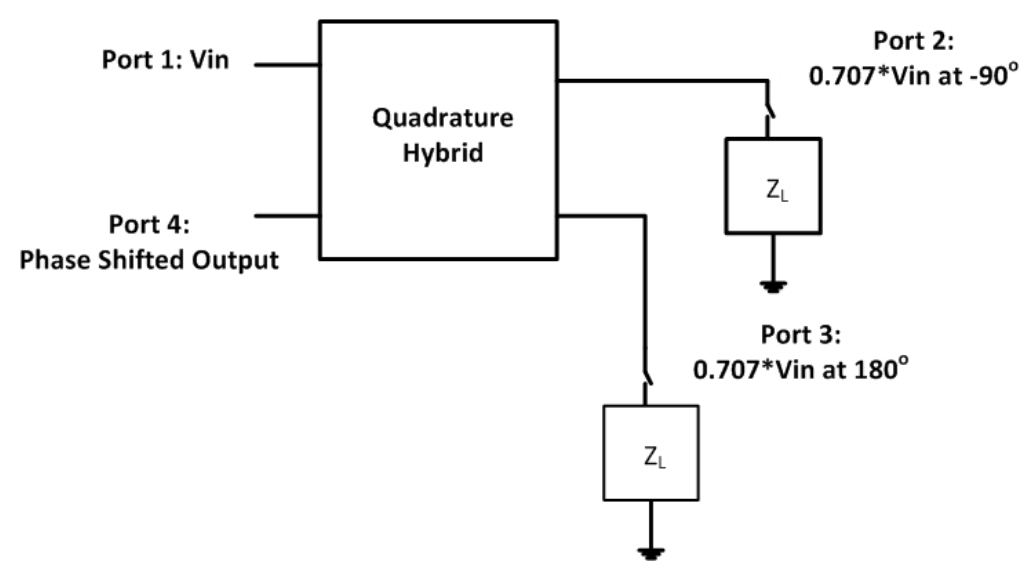

Figure 13. Quadrature Phase Shifter.

\subsection{Design Procedure}

The ABCD matrix of Figure 12 is equated to the ABCD matrix of a transmission line of electrical length $\theta$ and characteristic impedance of $Z_{C}$. Refer to Appendix $\mathrm{G}$ for the derivation of the ABCD matrix for the network.

$$
Y_{L 1}=G_{L 1}+B_{L 1}
$$

and

$$
Y_{L 2}=G_{L 2}+B_{L 2}
$$

For this derivation, the shunt loads will be lossless $\left(G_{L 1}=G_{L 2}=0\right)$ and $\theta=\beta L$. The phase shifter matrix is the product of three matricies:

$$
\left[\begin{array}{cc}
1 & 0 \\
j B_{L 1} & 1
\end{array}\right]\left[\begin{array}{cc}
\cos \beta l & j Z_{C} \sin \beta l \\
j Y_{C} \sin \beta l & \cos \beta l
\end{array}\right]\left[\begin{array}{cc}
1 & 0 \\
j B_{L 1} & 1
\end{array}\right]
$$

which simplifies to (refer to Appendix G for the complete derivation of (2.4))

$$
\left[\begin{array}{cc}
\cos \beta l-B_{L 1} Z_{C} \sin \beta l & j Z_{C} \sin \beta l \\
j Z_{C}\left(2 B_{L 1} Y_{C} \cos \beta l+\left(Y_{C}^{2}-B_{L 1}^{2}\right) \sin \beta l\right. & \cos \beta l-B_{L 1} Z_{C} \sin \beta l
\end{array}\right]
$$


The matrix elements are:

$$
\begin{gathered}
A=\cos \beta l-B_{L 1} Z_{C} \sin \beta l, \\
B=Z_{C} \sin \beta l \\
C=Z_{C}\left(2 B_{L 1} Y_{C} \cos \beta l+\left(Y_{C}^{2}-B_{L 1}^{2}\right) \sin \beta l,\right.
\end{gathered}
$$

and

$$
D=\cos \beta l-B_{L 1} Z_{C} \sin \beta l
$$

Network analyzers use S-parameters to measure RF or microwave circuits. Sparameters can be expressed in terms of ABCD parameters. $S_{11}$ is given by:

$$
S_{11}=\frac{B Y_{0}-C Z_{0}}{2 A+B Y_{0}+C Z_{0}} .
$$

$Z_{0}$ is defined as the characteristic impedance of the input and output of the phase shifter. Substituting (2.5) through (2.8) into (2.9) yields

$$
S_{11}=\frac{j Y_{0} Z_{C} \sin \beta l-j Z_{0} Z_{C}\left(2 B_{L 1} Y_{C} \cos \beta l+\left(Y_{C}^{2}-B_{L 1}^{2}\right) \sin \beta l\right.}{E+F+G}
$$

where

$$
\begin{gathered}
E=2\left(\cos \beta l-B_{L 1} Z_{C} \sin \beta l\right), \\
F=j Y_{0} Z_{C} \sin \beta l,
\end{gathered}
$$


and

$$
G=j Z_{0} Z_{C}\left(2 B_{L 1} Y_{C} \cos \beta l+\left(Y_{C}^{2}-B_{L 1}^{2}\right) \sin \beta l\right)
$$

It is important for the phase shifter to have a good match to the circuitry. In order for this to occur, $S_{11}$ should be zero (ideally). $S_{11}$ in terms of ABCD parameters is:

$$
S_{11}=\frac{A+\frac{B}{Z_{0}}-C Z_{0}-D}{A+\frac{B}{Z_{0}}+C Z_{0}-D} .
$$

Thus,

$$
S_{11}=0=A+\frac{B}{Z_{0}}-C Z_{0}-D=\frac{B}{Z_{0}}-C Z_{0}
$$

and

$$
\frac{B}{Z_{0}}=C Z_{0}
$$

Substituting the values for B and C from (2.6) and (2.7) into (2.16)

$$
\frac{j Z_{C} \sin \beta l}{Z_{0}}=j Z_{0}\left[Z_{C}\left(2 B_{L 1} Y_{C} \cos \beta l\right)+\left(Y_{C}^{2}-B_{L 1}^{2}\right) \sin \beta l\right],
$$

or

$$
Y_{0}^{2} Z_{C} \sin \beta l=Z_{C}\left(2 B_{L 1} Y_{C} \cos \beta l\right)+\left(Y_{C}^{2}-B_{L 1}^{2}\right) \sin \beta l
$$

and

$$
Y_{0}^{2}-Y_{C}^{2}+B_{L 1}^{2} \sin \beta l=2 B_{L 1}^{2} Y_{C}^{2} \cos \beta l
$$

With the matched input conditions $\left(B Y_{0}=C Z_{0}\right)$,

$$
S_{21}=\frac{2}{2 A+B Y_{0}+C Z_{0}}=\frac{1}{A+B Y_{0}} .
$$


Substituting (2.5) and (2.6) into (2.18) gives:

$$
S_{21}=\frac{1}{\cos \beta l-B_{L 1} Z_{C} \sin \beta l+j Z_{C} Y_{0} \sin \beta l} .
$$

Under matched conditions, $\left|S_{21}\right|=1$. Appendix $\mathrm{H}$ contains the complete derivation of the magnitude and angle of $S_{21}$. From Appendix H, (H.12) and (H.13) are repeated here:

$$
\angle S_{21}=\arccos \left(\cos \beta l-B_{L 1} Z_{C} \sin \beta l\right)
$$

and

$$
\angle S_{21}=\arcsin \left(-Z_{C} Y_{0} \sin \beta l\right)
$$

Equation (2.22) will assume two values from the two switching states $\left(B_{L 1}\right.$ and $\left.B_{L 2}\right)$, while $(2.23)$ remains unchanged. Both equations will be satisfied if the phase delay $(\phi)$ is symmetrically switched around $90^{\circ}$ in increments of $\frac{\Delta \phi}{2}$. Using $\phi=90^{\circ} \pm \frac{\Delta \phi}{2}$, analytic expressions for $Y_{C}$ and $B_{L}$ are derived as:

$$
\begin{gathered}
\sin \left(90^{\circ} \pm \frac{\Delta \phi}{2}\right)=\cos \frac{\Delta \phi}{2}=-Z_{C} Y_{0} \sin \beta l \\
Y_{C}=-Y_{0} \sec \left(\frac{\Delta \phi}{2}\right) \sin \beta l \\
\cos \left(90^{\circ} \pm \frac{\Delta \phi}{2}\right)=\cos \beta l-B_{L 1} Z_{C} \sin \beta l \\
\mp \sin \left(\frac{\Delta \phi}{2}\right)=\cos \beta l-B_{L 1} Z_{C} \sin \beta l,
\end{gathered}
$$

and

$$
B_{L 1}=-\frac{ \pm \sin \frac{\Delta \phi}{2}+\cos \beta l}{Z_{C} \sin \beta l}
$$


Substituting (2.25) into (2.28) then gives

$$
B_{L 1}=\left(\frac{-Y_{0} \sec \left(\frac{\Delta \phi}{2}\right) \sin (\beta l)}{\sin (\beta l)}\right)\left( \pm \sin \left(\frac{\Delta \phi}{2}\right)+\cos \beta l\right)
$$

and

$$
B_{L 1}=-Y_{0}\left[\cos (\beta l)\left[\sec \left(\frac{\Delta \phi}{2}\right)\right] \pm \tan \left(\frac{\Delta \phi}{2}\right)\right] .
$$

\subsubsection{Proposed Research}

The ideal phase shifter would have zero insertion loss and a variable phase shift from $0^{\circ}$ to $\pm 180^{\circ}$. While a low insertion loss $(<0.5 \mathrm{~dB})$ can be obtained by a careful design, there will always be a fixed phase shift due to the construction of the phase shifter. For example, the traditional loaded-line phase shifter will always have a phase shift around $90^{\circ}$ because of the $\frac{\lambda}{4}$ transmission line.

The configuration for the propsed metamaterial-based phase shifter is shown in Figure 14. The next step is to determine which conditions are required for the phase shifter operation. This will include the operating frequency ranges, phase shift values, insertion loss, input impedance, voltage standing wave ratio (VSWR), power handling capability, and phase shift error. It is necessary to define the operating conditions first since the selection of the varactor diodes directly affects several of these parameters. Once these parameters have been defined, the design methodology for the phase shifter will be developed. This will include using the equations for the metamaterial-based transmission line that were developed in chapter 1 . The next step will be to obtain the design parameters for the varactor diodes from the manufacturer or measurements using the network analyzer. Then the design equations will be developed for the phase-shifter.

Next, simulation models of the transmission line will be developed, along with models for the varactor diodes. Together, all of these models will be used to simulate 
the complete metamaterial-based phase shifter. This simulation will be performed using Agilent's Advanced Design System (ADS).

Once the simulation is working correctly, the printed circuit board will be fabricated and the circuit will be assembled. This circuit will then be tested at different frequencies and different phase shifts. A network analyzer will allow accurate and repeatable measurement of the insertion loss, phase shift, and input impedance of the circuit.

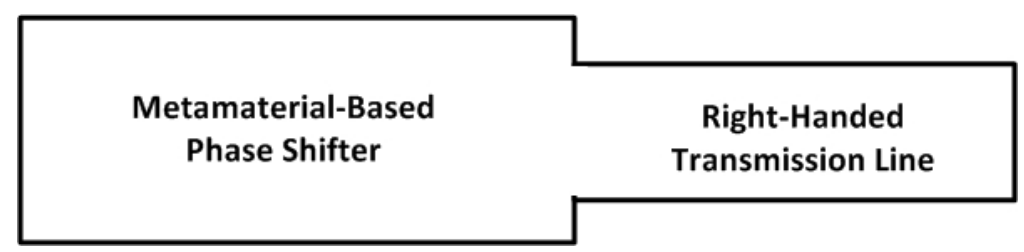

Figure 14. Proposed Metamaterial-Based Phase Shifter. 


\section{CHAPTER 3. A NEW ANALYTIC DESIGN PROCEDURE FOR A METAMATERIAL PHASE SHIFTER}

The loaded-line phase shifter was selected since it can have a broad range of phase shifts and the phase shifts are not confined to set values. The loaded-line phase shifter is a quarter-wavelength transmission line with shunt reactances at each end. The quarter-wavelength transmission line will be a composite right/left-handed (CRLH) transmission line. This will enable the phase shifter to provide both positive phase shifts and negative phase shifts as opposed to conventional phase shifters which can only provide positive or negative phase shifts.

The composite right/left-handed (CRLH) transmission line will be used for both of the desired frequency bands: $900 \mathrm{MHz}(902$ to $928 \mathrm{MHz})$ and $2400 \mathrm{MHz}(2400$ to $2498 \mathrm{MHz}$ ) ISM (Industrial, Scientific, and Medical) bands.

The method used for realizing a composite right/left-handed (CRLH) transmission line is with lumped elements. There are two areas of concern that need to addressed with a lumped-element CRLH transmission line unit cell:

1. The analysis of a CRLH line assumes that the unit cells are infinitely small; therefore, the physical size of each unit cell will be a factor.

2. The phase shift of each unit cell must be much less then $90^{\circ}$.

Since the phase shift of the entire structure will be $90^{\circ}$ and the transmission line is composed of several unit cells, the $90^{\circ}$ condition is not a major concern.

A practical approach is to ensure that the physical size of a lumped-element unit cell is less then $\frac{\lambda}{10}$ at the desired frequencies. The highest operating frequency will be $2500 \mathrm{MHz}$, which gives a wavelength as determined in (3.1):

$$
\lambda=\frac{3 \times 10^{8}}{2500 \times 10^{6}}=0.12 \text { meters. }
$$


Therefore the physical size of the lumped-element unit cell cannot be larger then a tenth of a wavelength as shown in (3.2):

$$
\frac{\lambda}{10}=\frac{0.12}{10}=0.012 \text { meters }=12 \mathrm{~mm}
$$

Lin et al. [19] gave a proceedure for designing a dual-band quarter-wavelength transmission line. However, the phase shifts are at $-90^{\circ}$ and $-270^{\circ}$ at the respective frequency bands. The desired phase shift is $90^{\circ}$ for both bands.

The desired phase shift at each frequency $f_{1}=915 \mathrm{MHz}$ and $f_{2}=2450 \mathrm{MHz}$ is

$$
\phi\left(f_{1}\right)=90^{\circ}
$$

and

$$
\phi\left(f_{2}\right)=90^{\circ}
$$

The total phase shift is equal to the sum of the phase shift from the right-handed and left-handed transmission lines, or

$$
\phi_{c}=\phi_{R}+\phi_{L}
$$

and

$$
\frac{\pi}{2}=-2 \pi f N \sqrt{L_{R} C_{R}}+\frac{N}{2 \pi f} \frac{1}{\sqrt{L_{L} C_{L}}}
$$

where $\mathrm{N}$ is the number of sections in the CRLH transmission line. Setting

$$
P=-2 \pi N \sqrt{L_{R} C_{R}}
$$

and

$$
Q=\frac{N}{2 \pi} \frac{1}{\sqrt{L_{L} C_{L}}},
$$


the inductor and capacitor values can be determined by the following steps:

Step 1: Solve for P and Q. Thus,

$$
P f_{1}+\frac{Q}{f_{1}}=90^{\circ}
$$

and

$$
P f_{2}+\frac{Q}{f_{2}}=90^{\circ}
$$

Solving these two equations for $\mathrm{P}$ and $\mathrm{Q}$ results in

$$
P=\frac{\pi}{2}\left(\frac{1}{f_{1}+f_{2}}\right)
$$

and

$$
Q=\frac{\pi}{2} f_{1} f_{2}\left(\frac{1}{f_{1}+f_{2}}\right)
$$

The values for $\mathrm{P}$ and $\mathrm{Q}$ are calculated, and the results are: $P=4.6680 \times 10^{-10}$ seconds and $Q=1.0465 \times 10^{9} \frac{1}{\text { seconds }}$.

Step 2: Choose a value for $\mathrm{N}$ in the equation. $\phi_{L}$ is the unit cell phase shift.

$$
\phi_{L}=N \phi_{L}
$$

$\mathrm{N}$ is arbitrarly choosen as 5; therefore,

$$
\phi_{L}=\frac{\phi_{L}}{2 \times N}=\frac{\phi_{L}}{10}=18^{\circ} .
$$

Step 3: Use the $\mathrm{Q}$ and $\mathrm{N}$ values to determine the $L_{L}$ and $C_{L}$ product. Thus,

$$
Q=\frac{N}{2 \pi}\left(\frac{1}{\sqrt{L_{L} C_{L}}}\right)
$$


Table 1. Data for Linecalc for a Microstrip Transmission Line.

\begin{tabular}{|l|c|}
\hline Substrate Dielectric Constant $\left(\epsilon_{r}\right)$ & 3.38 \\
\hline Substrate Thickness $(\mathrm{H})$ & $0.813 \mathrm{~mm}$ \\
\hline Loss Tangent $(\operatorname{TanD})$ & 0.0021 \\
\hline Copper Thickness $(\mathrm{T})$ & $0.0356 \mathrm{~mm}$ \\
\hline Impedance & $50 \Omega$ \\
\hline Trace Length & Set at $24.5^{\circ}$ as a starting point \\
\hline
\end{tabular}

and

$$
L_{L} C_{L}=\left(\frac{N}{2 \pi Q}\right)^{2}
$$

The results are

$$
L_{L} C_{L}=9.2524 \times 10^{-20} \text { seconds }^{2}
$$

Step 4: Solve for $L_{L}$ and $C_{L}$ with the $L_{L} C_{L}$ product and the selected characteristic impedance $Z_{0 L}=50 \Omega$ yields:

$$
L_{L}=1.5209 \times 10^{-08}=15 \mathrm{nH}
$$

and

$$
C_{L}=6.0836 \times 10^{-12}=6 \mathrm{pF} .
$$

Step 5: Use $P f_{1}$ or $P f_{2}$ to obtain the electrical length of the right-handed transmission line (RHTL).

The electrical length was calculated to be $24.5^{\circ}$. With the electrical length determined, the length and width of the right-handed transmission line is calculated. Linecalc, from ADS, was used to determine these values. The material parameters were obtained from the data sheet of the printed circuit board (pcb) material that was selected for this project (Rogers RO4003C) as shown in Tables 1 and 2. Linecalc gave the results for the two transmission lines in Table 4.

The line impedance and capacitance values for the different phase shifts were 
Table 2. Data for Linecalc for a Coplanar Waveguide with Ground (CPWG).

\begin{tabular}{|l|c|}
\hline Substrate Dielectric Constant $\left(\epsilon_{r}\right.$ & 3.38 \\
\hline Substrate Thickness $(\mathrm{H})$ & $0.813 \mathrm{~mm}$ \\
\hline Gap Spacing $(\mathrm{G})$ & $1 \mathrm{~mm}$ \\
\hline Loss Tangent $($ TanD) & 0.0021 \\
\hline Copper Thickness $(\mathrm{T})$ & $0.0356 \mathrm{~mm}$ \\
\hline Impedance & $50 \Omega$ \\
\hline Trace Length & Set at $24.5^{\circ}$ as a starting point \\
\hline
\end{tabular}

Table 3. Physical Parameters for the Transmission Lines.

\begin{tabular}{|c|c|c|}
\hline & Microstrip & CPWG \\
\hline Line Width & $1.78 \mathrm{~mm}$ & $1.77 \mathrm{~mm}$ \\
\hline Line Length & $13.4 \mathrm{~mm}$ & $13 \mathrm{~mm}$ \\
\hline Gap & - & $1 \mathrm{~mm}$ \\
\hline
\end{tabular}

calculated at the two center frequencies $\left(f_{1}=915 \mathrm{MHz}\right.$ and $\left.f_{2}=2450 \mathrm{MHz}\right)$ using (3.20) through $(3.24)$. The phase shift values went from $10^{\circ}$ to $45^{\circ}$ in $5^{\circ}$ steps. The impedance and electrical length of the transmission line was set at $50 \Omega$ and $90^{\circ}$. The admittance of the transmission line with a phase shift is given by

$$
Y_{C}=Y_{0} \sec \frac{\text { phaseshift }}{2} \sin \beta l,
$$

and the shunt addmittance is

$$
B_{L 1}=Y_{0} \cos \beta l \sec \frac{\text { phaseshift }}{2}+\tan \frac{\text { phaseshift }}{2} .
$$

The reactance of the phase shift capacitance is

$$
X_{L 1}=\frac{1}{B_{L 1}} \text {. }
$$


Table 4. Capacitance Values for Different Phase Shifts at $915 \mathrm{MHz}$.

\begin{tabular}{|c|c|c|c|c|c|c|c|c|}
\hline Phase Shift & $10^{\circ}$ & $15^{\circ}$ & $20^{\circ}$ & $25^{\circ}$ & $30^{\circ}$ & $35^{\circ}$ & $40^{\circ}$ & $45^{\circ}$ \\
\hline Yc $(\mathrm{S})$ & 0.0201 & 0.0202 & 0.0203 & 0.0205 & 0.0207 & 0.0210 & 0.0213 & 0.0216 \\
\hline Zc $(\Omega)$ & 49.8 & 49.6 & 49.2 & 48.8 & 48.3 & 47.7 & 47.0 & 46.2 \\
\hline BL1 $(\mathrm{S})$ & 0.0017 & 0.0026 & 0.0035 & 0.0044 & 0.0054 & 0.0063 & 0.0073 & 0.0083 \\
\hline XL1 $(\Omega)$ & 571.5 & 379.8 & 283.6 & 225.5 & 186.6 & 158.6 & 137.4 & 120.7 \\
\hline C1 $(\mathrm{pF})$ & 0.304 & 0.458 & 0.613 & 0.771 & 0.932 & 1.097 & 1.266 & 1.441 \\
\hline
\end{tabular}

Table 5. Capacitance Values for Different Phase Shifts at $2450 \mathrm{MHz}$.

\begin{tabular}{|c|c|c|c|c|c|c|c|c|}
\hline Phase Shift & $10^{\circ}$ & $15^{\circ}$ & $20^{\circ}$ & $25^{\circ}$ & $30^{\circ}$ & $35^{\circ}$ & $40^{\circ}$ & $45^{\circ}$ \\
\hline $\mathrm{C} 2(\mathrm{pF})$ & 0.114 & 0.171 & 0.229 & 0.288 & 0.348 & 0.410 & 0.473 & 0.538 \\
\hline
\end{tabular}

The capacitance values at the two center frequencies are

$$
C_{1}=\frac{1}{2 \pi f_{1} X_{L 1}},
$$

and

$$
C_{2}=\frac{1}{2 \pi f_{2} X_{L 1}} .
$$

The results are listed in Tables 4 and 5 .

A single varactor might not be able to yield capacitance values this small. A fixed value capacitor in series with the varactor diode might be needed to obtain these capacitance values. This situation will be examined completely in the next chapter. 


\section{CHAPTER 4. SIMULATION}

The simulation software that was used is Agilent's Advanced Design System (ADS). Specifically, the linear component simulation engine and the 2.5-D electromagnetic simulation engine were used.

\subsection{Ideal Components}

The first step was to build the simulation schematic using ideal components (supplied with ADS). Note that on this schematic the reactive loads for the phase shift are disabled. This was done to ensure that the left-handed transmission line was operating correctly.

Since the two frequency bands needed different lengths of microstrip or coplanar transmission lines, seperate outputs were used. The lengths of the transmission lines (TL2 and TL3 in Figure 15) were optimized for a 10-degree phase shift at the desired frequencies of 920 and $2450 \mathrm{MHz}$. From Figure 16, Figure 17, and Table 6, the phase shift (phase of $S_{21}$ ), insertion loss (magnitude of $S_{21}$ ), and the return loss (magnitude of $\left.S_{11}\right)$ all meet expectations.

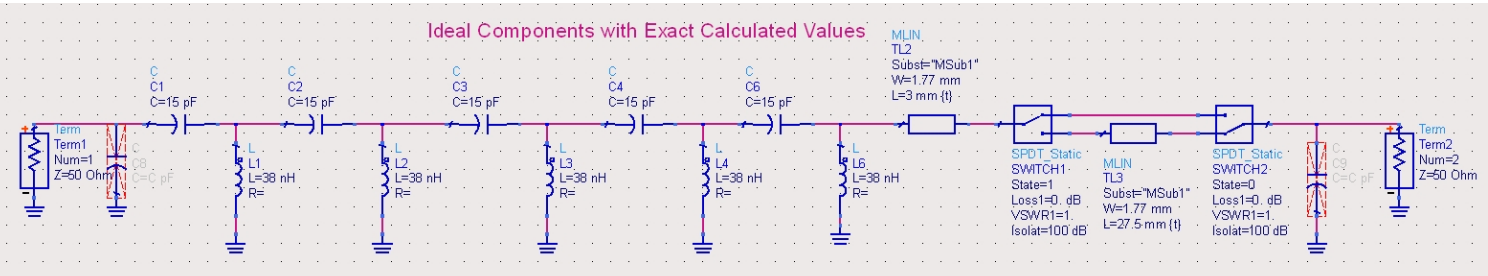

Figure 15. ADS Phase Shifter - Ideal Components with Exact Values. 

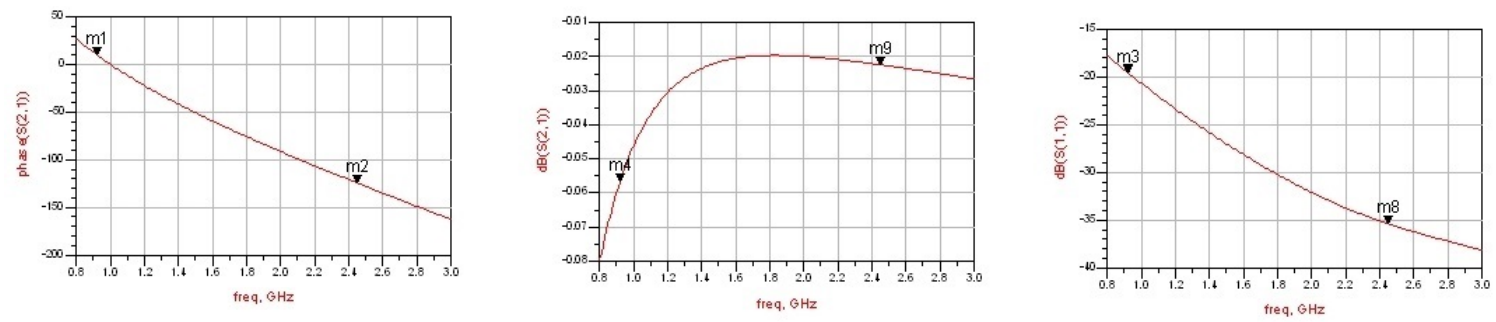

Figure 16. $920 \mathrm{MHz}$ Output: Phase of $S_{21}$, Magnitude of $S_{21}$, and Magnitude of $S_{11}$.
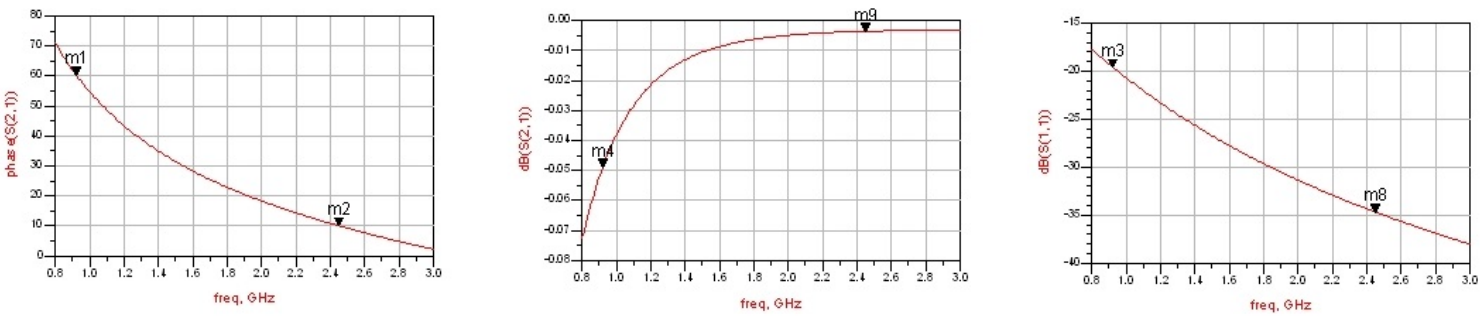

Figure 17. $2450 \mathrm{MHz}$ Output: Phase of $S_{21}$, Magnitude of $S_{21}$, and Magnitude of $S_{11}$.

Table 6. Phase and Magnitude Readings for the $920 \mathrm{MHz}$ and $2450 \mathrm{MHz}$ Outputs.

\begin{tabular}{|c|c|c|}
\hline \multicolumn{3}{|c|}{ 920-MHz Output Port } \\
\hline phase of $S_{21}$ & 9.999 degrees at $920 \mathrm{MHz}$ & -124.5 degrees at $2450 \mathrm{MHz}$ \\
\hline magnitude of $S_{21}$ & $-0.06 \mathrm{~dB}$ at $920 \mathrm{MHz}$ & $-0.022 \mathrm{~dB}$ at $2450 \mathrm{MHz}$ \\
\hline magnitude of $S_{11}$ & $-19.5 \mathrm{~dB}$ at $920 \mathrm{MHz}$ & $-35.4 \mathrm{~dB}$ at $2450 \mathrm{MHz}$ \\
\hline \hline \multicolumn{3}{|c|}{$2450-\mathrm{MHz}$ Output Port } \\
\hline phase of $S_{21}$ & 60.4 degrees at $920 \mathrm{MHz}$ & 10 degrees at $2450 \mathrm{MHz}$ \\
\hline magnitude of $S_{21}$ & $-0.05 \mathrm{~dB}$ at $920 \mathrm{MHz}$ & $-0.004 \mathrm{~dB}$ at $2450 \mathrm{MHz}$ \\
\hline magnitude of $S_{11}$ & $-19.6 \mathrm{~dB}$ at $920 \mathrm{MHz}$ & $-35 \mathrm{~dB}$ at $2450 \mathrm{MHz}$ \\
\hline
\end{tabular}

\subsection{Real Components}

All components have parasitic elements associated with them. Also, something that is not commonly recognized is that the printed circuit board will also add parasitic components to the circuit. The parasitic elements of the components are included by either measuring the individual components or using the manufacturer's measurements. Some of the problems with measuring the individual components are:

- Availablility of the appropriate test equipment. Ideally this would be an 
impedance analyzer.

- The impedance analyzer having the necessary frequency range and output power.

- Designing the appropriate test jigs.

- De-embedding the test jigs.

- Measuring each component.

- Applying the de-embedding correction to each measurement.

If many components are used to complete the design, this can be a very long, tedious, and error-prone procedure. Sometimes a network analyzer is used for parameter extraction. However, since the components used for this design are capacitors and inductors, the resulting plot on the network analyzer will be at the edge (or very near the edge) of the Smith Chart. The accuracy of the Smith Chart decreases significantly at the edge, making the accuracy of the measurements questionable. Component manufacturers have developed proprietary methods that allow them to use a network analyzer for accurate component characterization. Murata Electronics components were used for the design (see Figure 18). The accuracy of these models is examined in Appendix J.

The lengths of the transmission lines (TL4 and TL7 in Figure 18) were optimized for a 10 degree phase shift at the desired frequencies of 920 and $2450 \mathrm{MHz}$. From Figure 19, Figure 20, and Table 7, the phase shift (phase of $S_{21}$ ), insertion loss (magnitude of $S_{21}$ ), and the return loss (magnitude of $S_{11}$ in dB) all met expectations. Note that on this schematic the reactive loads for the phase shift are disabled. This was done to ensure that the left-handed transmission line was operating correctly. 


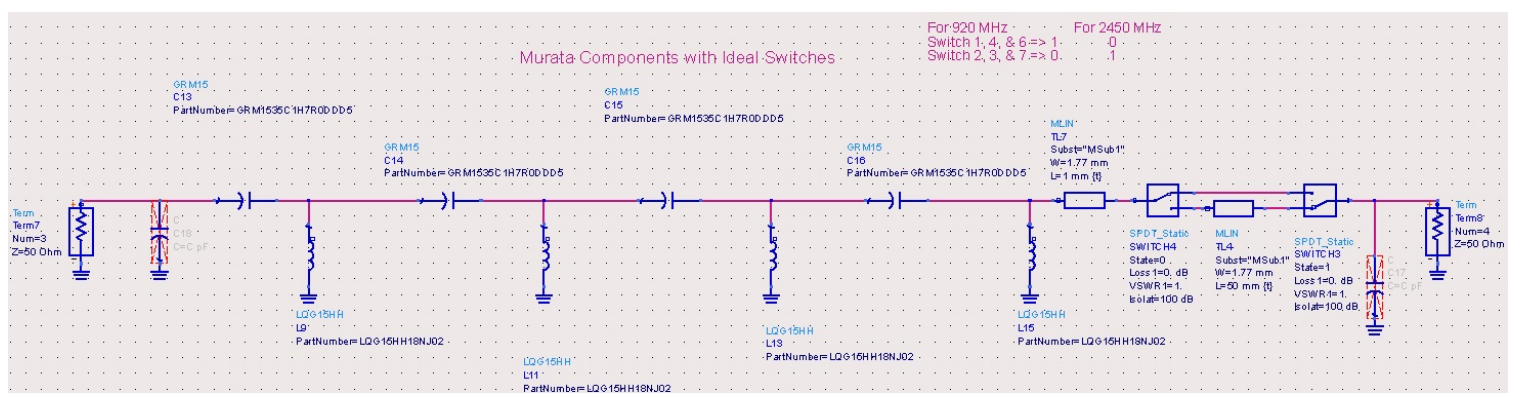

Figure 18. ADS Phase Shifter - Murata Components.
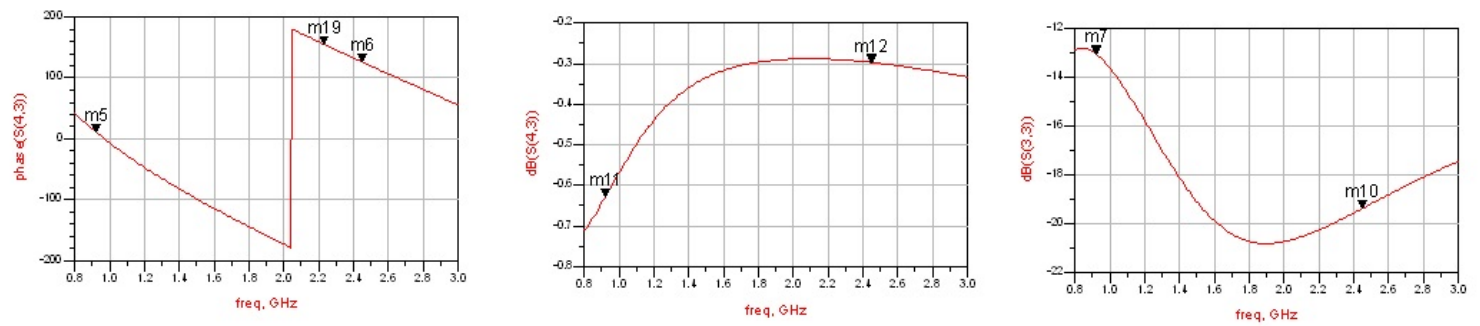

Figure 19. $920 \mathrm{MHz}$ Output: Phase of $S_{21}$, Magnitude of $S_{21}$, and Magnitude of $S_{11}$.
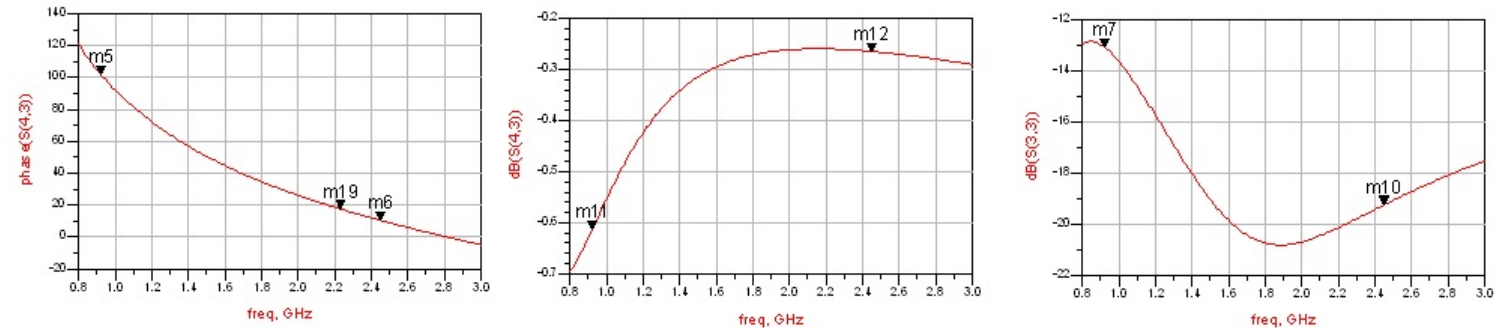

Figure 20. $2450 \mathrm{MHz}$ Output: Phase of $S_{21}$, Magnitude of $S_{21}$, and Magnitude of $S_{11}$.

Table 7. Phase and Magnitude Readings for the $920 \mathrm{MHz}$ and $2450 \mathrm{MHz}$ Outputs.

\begin{tabular}{|c|c|c|}
\hline \multicolumn{3}{|c|}{$920 \mathrm{MHz}$ Output Port } \\
\hline phase of $S_{21}$ & 10.5 degrees at $920 \mathrm{MHz}$ & 125.9 degrees at $2450 \mathrm{MHz}$ \\
\hline magnitude of $S_{21}$ & $-0.63 \mathrm{~dB}$ at $920 \mathrm{MHz}$ & $-0.26 \mathrm{~dB}$ at $2450 \mathrm{MHz}$ \\
\hline magnitude of $S_{11}$ & $-13 \mathrm{~dB}$ at $920 \mathrm{MHz}$ & $-19.4 \mathrm{~dB}$ at $2450 \mathrm{MHz}$ \\
\hline \hline \multicolumn{3}{|c|}{$2450 \mathrm{MHz}$ Output Port } \\
\hline phase of $S_{21}$ & 102.2 degrees at $920 \mathrm{MHz}$ & 10.4 degrees at $2450 \mathrm{MHz}$ \\
\hline magnitude of $S_{21}$ & $-0.61 \mathrm{~dB}$ at $920 \mathrm{MHz}$ & $-0.26 \mathrm{~dB}$ at $2450 \mathrm{MHz}$ \\
\hline magnitude of $S_{11}$ & $-13.1 \mathrm{~dB}$ at $920 \mathrm{MHz}$ & $-19.2 \mathrm{~dB}$ at $2450 \mathrm{MHz}$ \\
\hline
\end{tabular}




\subsection{Complete Phase Shifter}

The next step is to add the shunt varactor diodes and determine the phase shift, insertion loss, and return loss for different bias votages (see Figure 21). The manufacturer's (Infineon) data was used to simulate the varactor diodes. At selected reverse bias voltages, $S_{11}$ was measured. The frequency range of the measurements was from $50 \mathrm{MHz}$ to $6 \mathrm{GHz}$. As seen from Figure 22, this varactor diode has a capacitance range of approximately 1 to $2.5 \mathrm{pF}$. Based on the initial design parameters, this capacitance range is too large. To reduce the capacitance range a, $1 \mathrm{pF}$ capacitor was placed in series with the varactor diode. The simulation results are shown in Tables 8 and 9

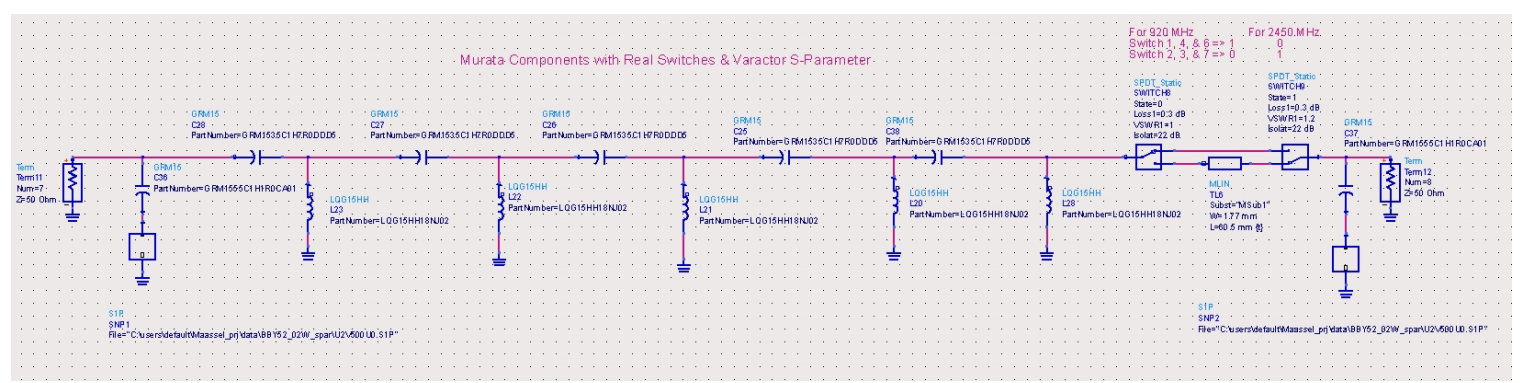

Figure 21. Simulation Schematic with Murata Components and S-Parameter Files for the Varactor Diodes. 


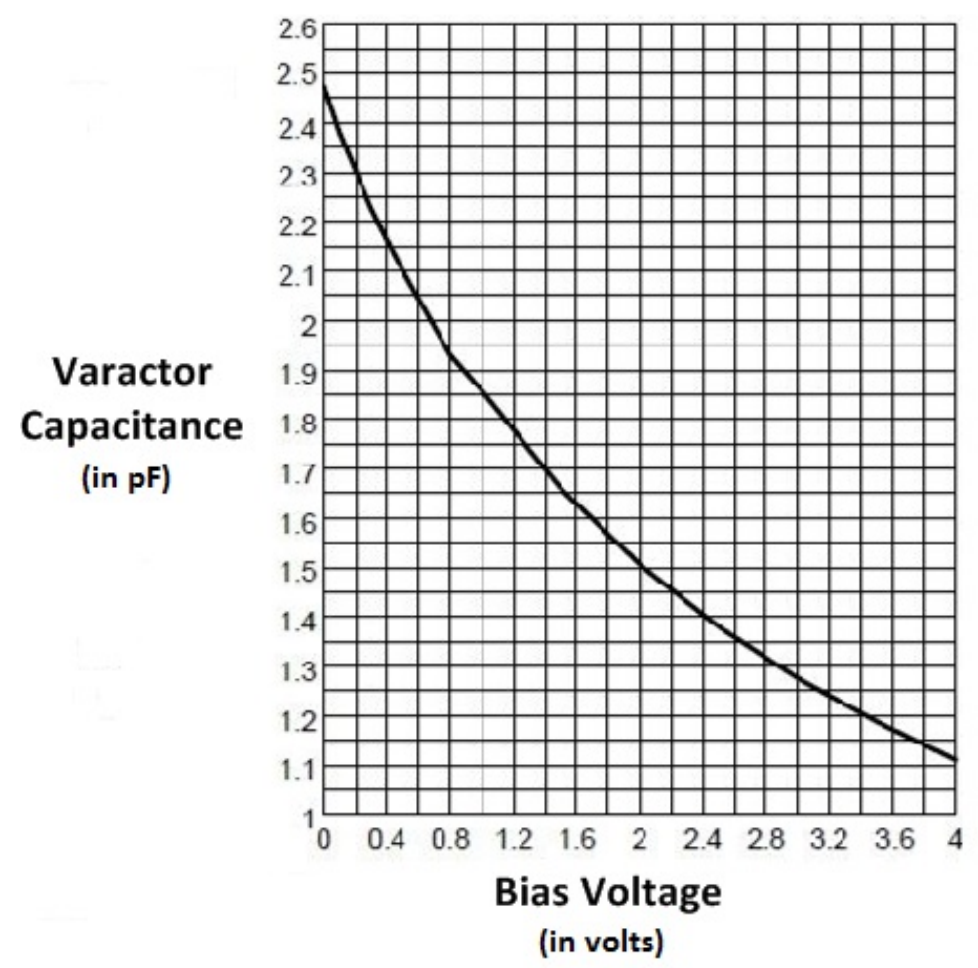

Figure 22. The Capacitance versus Reverse Bias Voltage for the Varactor Diode.

Table 8. Phase Shift, Insertion, and Return Loss for values of Reverse Bias Voltages.

\begin{tabular}{|c|c|c|c|}
\hline \multicolumn{5}{|c|}{$920-\mathrm{MHz}$ Output } \\
\hline Bias Voltage & Phase Shift & Insertion Loss & Return Loss \\
\hline $0.5 \mathrm{~V}$ & $5.4^{\circ}$ & $1.67 \mathrm{~dB}$ & $9.97 \mathrm{~dB}$ \\
\hline $0.6 \mathrm{~V}$ & $5.6^{\circ}$ & $1.66 \mathrm{~dB}$ & $10 \mathrm{~dB}$ \\
\hline $0.7 \mathrm{~V}$ & $5.7^{\circ}$ & $1.65 \mathrm{~dB}$ & $10.1 \mathrm{~dB}$ \\
\hline $0.8 \mathrm{~V}$ & $5.8^{\circ}$ & $1.65 \mathrm{~dB}$ & $10.1 \mathrm{~dB}$ \\
\hline $0.9 \mathrm{~V}$ & $5.9^{\circ}$ & $1.65 \mathrm{~dB}$ & $10.1 \mathrm{~dB}$ \\
\hline $1.0 \mathrm{~V}$ & $6^{\circ}$ & $1.64 \mathrm{~dB}$ & $10.2 \mathrm{~dB}$ \\
\hline $1.5 \mathrm{~V}$ & $6.5^{\circ}$ & $1.62 \mathrm{~dB}$ & $10.3 \mathrm{~dB}$ \\
\hline $2.5 \mathrm{~V}$ & $7.3^{\circ}$ & $1.6 \mathrm{~dB}$ & $10.6 \mathrm{~dB}$ \\
\hline $3 \mathrm{~V}$ & $7.6^{\circ}$ & $1.6 \mathrm{~dB}$ & $10.7 \mathrm{~dB}$ \\
\hline $3.5 \mathrm{~V}$ & $7.96^{\circ}$ & $1.56 \mathrm{~dB}$ & $10.8 \mathrm{~dB}$ \\
\hline $4 \mathrm{~V}$ & $8.29^{\circ}$ & $1.6 \mathrm{~dB}$ & $10.9 \mathrm{~dB}$ \\
\hline $4.5 \mathrm{~V}$ & $8.6^{\circ}$ & $1.54 \mathrm{~dB}$ & $11 \mathrm{~dB}$ \\
\hline $5 \mathrm{~V}$ & $8.96^{\circ}$ & $1.53 \mathrm{~dB}$ & $11.1 \mathrm{~dB}$ \\
\hline $6 \mathrm{~V}$ & $9.6^{\circ}$ & $1.5 \mathrm{~dB}$ & $11.4 \mathrm{~dB}$ \\
\hline $7 \mathrm{~V}$ & $10.1^{\circ}$ & $1.49 \mathrm{~dB}$ & $11.5 \mathrm{~dB}$ \\
\hline
\end{tabular}


Table 9. Phase Shift, Insertion, and Return Loss for values of Reverse Bias Voltages.

\begin{tabular}{|c|c|c|c|}
\hline \multicolumn{4}{|c|}{$2450-\mathrm{MHz}$ Output } \\
\hline Bias Voltage & Phase Shift & Insertion Loss & Return Loss \\
\hline $0.5 \mathrm{~V}$ & $-15.6^{\circ}$ & $2.5 \mathrm{~dB}$ & $4.2 \mathrm{~dB}$ \\
\hline $0.6 \mathrm{~V}$ & $-15.3^{\circ}$ & $2.5 \mathrm{~dB}$ & $4.3 \mathrm{~dB}$ \\
\hline $0.7 \mathrm{~V}$ & $-14.9^{\circ}$ & $2.4 \mathrm{~dB}$ & $4.3 \mathrm{~dB}$ \\
\hline $0.8 \mathrm{~V}$ & $-14.6^{\circ}$ & $2.4 \mathrm{~dB}$ & $4.4 \mathrm{~dB}$ \\
\hline $0.9 \mathrm{~V}$ & $-14.3^{\circ}$ & $2.37 \mathrm{~dB}$ & $4.4 \mathrm{~dB}$ \\
\hline $1.0 \mathrm{~V}$ & $-14^{\circ}$ & $2.34 \mathrm{~dB}$ & $4.5 \mathrm{~dB}$ \\
\hline $1.5 \mathrm{~V}$ & $-12.7^{\circ}$ & $2.19 \mathrm{~dB}$ & $4.7 \mathrm{~dB}$ \\
\hline $2.5 \mathrm{~V}$ & $-10.4^{\circ}$ & $1.95 \mathrm{~dB}$ & $5.2 \mathrm{~dB}$ \\
\hline $3 \mathrm{~V}$ & $-9.4^{\circ}$ & $1.85 \mathrm{~dB}$ & $5.4 \mathrm{~dB}$ \\
\hline $3.5 \mathrm{~V}$ & $-8.5^{\circ}$ & $1.75 \mathrm{~dB}$ & $5.6 \mathrm{~dB}$ \\
\hline $4 \mathrm{~V}$ & $-7.5^{\circ}$ & $1.67 \mathrm{~dB}$ & $5.8 \mathrm{~dB}$ \\
\hline $4.5 \mathrm{~V}$ & $-6.6^{\circ}$ & $1.58 \mathrm{~dB}$ & $6 \mathrm{~dB}$ \\
\hline $5 \mathrm{~V}$ & $-5.6^{\circ}$ & $1.5 \mathrm{~dB}$ & $6.2 \mathrm{~dB}$ \\
\hline $6 \mathrm{~V}$ & $-3.6^{\circ}$ & $1.34 \mathrm{~dB}$ & $6.7 \mathrm{~dB}$ \\
\hline $7 \mathrm{~V}$ & $-2.4^{\circ}$ & $1.24 \mathrm{~dB}$ & $7 \mathrm{~dB}$ \\
\hline
\end{tabular}

The results for phase shift, insertion loss, and return loss are reasonable enough to proceed to the fabrication and testing stage.

\subsubsection{Infinitesimal Conditions}

Before the printed circuit board can be fabricated, the first infinitesimal condition (introduced in chapter 3 - the phase shift of each unit cell must be less the $90^{\circ}$ ) must be verified using (4.1) through (4.9)

$$
\begin{gathered}
f_{1}=920 \times 10^{6} \mathrm{~Hz}, \\
L=18 \times 10^{-9} \mathrm{H}, \\
C=7 \times 10^{-12} \mathrm{~F},
\end{gathered}
$$




$$
\begin{gathered}
Z_{0}=\sqrt{\frac{L}{C}} \\
w_{1}=2 \pi f_{1} \\
\text { Phase Shift at } 915 \mathrm{MHz}=-\tan ^{-1} \frac{w_{1}\left(\frac{L}{Z_{0}}+\left(C \times Z_{0}\right)\right)}{1-2 w_{1}^{2} L C}, \\
f_{2}=2450 \times 10^{6} \mathrm{~Hz}, \\
w_{2}=2 \pi f_{2},
\end{gathered}
$$

and

$$
\text { Phase Shift at } 2450 \mathrm{MHz}=-\tan ^{-1} \frac{w_{2}\left(\frac{L}{Z_{0}}+\left(C \times Z_{0}\right)\right)}{1-2 w_{2}^{2} L C} \text {. }
$$

The results were: phase shift at $915 \mathrm{MHz}=28.9^{\circ}$ and phase shift at 2450 $\mathrm{MHz}=10.5^{\circ}$. These phase shifts satisfy the requirement for the first infinitesimal condition. The second condition that needs to be met for the infinitesimal condition to be satisfied is that the physical size of the left-handed unit cell be less then $\frac{\lambda}{10}$. This condition will be examined when the fabrication of the printed circuit board is discussed in the next chapter. 


\section{CHAPTER 5. FABRICATION}

\subsection{Component Size Selection}

There are many different physical sizes of surface mount components available. These sizes can range from 0201 (length $=0.6 \mathrm{~mm}$ and width $=0.3 \mathrm{~mm}$ ) to 2512 (length $=6.3 \mathrm{~mm}$ and width $=3.2 \mathrm{~mm}$ ). For this circuit, a compromise was needed between being able to hand assemble the phase shifter and keeping the physical size less then one-tenth of a wavelength. The 0402 components (length $=1 \mathrm{~mm}$ and width $=0.5 \mathrm{~mm}$ ) were the size that was able to satisfy both of these requirements.

Now that the component size has been determined, the layout of the printed circuit board must be considered. Each component has what is called a footprint. This is used to lay out the printed circuit board to insure that the component is properly soldered to the board. From the Murata Electronics datasheet, the dimensions for the capacitor footprint and inductor footprint are given in Figures 23 and 24 and Tables 10 and 11.

\subsubsection{Capacitor and Inductor Footprints}

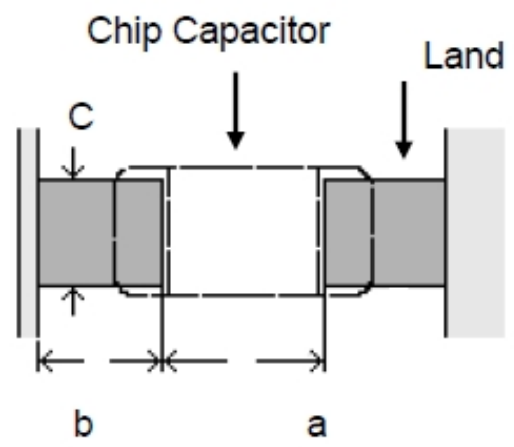

Figure 23. Footprint for the Capacitors

Table 10. Surface Mount Capacitor Footprint Dimensions.

\begin{tabular}{|c|c|c|}
\hline $\mathrm{a}$ & $\mathrm{b}$ & $\mathrm{c}$ \\
\hline $0.6 \sim 0.8 \mathrm{~mm}$ & $0.6 \sim 0.7 \mathrm{~mm}$ & $0.6 \sim 0.8 \mathrm{~mm}$ \\
\hline
\end{tabular}




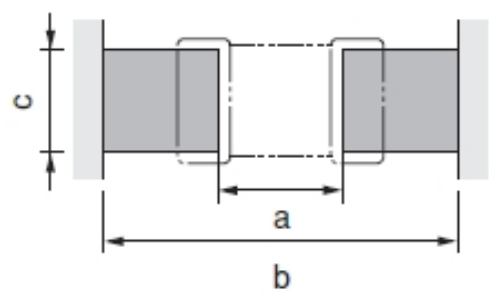

Figure 24. Footprint for the Inductors.

Table 11. Surface Mount Inductor Footprint Dimensions.

\begin{tabular}{|c|c|c|}
\hline $\mathrm{a}$ & $\mathrm{b}$ & $\mathrm{c}$ \\
\hline $0.6 \sim 0.8 \mathrm{~mm}$ & $1.8 \sim 2.2 \mathrm{~mm}$ & $0.6 \sim 0.8 \mathrm{~mm}$ \\
\hline
\end{tabular}

Now the actual layout of the printed circuit board can be examined. From an assembly prospective, the capacitors and inductors cannot be placed directly next to each other. There must be some space for the soldering pencil to touch both the component and the component pad located on the printed circuit board.

To determine the limit of the size of the unit cell, the wavelength of the highest operating frequency needs to be calculated. Since it is the wavelength of a transmission line, the parameters of the printed circuit board are required. The board material that was selected was Rogers 4003C. The neccessary parameters are given in Table 12.

Table 12. Printed Circuit Board Material Specifications.

\begin{tabular}{|c|c|}
\hline Dielectric Constant & 3.55 \\
\hline Loss Tangent & 0.0021 \\
\hline Substrate Thickness & $0.813 \mathrm{~mm}$ \\
\hline Copper Thickness & $0.035 \mathrm{~mm}$ \\
\hline
\end{tabular}

For this board material, a trace width of $1.77 \mathrm{~mm}$ will yield a $50-\Omega$ transmission line (for both microstrip and CPWG transmission lines). LineCalc was used to determine the wavelength of a $2500 \mathrm{MHz}$ signal (either for microstrip or coplanar waveguide with ground). For both types of transmission lines the wavelength at 2500 
MHs is approximately $72 \mathrm{~mm}$. Therefore one-tenth of a wavelength is $7.2 \mathrm{~mm}$. From the dimensions of the capacitors and inductors, it will be very easy to maintain the physical size of each unit cell to less then $7.2 \mathrm{~mm}$. This then satisfies the second and final requirement for the infinitesimal condition.

\subsection{Layout of the Printed Circuit Board}

The layout of the circuit board was done using the Layout Editor of ADS.

\subsubsection{Transmission Line Steps and Tapers}

The first issue that needed to be addressed is the difference in the component size and the width of the transmission lines. This was very significant for the SMA connector. The footprint for the SMA connector specified a line width of $1.3 \mathrm{~mm}$ while the transmission lines had a width of $1.77 \mathrm{~mm}$.

\subsubsection{Tapers}

For the printed circuit board material that was used, the impedance of both the microstrip transmission line and the coplanar waveguide (with ground) vary by the same amount when the width is changed. This information was verified by using several different line widths in LineCalc and determining the impedance. A simple simulation circuit (using coplanar waveguide with ground) was set up to determine the effect of the taper on the impedance of the transmission lines.

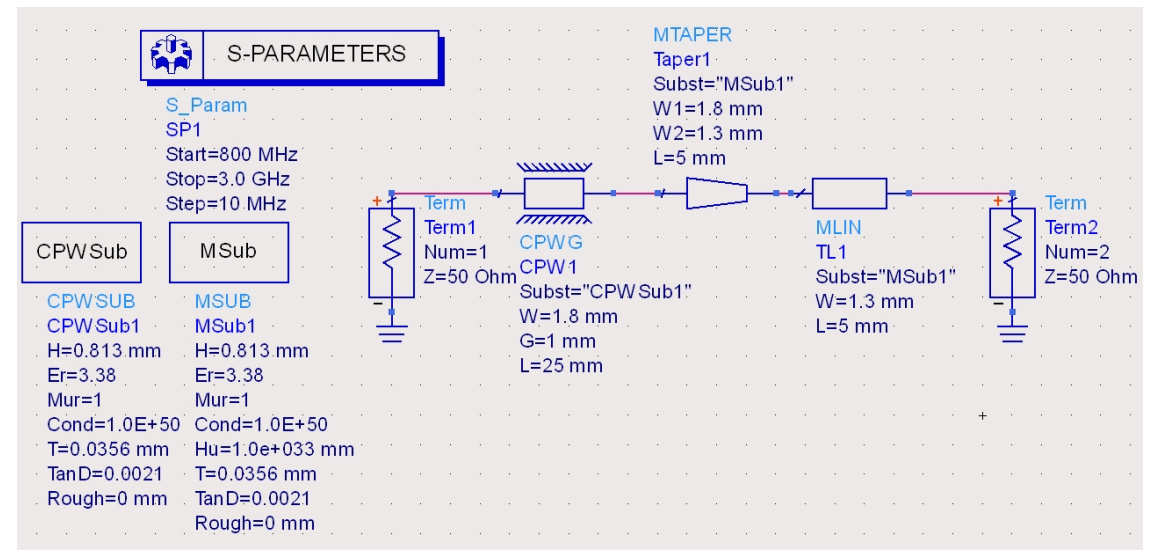

Figure 25. Simulation Schematic for the Taper from the CPWG to an SMA Connector. 

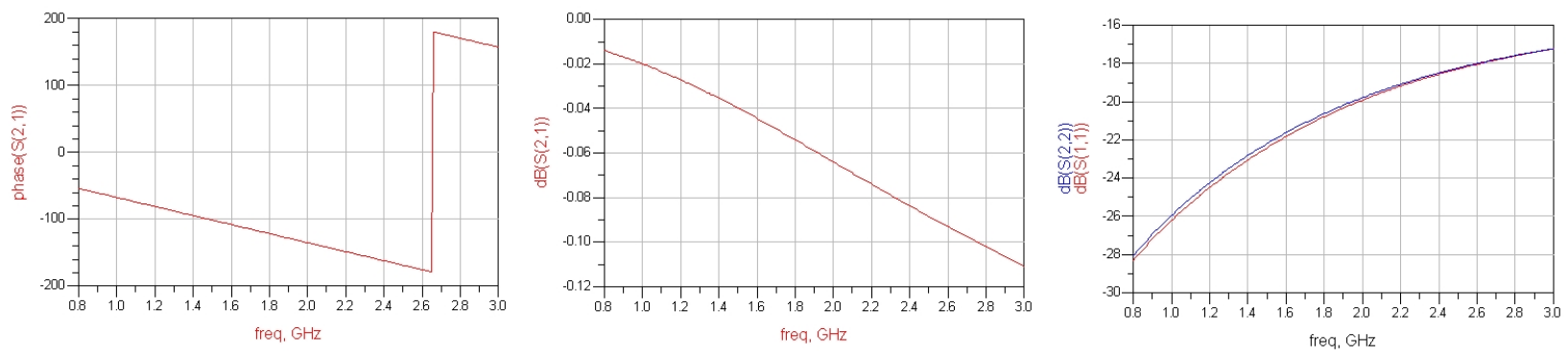

Figure 26. Simulation Output for the Taper from the CPWG to an SMA Connector.

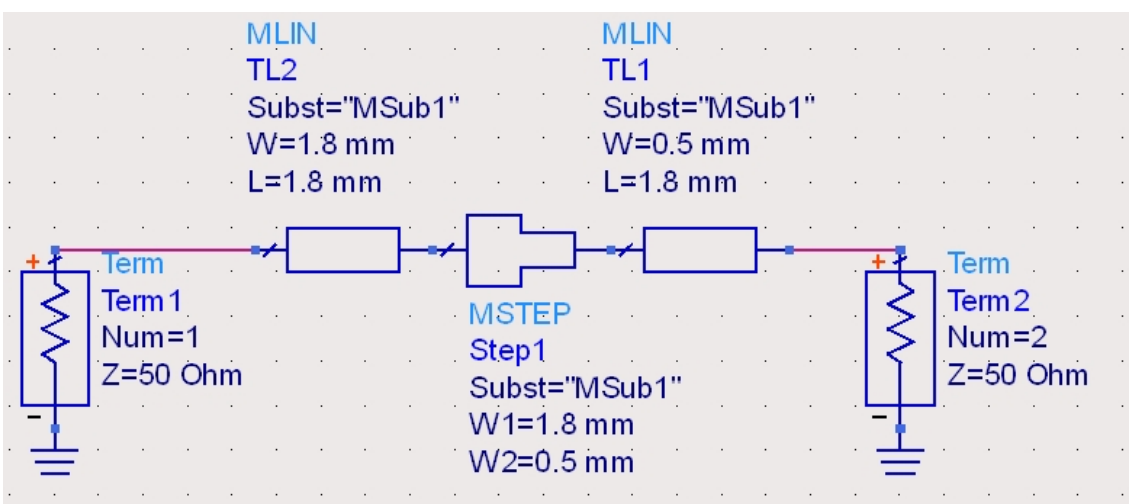

Figure 27. Simulation Schematic of a Microstrip Step to an 0402 Component.

As shown in Figure 26, the taper does not cause any serious discontinuites. The majority of the phase shift is caused by the $25 \mathrm{~mm}$ long CPWG. The insertion loss is negligible, and the graph of $S_{11}$ and $S_{22}$ indicates that the input and output (ports 1 and 2 respectively) impedance does not degrade significantly.

\subsubsection{Steps}

Since the goal is to keep the size of each Left-Handed Unit Cell small, tapers are not the best option for matching the width of the transmission lines to the capacitors or inductors. A simulation was performed using a microstrip step to determine if the abrupt change in line width would cause serious problems.

While the simulation did show that the phase of the step was larger then was desired, it was not a significant problem. The insertion loss is negligible and the graph of $S_{11}$ and $S_{22}$ indicates that the input and output (ports 1 and 2 respectively) 

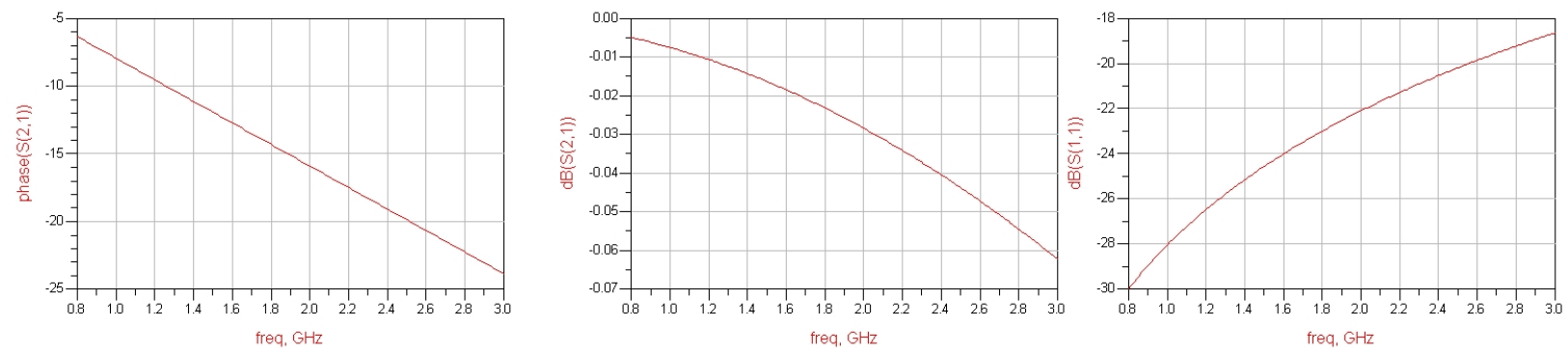

Figure 28. Simulation Output of a Microstrip Step to an 0402 Component.

impedance does not degrade significantly.

\subsection{Vias}

When dealing with CPWG, vias need to be used to connect the grounds on the top and bottom sides together. While there are nine different models for vias available in ADS, there are only two that will be considered. With the exception of the tapered via, these two models are representative of the different types of vias. The difference between these two models and the others has more to do with the pcb footprint than the actual modeling of the via. The models are:

- Libra Cylindrical Via Hole to Ground in Microstrip.

- Libra Cylindrical Via Hole in Microstrip.

Three other circuits were simulated to provide a reference point for the via models:

- Libra Microstrip Line Open-End Effect.

- Libra Microstrip Line Open-Circuited Stub.

- Libra Microstrip Line Short-Circuited Stub.

In all of these simulations, the total length of the transmission line or stub was set at $90^{\circ}$. A $90^{\circ}$-transmission line will rotate an impedance half-way around the 


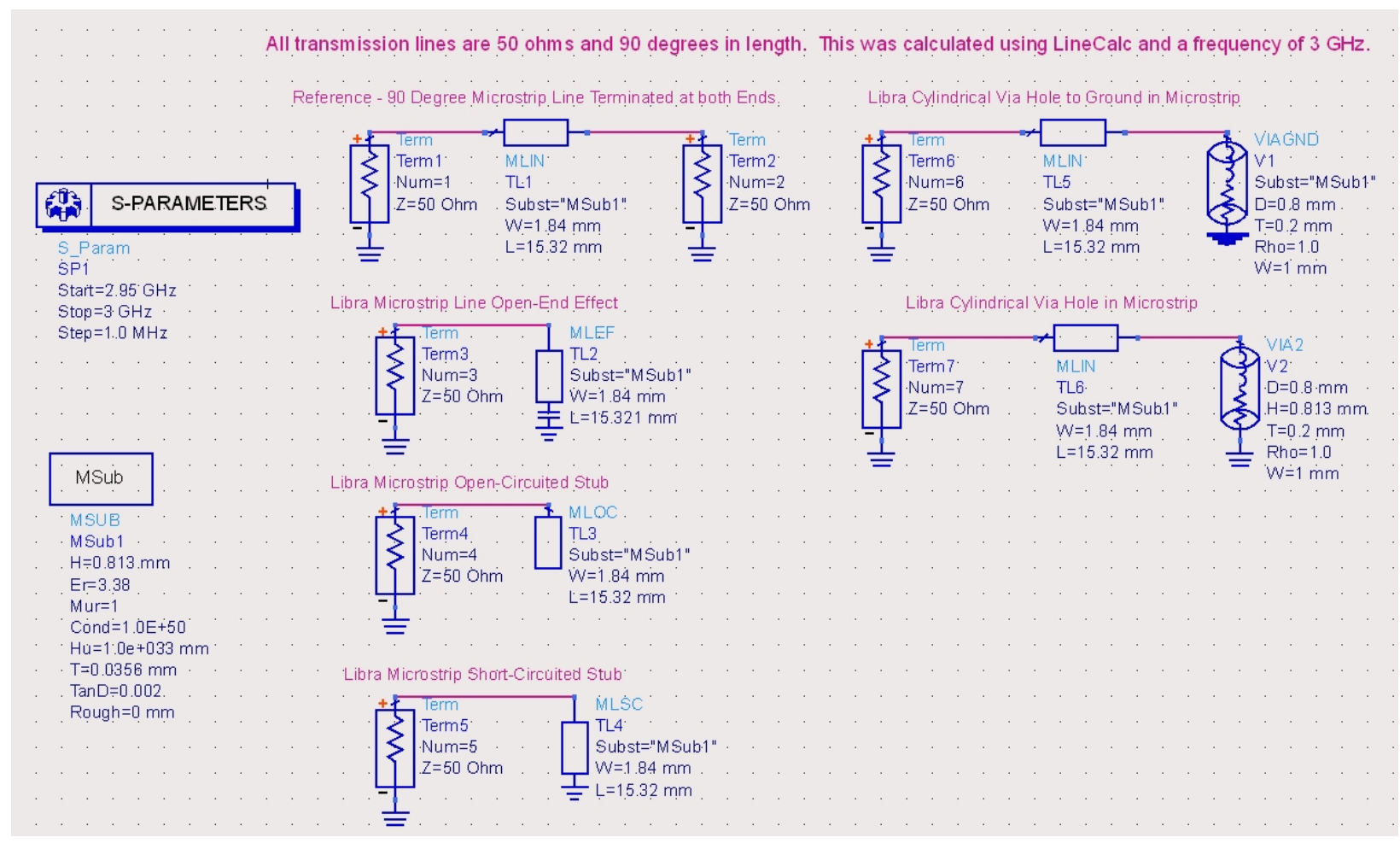

Figure 29. Simulation Schematic of the Different Via Models and the Reference Circuits.

Smith chart. Therefore, if a perfect short (or nearly perfect short) was placed at the end of this $90^{\circ}$ line, the result at the input would be an open circuit. This is the critera that was used to determine which via model yields the best results. The schematics and simulation results are shown in Figures 29 and 30. Based on the results of this simulation, either model provides an accurate grounded via.

\subsection{Thermal Reliefs}

Thermal reliefs are more of a manufacturing issue then a true design issue. Some of the capacitors and inductors will be soldered to the ground plane. The ground plane also acts as a thermal heatsink. When attempting to solder a component onto a ground plane, it will absorb so much heat that a good solder connection is not possible. Also in automated assembly lines, trying to solder components to ground planes could cause the component to tombstone (see Figure 31). To eliminate this 

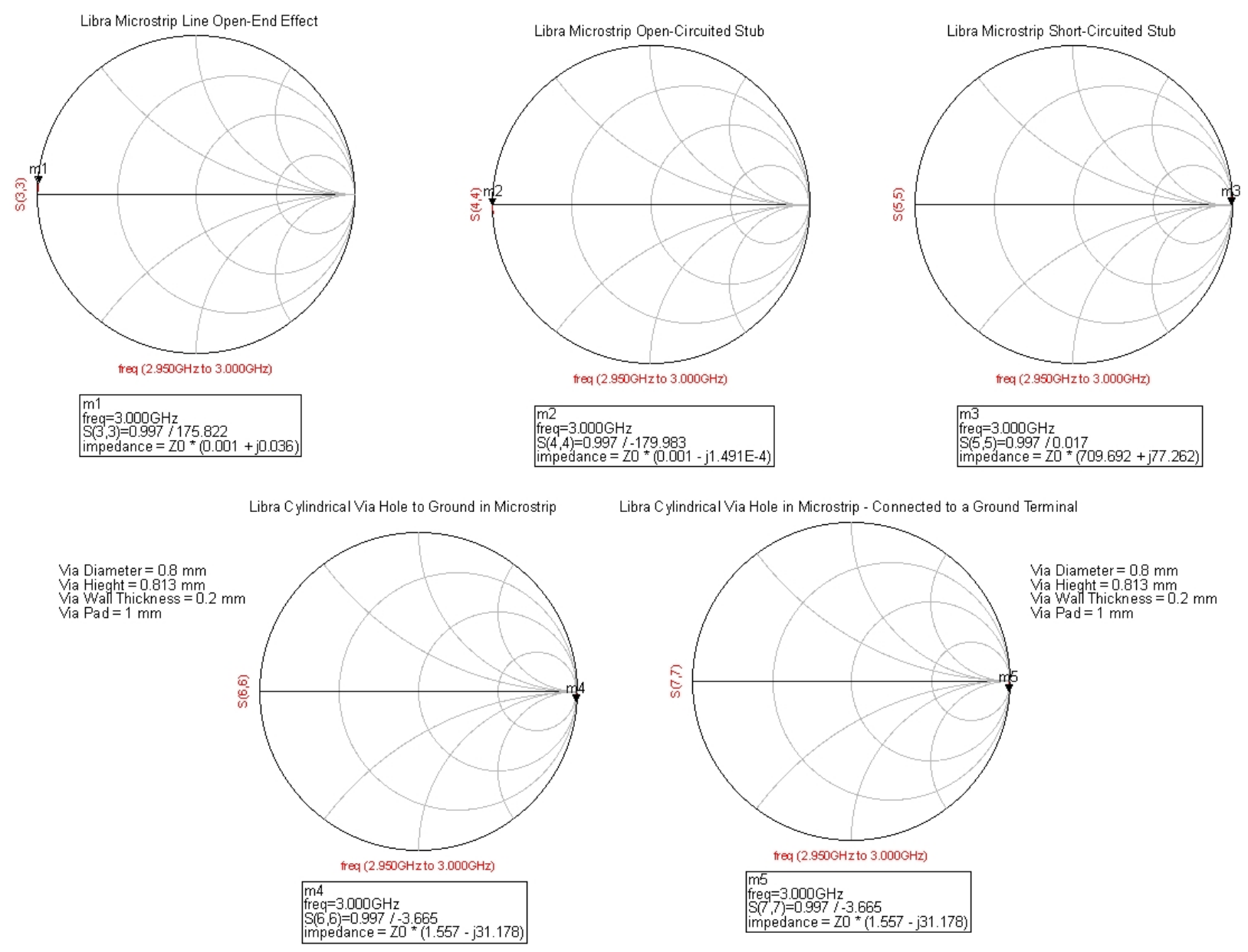

Figure 30. Simulation Output of the Different Via Models and the Reference Circuits.

problem, thermal reliefs need to be used (see Figure 32).

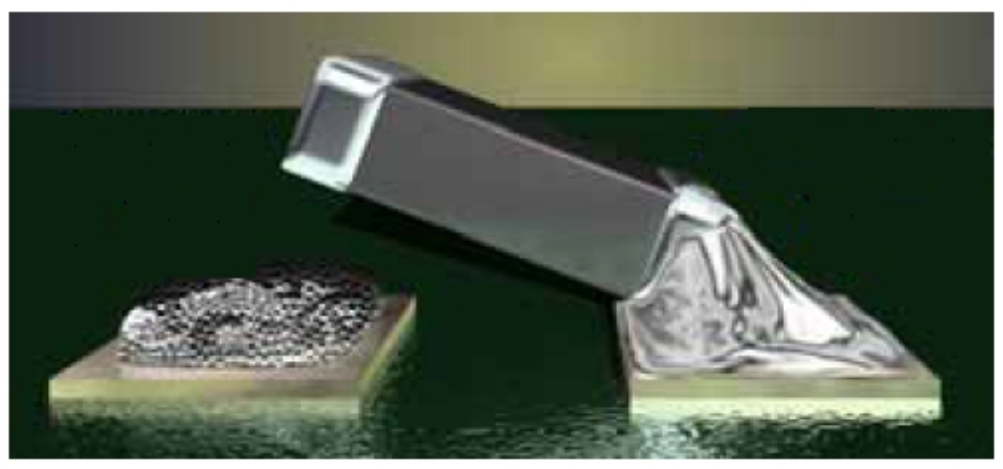

Figure 31. Tombstoned Passive Component. 


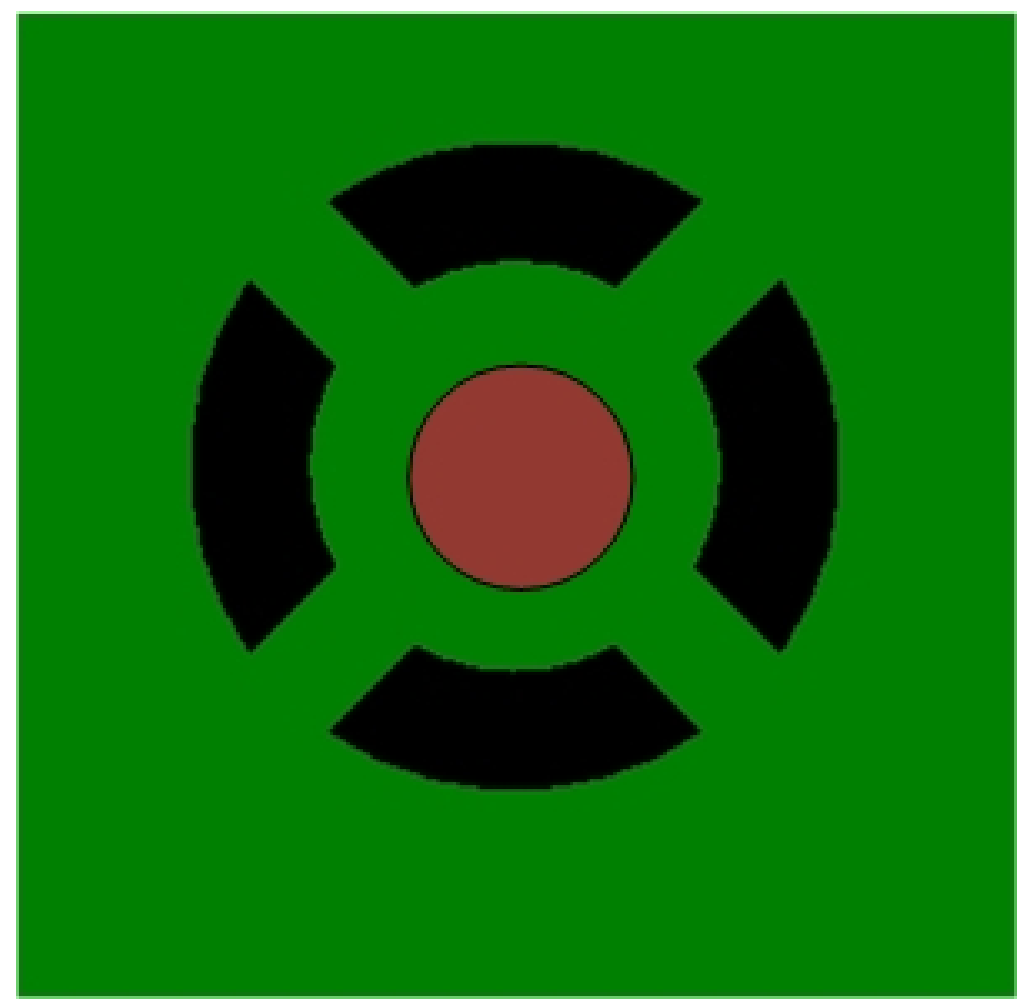

Figure 32. Thermal Relief.

In Figure 32, the orange is the via, the green is the ground plane and the black is where the ground plane has been removed. The trouble with thermal reliefs is the possiblity of increased inductance or, even worse, the possiblity of degradation due to small grounded transmission line stubs.

A literature search did not show any information about the electrical design or the electrical performance of thermal reliefs. Therefore, the next step is to determine how much effect the thermal reliefs will have on the circuit performance. One way to analyze these thermal reliefs is by using an electromagnetic simulator. ADS Momentum was used for this simulation. Momentum is a 2.5-D simulation engine.

\subsubsection{Momentum Evaluation of a Thermal Relief}

The starting point was a simulation with no thermal relieves. The Momentum layout is shown in Figure 33. Momentum alone cannot simulate lumped elements (a $50-\Omega$ resistor is used in the simulation); therefore, both Momentum and the Circuit 
Simulator were used. This is referred to as Cosimulation. The complete cosimulation schematic is shown in Figure 34. The simulation output is shown in Figure 35.

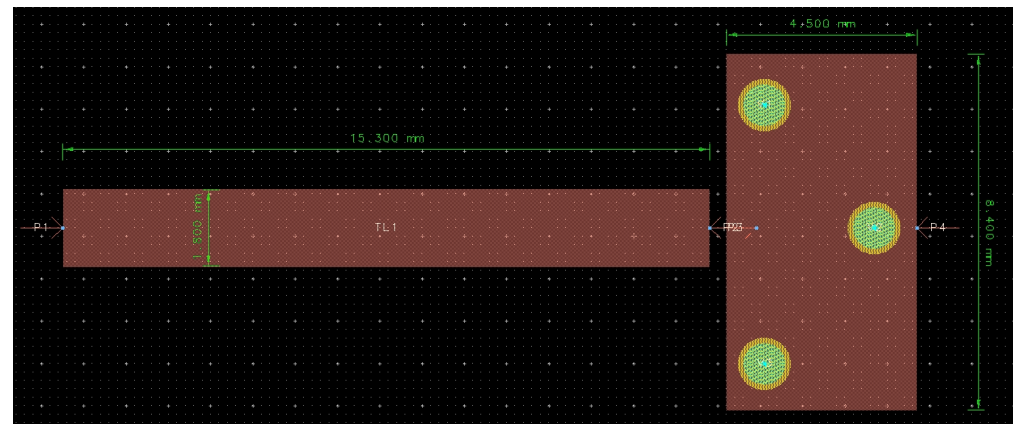

Figure 33. Momentum Layout without Thermal Reliefs.

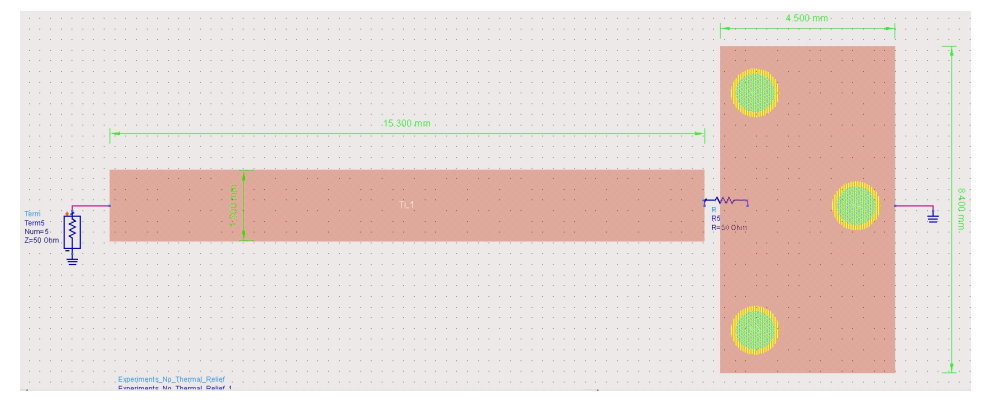

Figure 34. Cosimulation Schematic without Thermal Reliefs.

Since the highest operating frequency is the one which the thermal reliefs may start to cause problems, this will be the frequency that will be analyzed. Note: It is always very important to choose a frequency higher (and/or lower) then the highest (lowest) operating frequency. This will insure that there are no problems at or slightly beyond the band edges. This led to choosing $3 \mathrm{GHz}$ as the analysis frequency. From Figure $35 S_{55}($ at $3 \mathrm{GHz})$ is $0.038 \angle-40.46^{\circ}$, and the impedance is $52.9-\mathrm{j} 2.6 \Omega$. This is very close to having an excellent match to a $50-\Omega$ circuit.

Note: To speed up the simulation process, several different circuits were simulated at the same time which required using different port numbers for each circuit. 


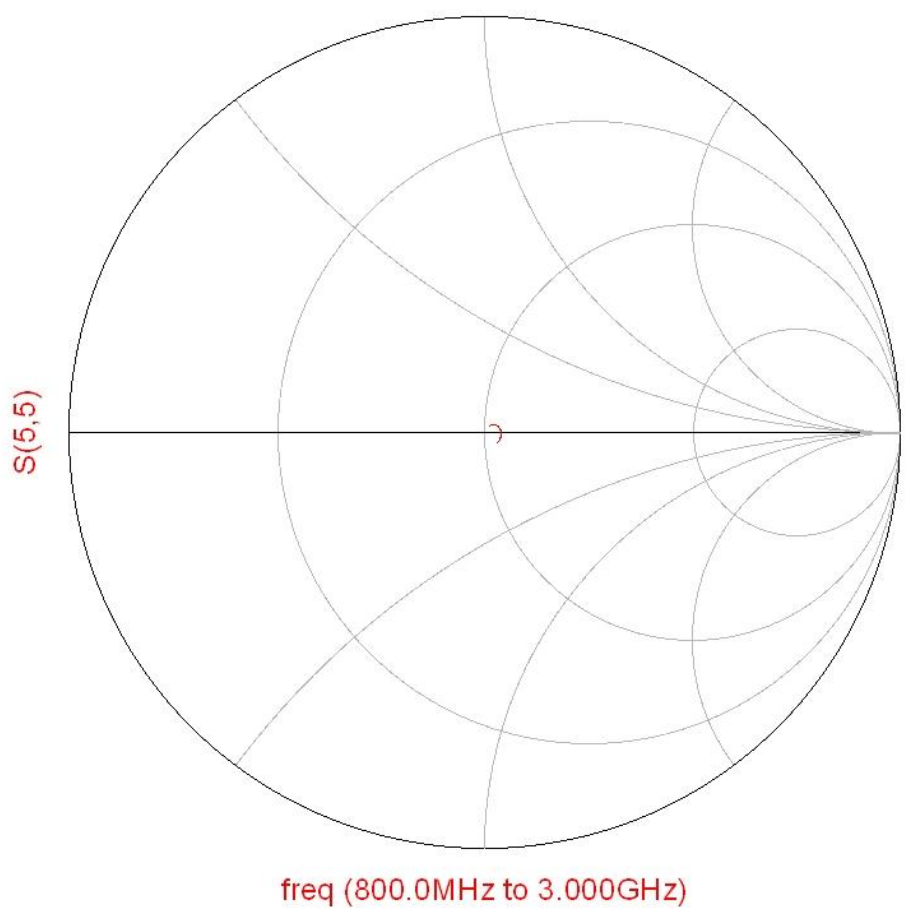

Figure 35. Cosimulation Output without Thermal Reliefs.

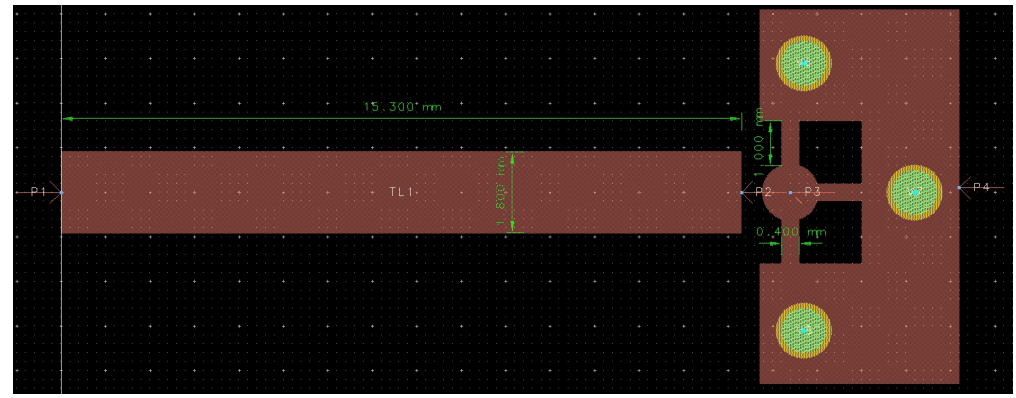

Figure 36. Momentum Layout with Thermal Reliefs.

The next step is to add the thermal reliefs. Figures 36, 37, and 38 show the Momentum layout, the Cosimulation schematic, and the simulation output respectively.

From Figure 38 (at $3 \mathrm{GHz}$ ) $S_{44}$ is 0.049 at $\angle-58.45^{\circ}$ and the impedance is $52.45-\mathrm{j} 4.4 \Omega$. While this is slightly worse then the no thermal relief simulation, it is still an excellent match to a $50-\Omega$ circuit. 


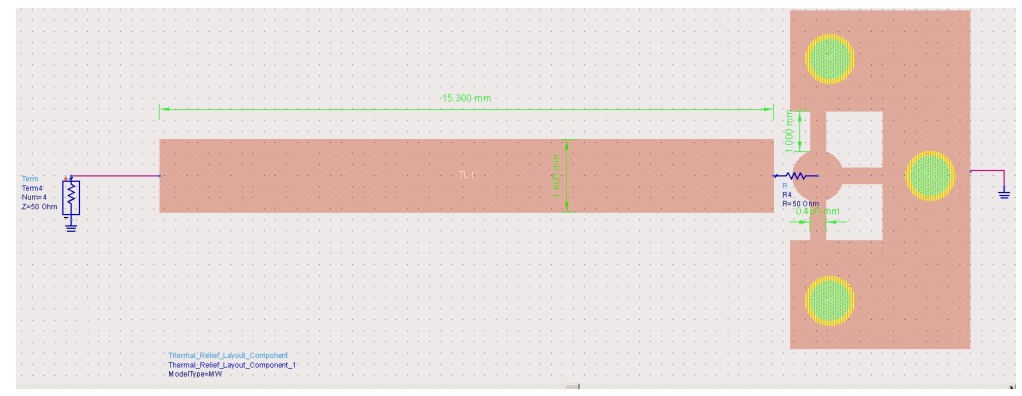

Figure 37. Cosimualation Schematic with Thermal Reliefs.

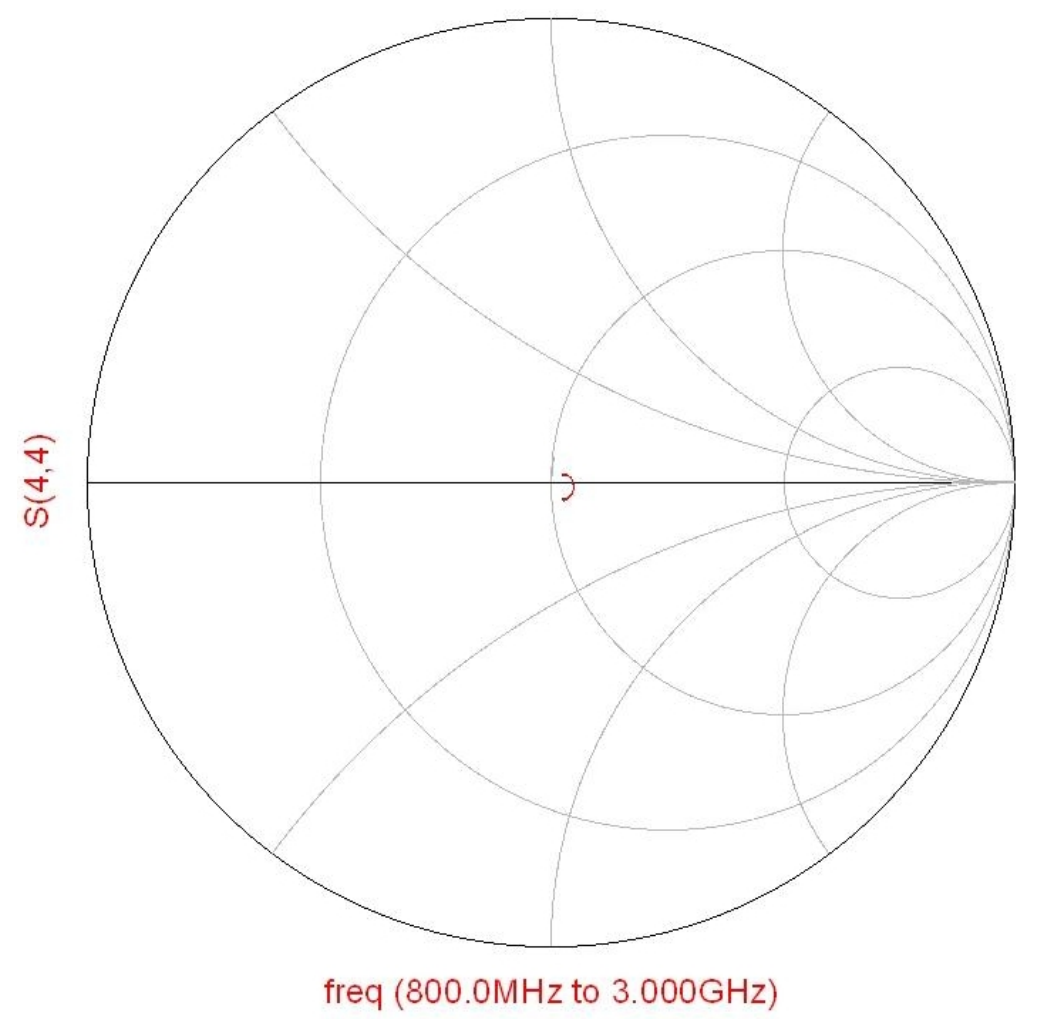

Figure 38. Cosimualation Output with Thermal Reliefs.

Stubs with lengths less that one-tenth of a wavelength have a minimal effect on a circuit. At $3 \mathrm{GHz}$, the wavelength (in the Rogers $4003 \mathrm{C}$ material) is $67 \mathrm{~mm}$. One-tenth of a wavelength would then be $6.7 \mathrm{~mm}$. It is difficult to vary the length of a stub when the circuit is modeled in Momentum. Therefore the circuit simulator was used to determine if there could be a problem with the thermal relief stub length. The length of the stub was varied from 1 to $10 \mathrm{~mm}$ in $1 \mathrm{~mm}$ steps. 


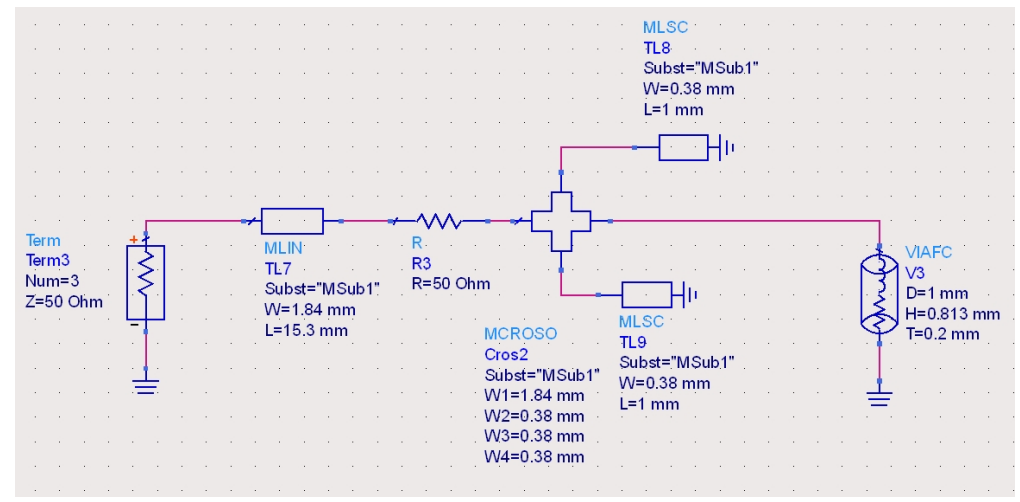

Figure 39. Simulation Schematic for Adjusting the Lengths of the Thermal Reliefs.

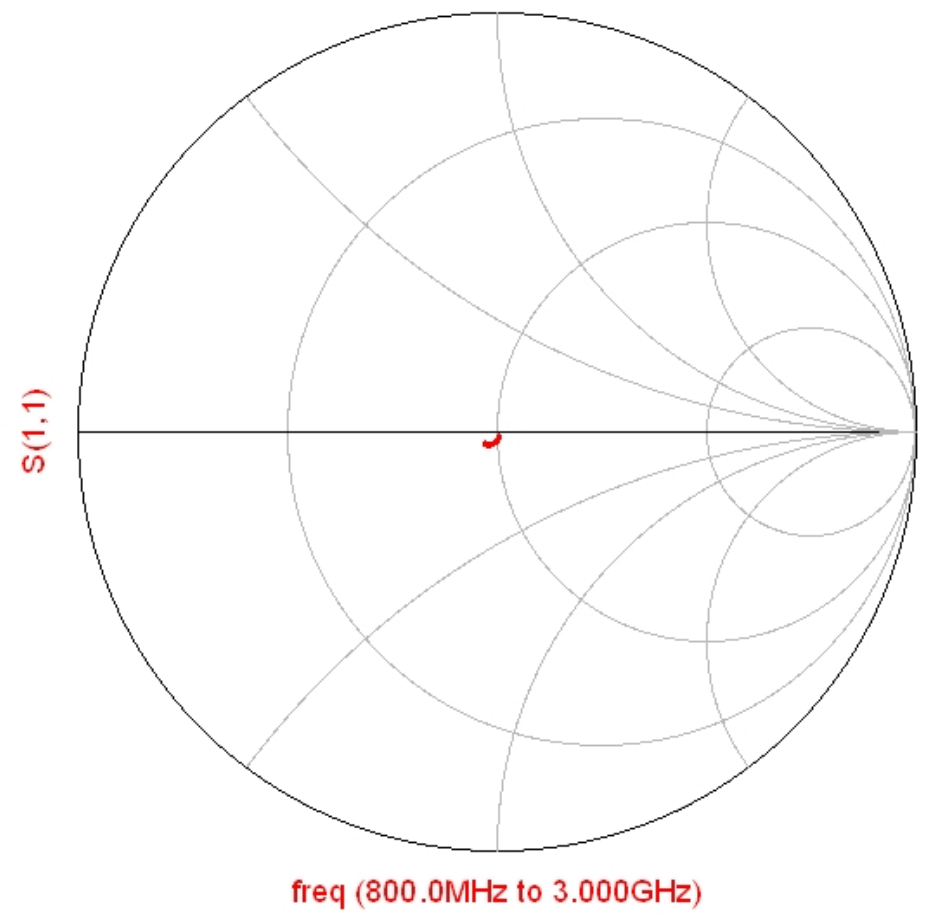

Figure 40. Simulation Output with Different Lengths of Thermal Reliefs.

As seen by Figures 39 and 40 and Table 13, the length of the stub does not affect the impedance of the circuit even when the stub length exceeded one-tenth of a wavelength. 
Table 13. $S_{11}$ and Impedance for Different Lengths of Thermal Reliefs.

\begin{tabular}{|c|c|c|}
\hline Length & $S_{11}$ & Impedance \\
\hline $1 \mathrm{~mm}$ & $0.039 \angle-129.3$ & $47.5-\mathrm{j} 2.9$ \\
\hline $2 \mathrm{~mm}$ & $0.038 \angle-130$ & $47.6-\mathrm{j} 2.8$ \\
\hline $3 \mathrm{~mm}$ & $0.037 \angle-132.3$ & $47.5-\mathrm{j} 2.7$ \\
\hline $4 \mathrm{~mm}$ & $0.040 \angle-130.2$ & $47.4-\mathrm{j} 2.9$ \\
\hline $5 \mathrm{~mm}$ & $0.039 \angle-129.9$ & $47.5-\mathrm{j} 2.8$ \\
\hline $6 \mathrm{~mm}$ & $0.038 \angle-130.9$ & $47.5-\mathrm{j} 2.8$ \\
\hline $7 \mathrm{~mm}$ & $0.039 \angle-132.2$ & $47.4-\mathrm{j} 2.8$ \\
\hline $8 \mathrm{~mm}$ & $0.039 \angle-130.3$ & $47.5-\mathrm{j} 2.9$ \\
\hline $9 \mathrm{~mm}$ & $0.038 \angle-130.5$ & $47.5-\mathrm{j} 2.8$ \\
\hline $10 \mathrm{~mm}$ & $0.038 \angle-131.4$ & $47.5-\mathrm{j} 2.8$ \\
\hline
\end{tabular}

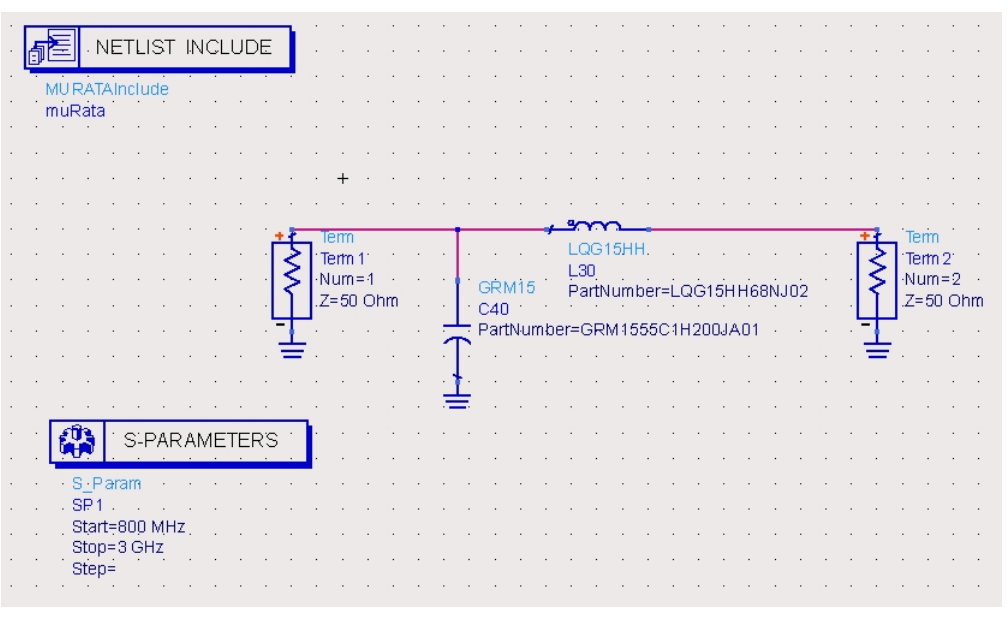

Figure 41. DC Bias Network Schematic

\subsection{Bias Networks}

The varactors and switch need to have a DC bias applied to them. However, it is important that no RF signals get into the DC bias networks as this will cause problems with the circuit operation. The standard bias network of a shunt capacitor and a series inductor was simulated.

From the Figure 42, the insertion loss at $920 \mathrm{MHz}$ is $-36.4 \mathrm{~dB}$ and at $2450 \mathrm{MHz}$ it is $-36 \mathrm{~dB}$. This is more then adequate to isolate the RF from the DC bias supply. 


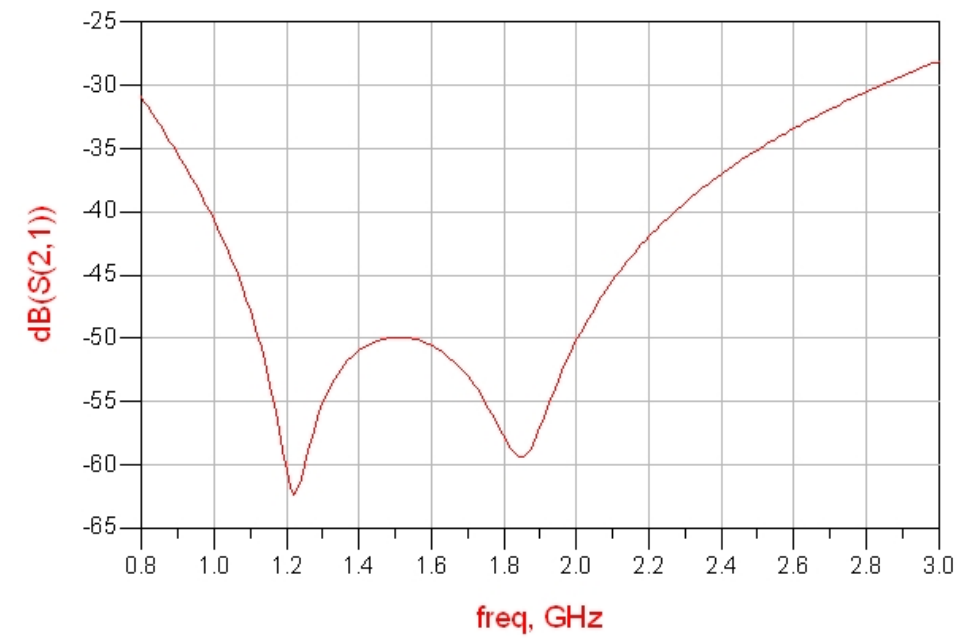

Figure 42. Insertion Loss of the DC Bias Network.

\subsection{Fabrication}

Now that all of the issues with the layout have been resolved, the next step is to actually layout the circuit and build it. ADS' layout editor was used to create the layout. The board was manufactured using the LPKF milling machine. The vias were filled using a copper rivet that was supplied with the LPKF milling machine. The final layout and the assembled pcb are shown in Figures 43 and 44 .

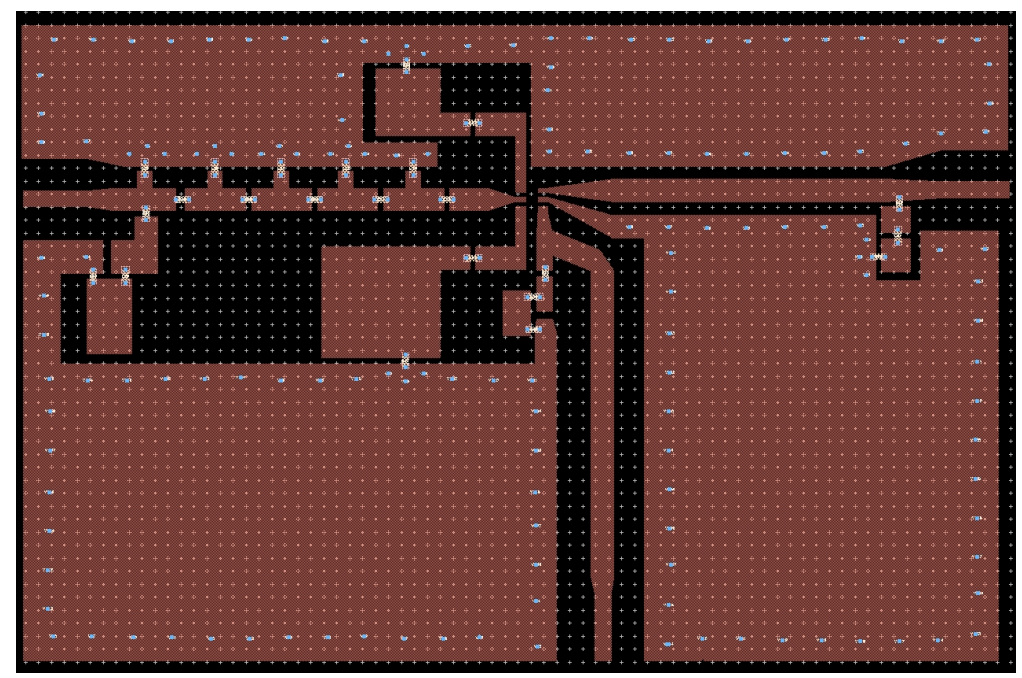

Figure 43. Final Layout for the Printed Circuit Board. 


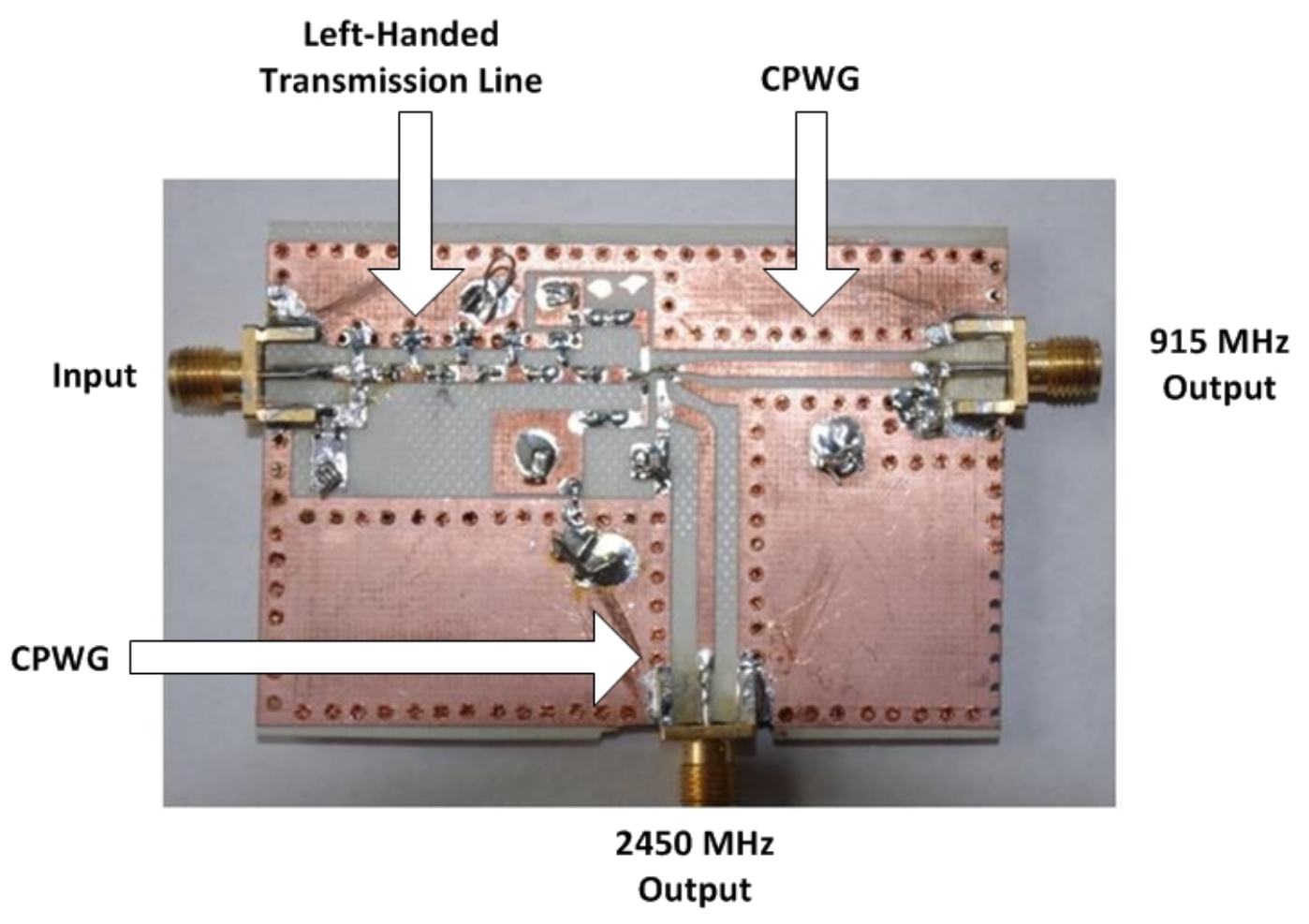

Figure 44. Assembled Printed Circuit Board.

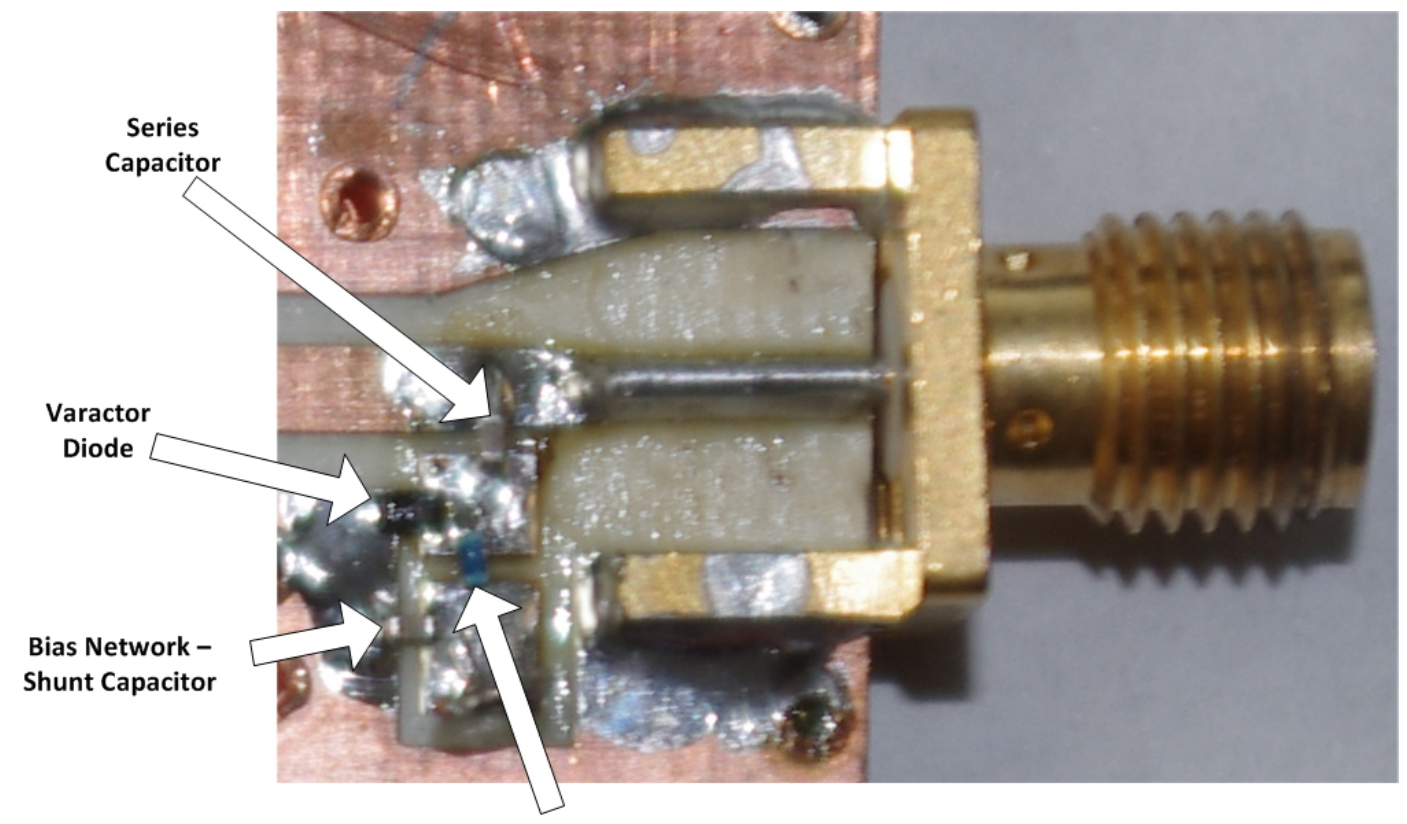

Bias Network -

Series Inductor

Figure 45. Close Up of the Varactor Diode and Bias Network. 


\section{CHAPTER 6. TESTING}

The original intent of the phase shifter was to have two output ports: $915 \mathrm{MHz}$ and $2450 \mathrm{MHz}$. This would enable the CPWG of either output to be modified if it was neccessary. However, due to switch failure, the $915 \mathrm{MHz}$ port was used for both frequency bands.

\subsection{Equipment Used}

The Agilent E5071C network analyzer was used to measure the phase shift (angle of $S_{21}$ ), insertion loss (magnitude of $S_{21}$ ), and return loss (magnitude of $S_{11}$ ). A RSR HY3002-3 power supply was used for the DC source for biasing the varactors and switch.

\subsection{Testing and Results}

The board was connected to the network analyzer and DC bias was applied. Figures 46 and 47 are screen captures from the network analyzer with the varactor diode DC bias set at $1 \mathrm{~V}$. The phase shift for each bias voltage is shown in Table 14 . The insertion loss, and return loss for each bias voltages is shown in Table 15 . 


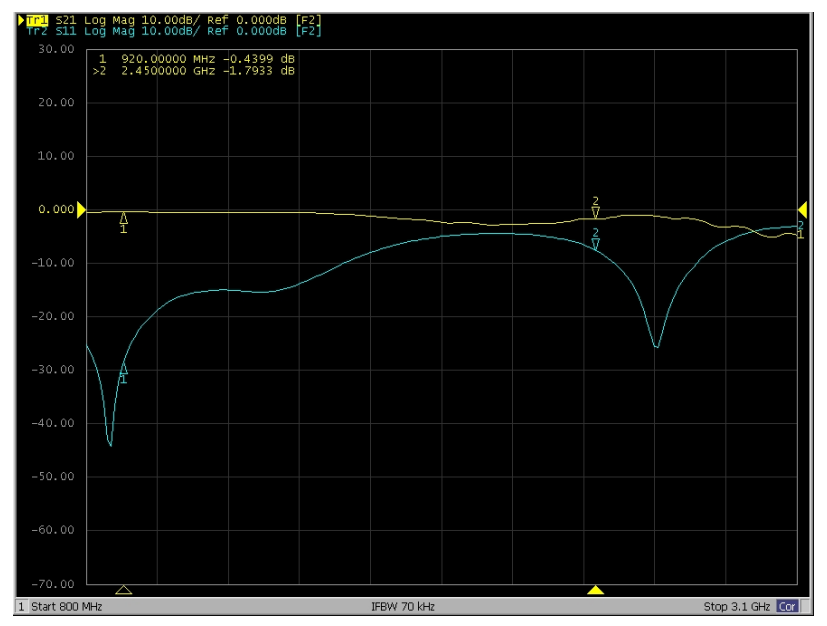

Figure 46. Magnitude of $S_{11}$ and $S_{21}$ of the Phase Shifter with the DC Bias for the Varactor Diodes Set at 1 Volt.

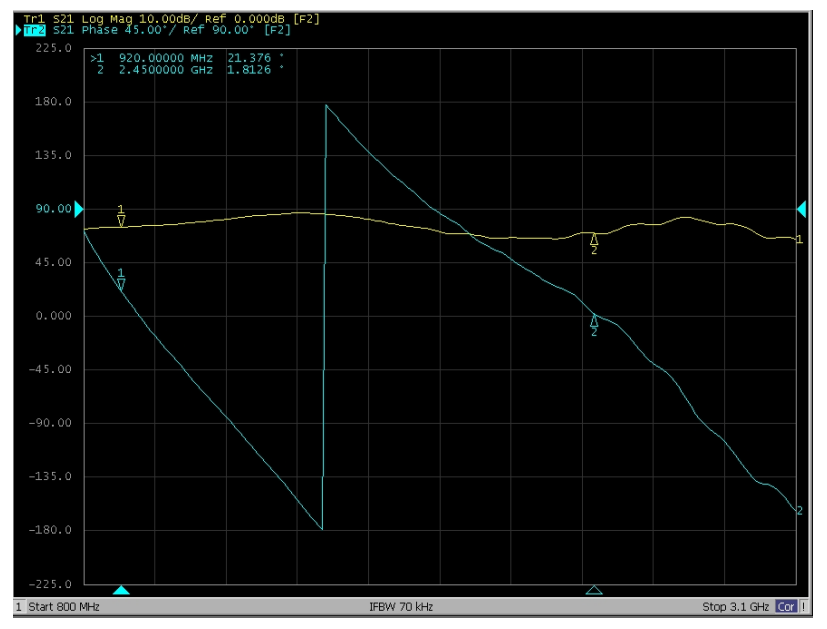

Figure 47. Phase Shift and Magnitude of $S_{21}$ of the Phase Shifter with the DC Bias for the Varactor Diodes Set at 1 Volt. 
Table 14. Phase Shift for each Bias Voltage with a 1-pF Series Capacitor.

\begin{tabular}{|c|c|c|}
\hline Bias Voltage & $S_{21}$ Phase at $920 \mathrm{MHz}$ & $S_{21}$ Phase at $2450 \mathrm{MHz}$ \\
\hline 0 & 17 & -2.6 \\
\hline 0.1 & 17.2 & -2.3 \\
\hline 0.2 & 17.5 & -1.9 \\
\hline 0.3 & 17.8 & -1.5 \\
\hline 0.4 & 18 & -1.3 \\
\hline 0.5 & 20.3 & 0.74 \\
\hline 0.6 & 20.6 & 1.1 \\
\hline 0.7 & 20.7 & 1.2 \\
\hline 0.8 & 21 & 1.6 \\
\hline 0.9 & 21.2 & 1.6 \\
\hline 1 & 21.4 & 1.8 \\
\hline 1.5 & 22.1 & 2.6 \\
\hline 2 & 22.8 & 3.2 \\
\hline 2.5 & 23.4 & 3.9 \\
\hline 3 & 24 & 4.4 \\
\hline 4 & 25.2 & 5.5 \\
\hline 5 & 26.4 & 6.4 \\
\hline 6 & 27 & 7 \\
\hline 7 & 27.1 & 7 \\
\hline
\end{tabular}


Table 15. Insertion and Return Loss for each Bias Voltage with a 1-pF Series Capacitor.

\begin{tabular}{|c|c|c|c|c|}
\hline $\begin{array}{c}\text { Bias } \\
\text { Voltage }\end{array}$ & $\begin{array}{c}\text { Insertion Loss } \\
\text { at 920 MHz }\end{array}$ & $\begin{array}{c}\text { Return Loss } \\
\text { at 920 MHz }\end{array}$ & $\begin{array}{c}\text { Insertion Loss } \\
\text { at 2450 MHz }\end{array}$ & $\begin{array}{c}\text { Return Loss } \\
\text { at 2450 MHz }\end{array}$ \\
\hline 0 & 0.44 & 22 & 1.6 & 8 \\
\hline 0.1 & 0.35 & 28 & 1.6 & 8 \\
\hline 0.2 & 0.35 & 26 & 1.6 & 8 \\
\hline 0.3 & 0.35 & 25 & 1.6 & 8 \\
\hline 0.4 & 0.33 & 32 & 1.5 & 8 \\
\hline 0.5 & 0.4 & 30 & 1.6 & 8 \\
\hline 0.6 & 0.49 & 28 & 1.8 & 7 \\
\hline 0.7 & 0.45 & 28 & 1.8 & 8 \\
\hline 0.8 & 0.47 & 29 & 1.8 & 7 \\
\hline 0.9 & 0.45 & 28 & 1.8 & 7 \\
\hline 1 & 0.44 & 28 & 1.8 & 7 \\
\hline 1.5 & 0.44 & 29 & 1.8 & 7 \\
\hline 2 & 0.45 & 25 & 1.8 & 7 \\
\hline 2.5 & 0.45 & 25 & 1.8 & 7 \\
\hline 3 & 0.45 & 25 & 1.8 & 7 \\
\hline 4 & 0.45 & 25 & 1.8 & 7.8 \\
\hline 5 & 0.45 & 25 & 1.8 & 7.8 \\
\hline 6 & 0.45 & 25 & & 7 \\
\hline 7 & 0.45 & 25 & & \\
\hline
\end{tabular}

The total phase shift (phase shift at $7 \mathrm{~V}$ minus the phase shift at $0 \mathrm{~V}$ ) with the $1-\mathrm{pF}$ series capacitor was $10.1^{\circ}$ at $920 \mathrm{MHz}$ and $9.6^{\circ}$ at $2450 \mathrm{MHz}$. This phase shift is not sufficient. In examining the circuit, the 1-pF capacitor in series with the varactor diode was replaced with a 10-pF capacitor. The phase shift for each bias voltage is shown in Table 16. The insertion loss, and return loss for each bias voltages is shown in Table 17. 
Table 16. Phase Shift for each Bias Voltage with a 10-pF Series Capacitor.

\begin{tabular}{|c|c|c|}
\hline $\begin{array}{c}\text { Bias } \\
\text { Voltage }\end{array}$ & $\begin{array}{c}S_{21} \text { Phase } \\
\text { at } 920 \mathrm{MHz}\end{array}$ & $\begin{array}{c}S_{21} \text { Phase } \\
\text { at } 2450 \mathrm{MHz}\end{array}$ \\
\hline 0 & -51 & 65 \\
\hline 0.1 & -50.8 & 53.7 \\
\hline 0.2 & -49 & 36 \\
\hline 0.3 & -47.7 & 18 \\
\hline 0.4 & -45.6 & 1.6 \\
\hline 0.5 & -44 & -10.8 \\
\hline 0.6 & -43.6 & -25.8 \\
\hline 0.7 & -43 & -70 \\
\hline 0.8 & -42 & -90 \\
\hline 0.9 & -41.5 & -107 \\
\hline 1 & -41 & -133 \\
\hline 1.5 & -38 & -148 \\
\hline 2 & -35 & -143 \\
\hline 2.5 & -34 & -133 \\
\hline 3 & -32 & -119 \\
\hline 4 & -29 & -88 \\
\hline 5 & -26 & -63 \\
\hline 6 & -25 & -53 \\
\hline 7 & -25 & -50 \\
\hline
\end{tabular}

With the $10 \mathrm{pF}$ series capacitor, the total phase shift (phase shift at $7 \mathrm{~V}$ minus the phase shift at $0 \mathrm{~V}$ ) was $26^{\circ}$ at $920 \mathrm{MHz}$ and $115^{\circ}$ at $2450 \mathrm{MHz}$. These results were satisfactory. 
Table 17. Insertion and Return Loss for each Bias Voltage with a 10-pF Series Capacitor.

\begin{tabular}{|c|c|c|c|c|}
\hline $\begin{array}{c}\text { Bias } \\
\text { Voltage }\end{array}$ & $\begin{array}{c}\text { Insertion Loss } \\
\text { at } 920 \mathrm{MHz}\end{array}$ & $\begin{array}{l}\text { Return Loss } \\
\text { at } 920 \mathrm{MHz}\end{array}$ & $\begin{array}{l}\text { Insertion Loss } \\
\text { at } 2450 \mathrm{MHz}\end{array}$ & $\begin{array}{l}\text { Return Loss } \\
\text { at } 2450 \mathrm{MHz}\end{array}$ \\
\hline 0 & 0.44 & 22 & 2 & 8 \\
\hline 0.1 & 0.35 & 28 & 1.6 & 8 \\
\hline 0.2 & 0.35 & 26 & 1.6 & 8 \\
\hline 0.3 & 0.35 & 25 & 1.6 & 8 \\
\hline 0.4 & 0.33 & 32 & 1.5 & 8 \\
\hline 0.5 & 0.4 & 30 & 1.6 & 8 \\
\hline 0.6 & 0.49 & 28 & 1.5 & 7 \\
\hline 0.7 & 0.45 & 28 & 1.8 & 8 \\
\hline 0.8 & 0.47 & 29 & 1.8 & 7 \\
\hline 0.9 & 0.45 & 28 & 1.8 & 7 \\
\hline 1 & 0.44 & 28 & 1.8 & 7 \\
\hline 1.5 & 0.44 & 29 & 1.8 & 7 \\
\hline 2 & 0.45 & 25 & 1.8 & 7 \\
\hline 2.5 & 0.45 & 25 & 1.8 & 7 \\
\hline 3 & 0.45 & 25 & 1.8 & 7 \\
\hline 4 & 0.45 & 25 & 1.8 & 7 \\
\hline 5 & 0.45 & 25 & 1.8 & 7 \\
\hline 6 & 0.45 & 25 & 1.8 & 7 \\
\hline 7 & 0.45 & 25 & 1.8 & 7 \\
\hline
\end{tabular}




\subsection{Overall Performance}

In Chapter 2, a list of of parameters for the performance of a phase shifter was presented. The performance of the prototype is evaluated for each criteria.

\subsubsection{Phase Shift Range}

The total phase shift for both the $920-\mathrm{MHz}$ and the $2450-\mathrm{MHz}$ band was good. The fact that all of the phase shifts for the $920-\mathrm{MHz}$ band were negative (instead of positive) can be resolved by adjusting the length of the right-handed transmission line. The phase shifts for the $2450-\mathrm{MHz}$ band were excellent since they could be adjusted from negative to positive.

\subsubsection{Phase Shift Increment}

For both bands the increment was very good. The step size for the $920 \mathrm{MHz}$ band is smaller than the step size for the $2450-\mathrm{MHz}$ band, but that was expected. The increment size would be decreased (for both bands) if a smaller voltage step could be obtained.

\subsubsection{Frequency and Bandwidth of Operation}

The frequency and bandwidth of operation were excellent since the phase shifter was able to function across the entire frequency range for both bands.

\subsubsection{Physical Size}

Size was not a major concern for this design (except that each unit cell of the

left-handed transmission line needed to be less then or equal to $\frac{\lambda}{10}$. However the size was such that it would function very well in a cellular telephone base station.

\subsubsection{Insertion Loss}

The desired value for the insertion loss was $0.2 \mathrm{~dB}$ or less. This insertion loss would result in only $4.5 \%$ of the input power that would be dissipated in the circuit. The insertion loss at $920 \mathrm{MHz}$ was good since it was only slightly greater (0.45 dB) then the target value. This would result in $10 \%$ of the input power being dissipated 
in the circuit. At $2450 \mathrm{MHz}$ the insertion loss was $2 \mathrm{~dB}$, an acceptable value. The 2 $\mathrm{dB}$ insertion loss would mean that $37 \%$ of the input power is dissipated in the circuit. Refer to Appendix I for the details on the target values and analysis of the insertion loss.

\subsubsection{Return Loss (or Impedance Matching)}

The target value for the return loss was $20 \mathrm{~dB}$ or greater. The return loss for the $920 \mathrm{MHz}$ band was excellent since it exceeded the target value by 2 to $8 \mathrm{~dB}$ depending on the bias voltage. The return loss at $2450 \mathrm{MHz}$ was fair because it was $12 \mathrm{~dB}$ below the target value. This return loss would result in $16 \%$ of the power being reflected back to the source. Appendix I has the details on the target values and analysis of the return loss. 


\section{CHAPTER 7. CONCLUSION}

The performance of the phase shifter was good to excellent. The design procedure is easy to follow and the results are close to the optimized component values that were obtained from the simulation program.

The first step to improve the return loss for the $2450 \mathrm{MHz}$ band would be to get a professionally manufactured printed circuit board. One of the main issues with the test board was that copper rivets were used for the plating of the vias. Some of the problems with using these copper rivets are:

1. The hand insertion method does not permit repeatability, i.e. each rivet is different.

2. The material used for these rivets is not optimized for high frequency work.

3. The electrical connection to the printed circuit board will vary depending on the location of the rivet and the quality of the solder joints.

The second issue that would require additional investigation is the SMA launch to the printed circuit board. When the signal goes from the SMA connector, there can be a very large discontinuity. Another issue is the transition from a coaxial structure to a microstrip transmission line. Also since the SMA connector has a round center conductor that is soldered onto the $\mathrm{PCB}$, this physical discontinuity can cause an electrical discontinuity. Finally, the grounding of the PCB will affect the performance of this launch. Ideally a time-domain reflectometer (TDR) would be used to analyze this discontinuity and, based on this measurement, the launch would be improved. 


\section{REFERENCES}

[1] Arnab Bhattacharya (2013, September 26). "Modeling and Simulation of Metamaterial-Based Devices for Industrial Applications Webinar," Computer Simulation Technology, Available: http://www.cst.com

[2] C. Caloz and T. Itoh, "Application of the Transmission Line Theory of LeftHanded Materials to the Realization of a Microstrip LH Line," IEEE Transactions on Antennas and Propagation, International Symposium Digest, Vol. 2, pp.412-415, June 2002.

[3] B. Braaten and R. Scheeler, "Radio Frequency Identification Fundamentals and Applications, Design Methods and Solutions," Intech, February, 2010, Chapter 4 .

[4] David M. Pozar, Microwave Engineering, $3^{\text {rd }}$ Ed., John Wiley and Sons, Inc., New York, NY, August 2005, p. 185.

[5] Erwin Kreyszig, Advanced Engineering Mathematics, John Wiley and Sons, Inc., New York, NY, 1999, p. 348.

[6] C. Sabah, F. Urbani, and S. Uckun, "Bloch Impedance Analysis for a LeftHanded Transmission Line," Journal of Electrical Engineering, Vol. 63, No. 5, pp.310-315, 2012.

[7] C. Caloz and T Itoh, "Novel Microwave Devices and Structures Based on the Transmission Line Approach to Meta-Materials," IEEE Microwave Theory and Techniques Symposium Digest, pp.195-198, June 2003.

[8] R.V. Garver, "Broad-Band Diode Phase Shifters," IEEE Transactions on Microwave Theory and Techniques, Vol. 22, pp.314-323, May 1972. 
[9] J.F. White, "High Power, p-i-n Controlled, Microwave Transmission Phase Shifters," IEEE Transactions on Microwave Theory and Techniques, Vol. 13, pp.233-243, May 1965.

[10] T. Yahara, "A Note on Designing Digital Loaded Line Phase Shifters," IEEE Transactions on Microwave Theory and Techniques, Vol. 20, pp.703-704, October 1972.

[11] H.A. Atwate, "Circuit Design of the Loaded-Line Phase Shifter," IEEE Transactions on Microwave Theory and Techniques, Vol. 33, pp.626-634, July 1985.

[12] F.L. Opp and W.F. Hoffman, "Design of Digital Loaded Line Phase Shift Networks for Microwave Thin Film Applications," IEEE Transactions on Microwave Theory and Techniques, Vol. 16, pp.426-468, July 1968.

[13] W.A. Davis, "Design Equations and Bandwidth of Loaded-Line Phase Shifters," IEEE Transactions on Microwave Theory and Techniques, Vol. 22, pp.561-563, May 1974.

[14] P. He, J. Gao, C.T. Marinis, P.V. Parimi, C. Virrotia, and V.G. Harris, "A Microstrip Tunable Negative Refractive Index Metamaterial and Phase Shifter," Applied Physics Letters, Vol. 93, November 2008.

[15] Hongjoon Kim, A. Kozyrev, A. Karbassi, and D.W. van der Weide, "Linear Tunable Phase Shifter Using a Left-Handed Transmission Line," IEEE Microwave and Wireless Components Letters, Vol. 15, pp. 366-368, May 2000.

[16] D. Kyulenstierna, A. Vorobiev, P. Linner, and S. Gevorgian, "Composite Right/Left Handed Transmission Line Phase Shifter Using Ferroelectric Varactors," IEEE Microwave and Wireless Components Letters, Vol. 16, pp. 167-169, April 2006. 
[17] M. Antoniades and G. Eleftheriades, "Compact Linear Lead/Lag Metamaterial Phase Shifters for Broadband Applications," IEEE Antennas and Wireless Propagation Letters, Vol. 2, pp. 103-106, May 2003.

[18] A. Giere, C. Damm, P. Scheele, and R. Jakoby, "LH Phase Shifter using Ferroelectric Varactors," IEEE Radio and Wireless Symposium, pp. 403-406, October 2006.

[19] IH. Lin, M. DeVincentis, C. Caloz, and T. Itoh, "Arbitrary Dual-Band Components Using Composite Right/Left Handed Transmission Lines," IEEE Transactions on Microwave Theory and Techniques, Vol. 52, No. 4, pp. 1142-1149, April 2004 .

[20] University of South Florida, "Numerical Methods for STEM Undergraduate," Available: http://www.youtube.com/watch?v=NByHuFBkulw.

[21] K. Blattenberger, "Capacitors \& Capacitance Calculations," Available: http://www.rfcafe.com/references/electrical/capacitance.htm.

[22] K. Blattenberger, "Inductors \& Inductance Calculations," Available: http://www.rfcafe.com/references/electrical/inductance.htm. 


\section{APPENDIX A. DERIVATION FOR THE ABCD MATRIX FOR THE D/2 TRANSMISSION LINE}

In this appendix the $\mathrm{ABCD}$ matrix is derived from the $\mathrm{ABCD}$ matrix for an arbitrary length transmission line. The ABCD matrix for a transmission line is:

$$
\left[\begin{array}{cc}
\cos \beta l & j Z_{0} \sin \beta l \\
j Y_{0} \sin \beta l & \cos \beta l,
\end{array}\right]
$$

where

$$
\beta=\frac{\omega}{v_{p}}
$$

and

$$
l=\frac{d}{2} .
$$

Multiplying $\beta$ and $l$ together yields

$$
\beta l=\left(\frac{\omega}{v_{p}}\right)\left(\frac{d}{2}\right) \Rightarrow \frac{\left(\frac{\text { radians }}{\text { sec }}\right)}{\left(\frac{\text { meters }}{\text { sec }}\right)} \text { meters }=\text { radians } .
$$

This then yields

$$
\beta l=\frac{\theta}{2} .
$$




\section{APPENDIX B. ABCD MATRIX SIMPLIFICATION FOR THE PERIODICALLY LOADED INFINITE TRANSMISSION LINE.}

From (1.10),

$$
\left[\begin{array}{ll}
A & B \\
C & D
\end{array}\right]=\left[\begin{array}{cc}
\cos \frac{\theta}{2} & j \sin \frac{\theta}{2} \\
j \sin \frac{\theta}{2} & \cos \frac{\theta}{2}
\end{array}\right]\left[\begin{array}{cc}
1 & 0 \\
j b & 1
\end{array}\right]\left[\begin{array}{cc}
\cos \frac{\theta}{2} & j \sin \frac{\theta}{2} \\
j \sin \frac{\theta}{2} & \cos \frac{\theta}{2}
\end{array}\right]
$$

The product of the first two matricies is

$$
\left[\begin{array}{cc}
\cos \frac{\theta}{2} & j \sin \frac{\theta}{2} \\
j \sin \frac{\theta}{2} & \cos \frac{\theta}{2}
\end{array}\right]\left[\begin{array}{ll}
1 & 0 \\
j b & 1
\end{array}\right]=\left[\begin{array}{cc}
\cos \frac{\theta}{2}-b \sin \frac{\theta}{2} & j \sin \frac{\theta}{2} \\
j \sin \frac{\theta}{2}+j b \cos \frac{\theta}{2} & \cos \frac{\theta}{2}
\end{array}\right],
$$

which results in

$$
\left[\begin{array}{cc}
\cos \frac{\theta}{2}-b \sin \frac{\theta}{2} & j \sin \frac{\theta}{2} \\
j \sin \frac{\theta}{2}+j b \cos \frac{\theta}{2} & \cos \frac{\theta}{2}
\end{array}\right]\left[\begin{array}{cc}
\cos \frac{\theta}{2} & j \sin \frac{\theta}{2} \\
j \sin \frac{\theta}{2} & \cos \frac{\theta}{2}
\end{array}\right]
$$

This becomes

$$
\left[\begin{array}{cc}
\cos ^{2} \frac{\theta}{2}-b \cos \frac{\theta}{2} \sin \frac{\theta}{2}-\sin ^{2} \frac{\theta}{2} & j \sin \frac{\theta}{2} \cos \frac{\theta}{2}-j b \sin ^{2} \frac{\theta}{2}+j \sin \frac{\theta}{2} \cos \frac{\theta}{2} \\
j \sin \frac{\theta}{2} \cos \frac{\theta}{2}+j b \cos ^{2} \frac{\theta}{2}+j \sin \frac{\theta}{2} \cos \frac{\theta}{2} & \cos ^{2} \frac{\theta}{2}-b \cos \frac{\theta}{2} \sin \frac{\theta}{2}-\sin ^{2} \frac{\theta}{2}
\end{array}\right]
$$

Using the following identities, the matrix is simplified:

$$
\begin{aligned}
& \cos ^{2} a-\sin ^{2} a=\cos 2 a \\
& \sin ^{2} a=\frac{1}{2}(1-\cos 2 a)
\end{aligned}
$$




$$
\begin{gathered}
\cos ^{2} a=\frac{1}{2}(1+\cos 2 a \\
\sin a \cos a=\frac{1}{2}(\sin (a+b)+\sin (a-b)
\end{gathered}
$$

Thus (B.1) becomes

$$
\left[\begin{array}{cc}
\cos \theta-\frac{b}{2} \sin \theta & j\left(\sin \theta-\frac{b}{2}+\frac{b}{2} \cos \theta\right) \\
j\left(\sin \theta+\frac{b}{2}+\frac{b}{2} \cos \theta\right) & \cos \theta-\frac{b}{2} \sin \theta
\end{array}\right] .
$$




\section{APPENDIX C. ANALYSIS OF A RECIPROCAL NETWORK}

A symmetrical network is defined by $\mathrm{AD}-\mathrm{BC}=1$. Here,

$$
\left[\begin{array}{ll}
A & B \\
C & D
\end{array}\right]=\left[\begin{array}{cc}
\cos \theta-\frac{b}{2} \sin \theta & j\left(\sin \theta+\frac{b}{2} \cos \theta-\frac{b}{2}\right) \\
j\left(\sin \theta+\frac{b}{2} \cos \theta+\frac{b}{2}\right) & \cos \theta-\frac{b}{2} \sin \theta,
\end{array}\right]
$$

so that

$$
A D=\cos \theta^{2}-b \sin \theta \cos \theta+\frac{b^{2}}{4} \sin \theta^{2}
$$

and

$$
B C=-\sin \theta^{2}-b \sin \theta \cos \theta-\frac{b^{2}}{4} \cos \theta^{2}+\frac{b^{2}}{4}
$$

Therefore,

$A D-B C=\left(\cos \theta^{2}-b \sin \theta \cos \theta+\frac{b^{2}}{4} \sin \theta^{2}\right)-\left(-\sin \theta^{2}-b \sin \theta \cos \theta-\frac{b^{2}}{4} \cos \theta^{2}+\frac{b^{2}}{4}\right)$

and

$$
A D-B C=\cos \theta^{2}+\sin \theta^{2}=1 \text {. }
$$




\section{APPENDIX D. TRUNCATION ERROR FOR COS $\theta$}

The MacLauren Series for the cosine function is

$$
\cos (x)=1-\frac{x^{2}}{2 !}+\frac{x^{4}}{4 !}+\ldots
$$

If the series is trucated after the third term, what is the error compared to using more terms? The method presented by USF [20] was used to determine the error. The following example with $\mathrm{x}=60$ degrees is used to illustrate this method.

Table D.1. Error versus Number of Terms.

\begin{tabular}{|c|c|c|c|}
\hline Number of Terms & $\cos (\mathrm{x})$ & Approximate Error & Absolute Relative Error (\%) \\
\hline 1 & 1 & - & - \\
\hline 2 & 0.4517 & 0.0483 & 10.6957 \\
\hline 3 & 0.5018 & 0.0501 & 9.9856 \\
\hline
\end{tabular}

The resulting truncation error is less then $10 \%$. 


\section{APPENDIX E. CASE 1: $\alpha=0, \beta \neq 0$ AND CASE 2: $\alpha \neq 0$, $\beta=0$ DERIVATIONS}

Two expressions for $\cosh (\gamma d)$ are used in Chapter 1.

Case 1: $\alpha=0$ and $\beta \neq 0$

$$
\cosh \gamma d=\cosh (0 d) \cos (\beta d)+j \sinh (0 d) \sin (\beta d)
$$

or

$$
\cosh \gamma d=\cos \beta d
$$

Case 2: $\alpha \neq 0$ and $\beta=0$

$$
\cosh \gamma d=\cosh (\alpha d) \cos (0 d)+j \sinh (\alpha d) \sin (0 d)
$$

or

$$
\cosh \gamma d=\cosh \alpha d
$$




\section{APPENDIX F. BLOCH IMPEDANCE DERIVATION}

Equation (1.46) is subtituted into (1.28), shown here for ease of reading.

$$
Z_{B}=\frac{-Z_{0} B}{A-e^{\gamma d}}
$$

and

$$
e^{\gamma d}=\frac{(A+D) \pm \sqrt{(A+D)^{2}-4}}{2} .
$$

Thus,

$$
Z_{B}=\frac{-Z_{0} B}{A-\frac{(A+D) \pm \sqrt{(A+D)^{2}-4}}{2}}
$$

Since this is a symmetrical circuit, $\mathrm{A}=\mathrm{D}$. Thus,

$$
Z_{B}=\frac{-Z_{0} B}{A-\frac{(2 A) \pm \sqrt{\left(4 A^{2}-4\right)}}{2}}=\frac{-Z_{0} B}{A-A \pm \sqrt{A^{2}-1}}
$$

or

$$
Z_{B}=\frac{-Z_{0} B}{ \pm \sqrt{A^{2}-1}}
$$

Substituting the values for A and B,

$$
Z_{B}=\frac{-Z_{0}\left(2 Z_{s}+\frac{Z_{s}^{2}}{Z_{p}}\right)}{ \pm \sqrt{\left(1+\frac{Z_{s}}{Z_{p}}\right)^{2}-1}}
$$

Simplifying the denominator first,

$$
\left(1+\frac{Z_{s}}{Z_{p}}\right)^{2}-1=\frac{Z_{s}^{2}}{Z_{p}^{2}}+2 \frac{Z_{s}}{Z_{p}}+1-1=\frac{Z_{s}^{2}}{Z_{p}^{2}}+2 \frac{Z_{s}}{Z_{p}}=\frac{Z_{s}^{2}+2 Z_{s} Z_{p}}{Z_{p}^{2}}
$$

Thus

$$
Z_{B}=-Z_{0}\left(\frac{2 Z_{s} Z_{p}+Z_{s}^{2}}{Z_{p}}\right) \sqrt{\frac{Z_{p}^{2}}{Z_{s}^{2}+2 Z_{s} Z_{p}}}
$$


Or

$$
Z_{B}=-Z_{0} \sqrt{2 Z_{s} Z_{p}+Z_{S}^{2}}=-Z_{0} \sqrt{Z_{s}\left(2 Z_{p}+Z_{s}\right)} .
$$




\section{APPENDIX G. ABCD MATRIX FOR A LOSSLESS LOADED-LINE PHASE SHIFTER.}

The ABCD matrix for a lossless loaded-line phase shifter is composed of three seperate ABCD matrcies [4]:

$$
\begin{aligned}
\text { shunt element } \Rightarrow\left[\begin{array}{cc}
1 & 0 \\
j b & 1
\end{array}\right], \\
\text { arbitrary length transmission line } \Rightarrow\left[\begin{array}{cc}
\cos \beta l & j Z_{c} \sin \beta l \\
j Y_{c} \sin \beta l & \cos \beta l
\end{array}\right],
\end{aligned}
$$

and

$$
\text { shunt element } \Rightarrow\left[\begin{array}{cc}
1 & 0 \\
j b & 1
\end{array}\right]
$$

This then gives

$$
\left[\begin{array}{ll}
A & B \\
C & D
\end{array}\right]=\left[\begin{array}{cc}
1 & 0 \\
j b & 1
\end{array}\right]\left[\begin{array}{cc}
\cos \beta l & j \sin \beta l \\
j \sin \beta l & \cos \beta l
\end{array}\right]\left[\begin{array}{cc}
1 & 0 \\
j b & 1
\end{array}\right] .
$$

Combining the first two matrices yields

$$
\left[\begin{array}{cc}
\cos \beta l & j Z_{c} \sin \beta l \\
j b \cos \beta l+j Y_{c} \sin \beta l & -b Z_{c} \sin \beta l+\cos \beta l
\end{array}\right]\left[\begin{array}{cc}
1 & 0 \\
j b & 1
\end{array}\right]
$$

which results in the final matrix

$$
\left[\begin{array}{cc}
\cos \beta l-b Z_{c} \sin \beta l & j Z_{c} \sin \beta l \\
j Z_{c}\left(2 b Y_{c} \cos \beta l+\left(Y_{c}^{2}-b^{2}\right) \cos \beta l\right) & \cos \beta l-b Z_{c} \sin \beta l
\end{array}\right] .
$$


Subtituting $B_{L 1}=b$ in (G.6) gives the matrix used,

$$
\left[\begin{array}{cc}
\cos \beta l-B_{L 1} Z_{c} \sin \beta l & j Z_{c} \sin \beta l \\
j Z_{c}\left(2 B_{L 1} Y_{c} \cos \beta l+\left(Y_{c}^{2}-B_{L 1}^{2}\right) \cos \beta l\right) & \cos \beta l-B_{L 1} Z_{c} \sin \beta l
\end{array}\right] .
$$




\section{APPENDIX H. DERIVING THE MAGNITUDE AND ANGLE OF $S_{21}$ FOR A LOADED LINE PHASE SHIFTER.}

From Chapter 2, it is known that

$$
S_{21}=\frac{2}{A+\frac{B}{Z_{0}}+C Z_{0}+D}
$$

Substituting (2.5) through (2.8) into (H.1) yields:

$$
S_{21}=\frac{\cos \beta l-B_{L 1} Z_{c} \sin \beta l-j Z_{c} Y_{0} \sin \beta l}{\cos \beta l-B_{L 1} Z_{c} \sin \beta l+j Z_{c} Y_{0} \sin \beta l\left[\cos \beta l-B_{L 1} Z_{c} \sin \beta l-j Z_{c} Y_{0} \sin \beta l\right]}
$$

and the magnitude of $S_{21}$ is given by

$$
\left|S_{21}\right|=\sqrt{\frac{1}{\left(\cos \beta l-B_{L 1} Z_{c} \sin \beta l\right)^{2}+\left(B_{L 1} Z_{0} \cos \beta l+\frac{Z_{c} Z_{0}}{2}\left(Y_{0}^{2}+Y_{c}^{2}-B_{L 1}^{2}\right) \sin \beta l\right)^{2}}} .
$$

The input is matched; therefore, $S_{11}=0$ and from (2.9),

$$
S_{11}=0=\frac{B Y_{0}-C Z_{0}}{2 A+B Y_{0}+C Z_{0}}
$$

and

$$
B Y_{0}=C Z_{0}
$$

Substituting B and C from (2.6) and (2.7) gives the condition for a matched input:

$$
Y_{0} Z_{c} \sin \beta l=Z_{0} Z_{c}\left(2 B_{L 1} Y_{c} \cos \beta l+\left(Y_{c}^{2}-B_{L 1}^{2}\right) \sin \beta l\right)
$$

The circuit is reciprocal, $\mathrm{A}=\mathrm{D}$, which then gives $S_{21}$ as

$$
S_{21}=\frac{2}{2 A+B Y_{0}+C Z_{0}}
$$


Using the matched condition $B Y_{0}=C Z_{0}$

$$
S_{21}=\frac{1}{A+B Y_{0}}
$$

Substitute (2.5) and (2.6) into (H.8)

$$
\begin{gathered}
S_{21}=\frac{1}{\left(\cos \beta l-B_{L 1} Z_{c} \sin \beta l\right)+j\left(Z_{c} Y_{0} \sin \beta l\right)}, \\
S_{21}=\frac{\left(\cos \beta l-B_{L 1} Z_{c} \sin \beta l\right)-j\left(Z_{c} Y_{0} \sin / \text { betal }\right)}{\left(\cos \beta l-B_{L 1} Z_{c} \sin \beta l\right)^{2}+\left(Z_{c} Y_{0} \sin \beta l\right)^{2}}, \\
\left|S_{21}\right|=\sqrt{\frac{1}{\left(\cos \beta l-B_{L 1} Z_{c} \sin \beta l\right)^{2}}+\left(Z_{c} Y_{0} \sin \beta l\right)^{2}}, \\
\angle S_{21}=\arccos \left(\cos \beta l-B_{L 1} Z_{c} \sin \beta l\right),
\end{gathered}
$$

which simplifies to:

$$
\angle S_{21}=\arcsin \left(-Z_{c} Y_{0} \sin \beta l\right)
$$

Note: (H.2) and (H.3) reduce to (H.10) and (H.11) when dealing with a matched, reciprocal network. 


\section{APPENDIX I. CALCULATION OF THE DESIRED GOALS FOR THE INSERTION LOSS AND RETURN LOSS}

\section{I.1. Desired Insertion Loss}

The desired insertion loss is $0.2 \mathrm{~dB}$, which will result in only $4.5 \%$ of the input power being dissapted in the circuit. It is very important to keep the dissipated power as low as possible. As the power losses increase, the power handling capacity of the components must be increased. This will lead to increased size and cost of the components. The example below illustrates how this value was obtained.

Given that $P_{I n}=10 \mathrm{dBm}$ and the insertion loss $\left(P_{\text {InsertionLoss }}\right)=0.2 \mathrm{~dB}$.

$$
\begin{gathered}
P_{\text {Out }}=P_{\text {In }}-P_{\text {InsertionLoss }}, \\
P_{\text {Out }}=10-0.2=9.8 \mathrm{dBm} .
\end{gathered}
$$

Converting $9.8 \mathrm{dBm}$ to $\mathrm{mW}$ yields

$$
P_{\text {Out }}=0.001\left(10^{\frac{9.8}{10}}\right)=9.55 \mathrm{~mW}
$$

The percentage of power dissipated in the circuit is

$$
\frac{10-9.55}{10} \times 100=4.5 \%
$$

\section{I.2. Desired Return Loss}

With a $20 \mathrm{~dB}$ return loss, 99\% of the source power is applied to the circuit. While making sure that all of the power is supplied to the circuit, this return loss is also important since the VSWR (1.2:1) will be low. High VSWR could apply very high voltages to the circuit thereby decreasing reliability. An example is used to 
illustrate how this value was obtained.

Given that $P_{I n}=10 \mathrm{dBm}$ and return loss $\left(P_{\text {ReturnLoss }}\right)=20 \mathrm{~dB}$. Then,

$$
P_{\text {Reflected }}=P_{I n}-P_{\text {ReturnLoss }},
$$

so that

$$
P_{\text {Reflected }}=10-20=-10 \mathrm{dBm},
$$

and converting $-10 \mathrm{dBm}$ into watts yields

$$
P_{\text {Reflected }}=0.001 \times 10^{\frac{-10}{10}}=0.1 \mathrm{~mW} .
$$

The percentage of power that is transmitted is

$$
\frac{P_{I n}-P_{\text {Reflected }}}{P_{\text {In }}} \times 100,
$$

or

$$
\frac{10-0.1}{10} \times 100=99 \% \text {. }
$$

\section{I.3. $920 \mathrm{MHz}$ Insertion Loss and Return Loss Analysis}

The insertion loss at $920 \mathrm{MHz}$ was $0.45 \mathrm{~dB}$ (the worst case). This would result in about $10 \%$ of the input power being dissipated in the circuit, a good result. The return loss at $920 \mathrm{MHz}$ exceeded the target value from 2 to $8 \mathrm{~dB}$, an excellent result.

\section{I.4. $2450 \mathrm{MHz}$ Insertion Loss and Return Loss Analysis}

The insertion loss at $2450 \mathrm{MHz}$ was $2 \mathrm{~dB}$. This was the worst case. This resulted in about $37 \%$ of the input power being dissipated, an acceptable result. The return loss was $8 \mathrm{~dB}$, which would result in approximately $16 \%$ of the input power being reflected, a fair result. 


\section{APPENDIX J. ANALYSIS OF THE MURATA ELECTRONICS CAPACITOR AND INDUCTOR MODELS USED IN THE ADS SIMULATION}

Capacitor and inductor models from Murata Electronics were used in the simulation reported in chapter 4 . To verify that the models were correct, ADS simulations were performed comparing the Murata models with the traditional lumped-element models for capacitors and inductors. For capacitors, the model includes the capacitor, the equivalent series inductance (ESL), the equivalent series resistance (ESR), and the capacitance between the printed circuit board mounting pads [21]. For inductors, the model includes the inductor, the series resistance and the capacitance between each winding of the inductor [22]. This capacitance is usually referred to as the interwinding capacitance. The Murata datasheets for the capacitor (GRM1555C1H200JZ01) and inductor (LQG15HH47NJ02) did not provide of this

information. Therefore estimated values were used that were based on the package size (JEDEC 0402). Refer to Tables $\mathrm{J}$ and $\mathrm{J}$ for the initial values for the capacitor and inductor models

The simulation schematics for the capacitor and inductor are shown in Figures J.1 and J.3 respectively. The circuit was optimized and the outputs are shown in Figures J.2 and J.4. From the results, the Murata Electronics model matches very closely to the standard capacitor and inductor models.

Table J.1. Capacitor Values.

\begin{tabular}{|c|c|}
\hline Murata Capacitor Value & $20 \mathrm{pF}$ \\
\hline Starting Capacitor Value & $20 \mathrm{pF}$ \\
\hline ESR & $0.5 \Omega$ \\
\hline ESL & $0.5 \mathrm{nH}$ \\
\hline PCB Pad Capacitance & $1 \mathrm{pF}$ \\
\hline
\end{tabular}




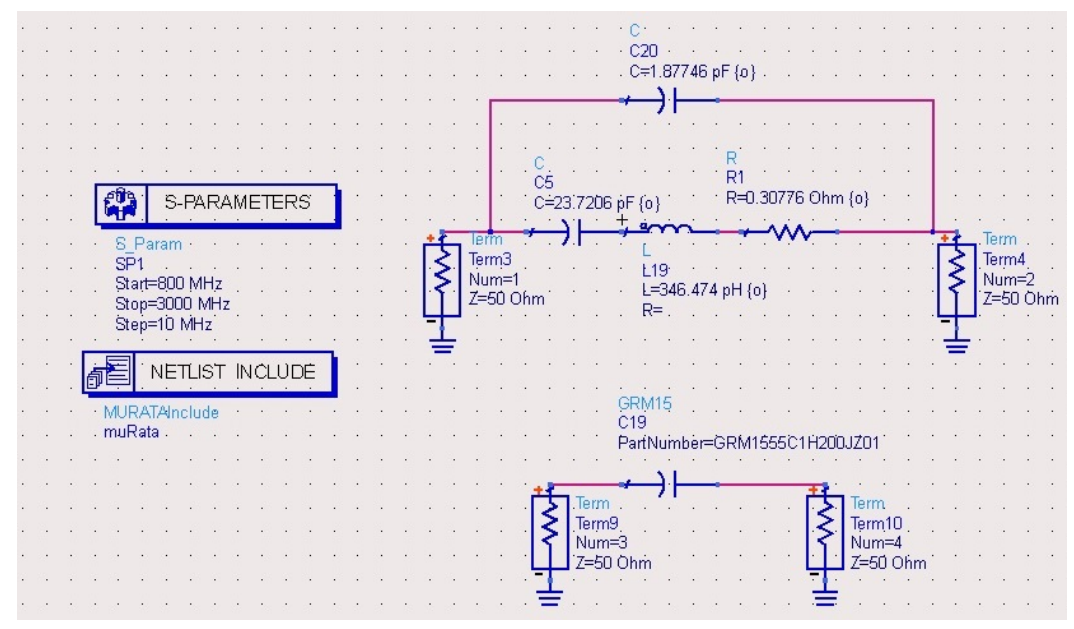

Figure J.1. Comparison between a Murata Electronics Capacitor and Ideal ADS Component Capacitor Model.
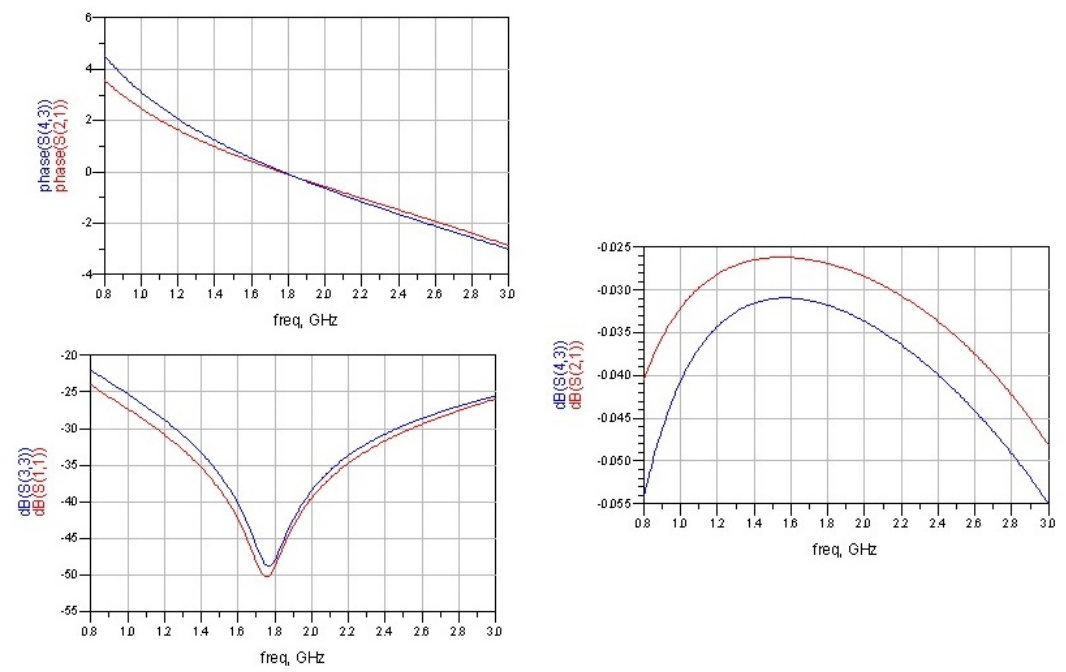

Figure J.2. Comparison between a Murata Electronics Capacitor and Ideal ADS Component Capacitor Model. 
Table J.2. Inductor Values.

\begin{tabular}{|c|c|}
\hline Murata Inductor Value & $47 \mathrm{nH}$ \\
\hline Starting Inductor Value & $47 \mathrm{nH}$ \\
\hline Series Resistance & $10 \Omega$ \\
\hline Interwinding Capacitance & $0.5 \mathrm{pF}$ \\
\hline
\end{tabular}

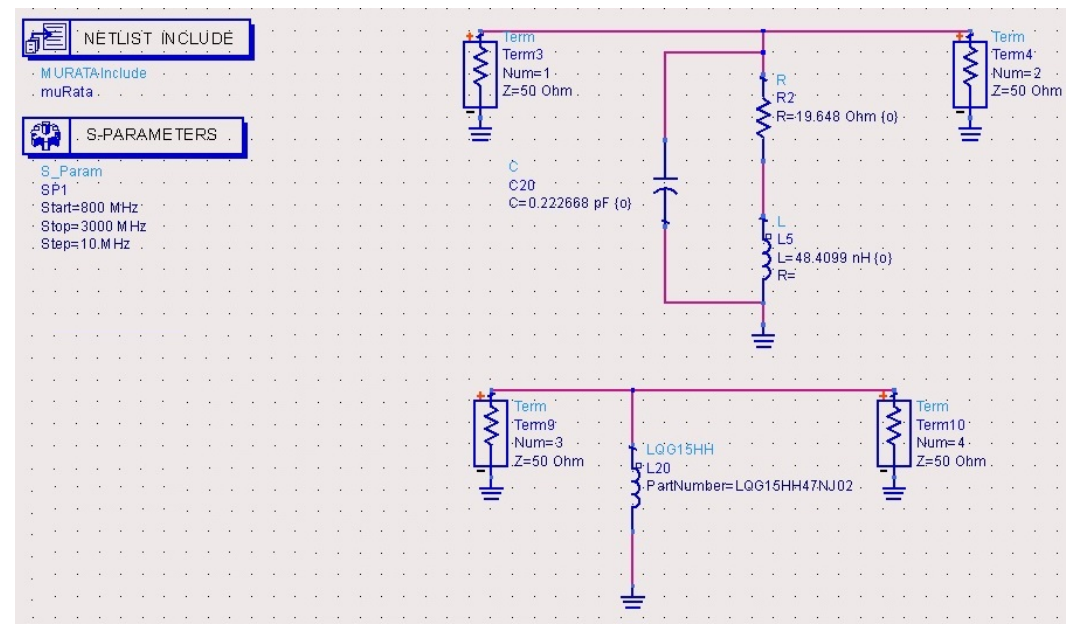

Figure J.3. Comparison between a Murata Electronics Inductor and Ideal ADS Component Inductor Model. 

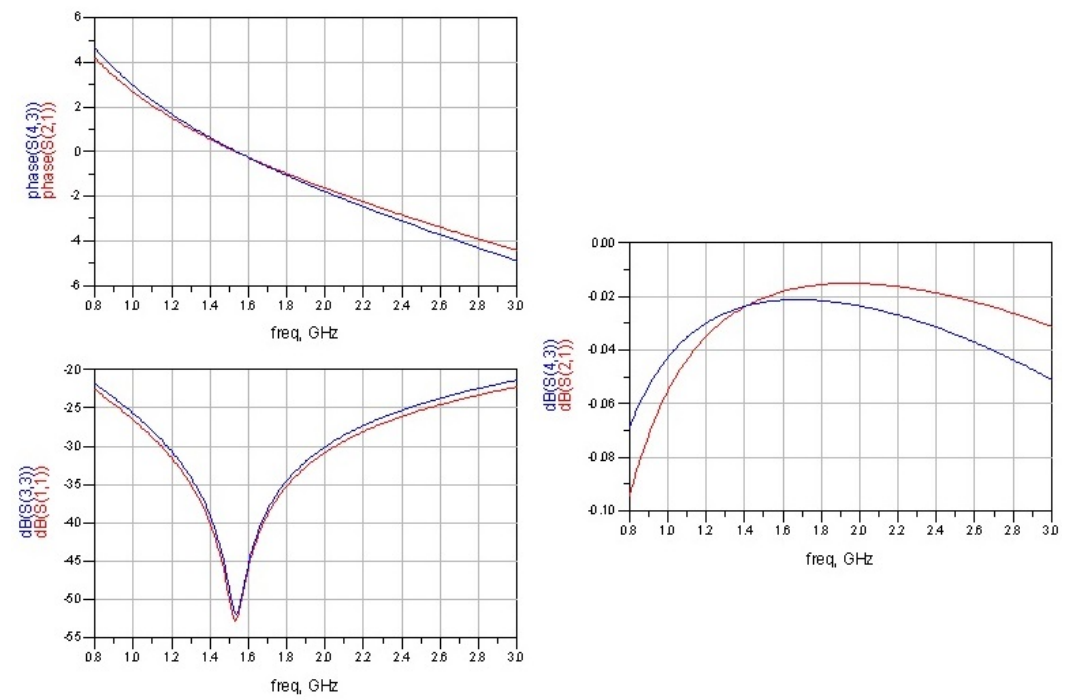

Figure J.4. Comparison between a Murata Electronics Inductor and Ideal ADS Component Inductor Model. 